MARCELO ABDALA DAHER

\title{
ARRANJOS DE MICROFONES PARA RECONSTRUÇÃO DE IMAGENS ACÚSTICAS
}




\title{
MARCELO ABDALA DAHER
}

\section{ARRANJOS DE MICROFONES PARA RECONSTRUÇÃO DE IMAGENS ACÚSTICAS}

\author{
Versão Corrigida \\ Dissertação apresentada à Escola Po- \\ litécnica da Universidade de São Paulo para \\ obtenção do Título de Mestre em Ciências.
}




\title{
MARCELO ABDALA DAHER
}

\section{ARRANJOS DE MICROFONES PARA RECONSTRUÇÃO DE IMAGENS ACÚSTICAS}

\author{
Versão Corrigida
}

Dissertação apresentada à Escola Politécnica da Universidade de São Paulo para obtenção do Título de Mestre em Ciências.

Área de Concentração:

Sistemas Eletrônicos

Orientador:

Prof. Dr. Vítor Heloiz Nascimento 
Este exemplar foi revisado e alterado em relação à versão original, sob responsabilidade única do autor e com a anuência de seu orientador. São Paulo, 30 de Novembro de 2020.

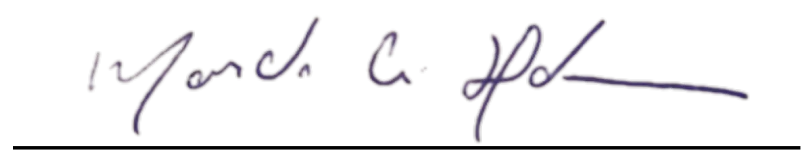

Marcelo Abdala Daher

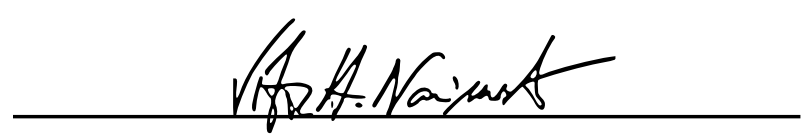

Prof. Dr. Vítor Heloiz Nascimento 
Autorizo a reprodução e divulgação total ou parcial deste trabalho, por qualquer meio convencional ou eletrônico, para fins de estudo e pesquisa, desde que citada a fonte.

Este exemplar foi revisado e corrigido em relação à versão original, sob responsabilidade única do autor e com a anuência de seu orientador.

São Paulo, 12 de Dezembro de 2020

Assinatura do autor:

Mand.

a. Pfo

Assinatura do orientador: IfotA. Nainat

\section{Catalogação-na-publicação}

\section{Daher, Marcelo}

Arranjos de microfones para reconstrução de imagens acústicas / M. Daher -- versão corr. -- São Paulo, 2020

$71 \mathrm{p}$.

Dissertação (Mestrado) - Escola Politécnica da Universidade de São Paulo. Departamento de Engenharia de Sistemas Eletrônicos.

1.Processamento digital de sinais 2.Acústica 3.Transformadas rápidas I.Universidade de São Paulo. Escola Politécnica. Departamento de

Engenharia de Sistemas Eletrônicos Il.t. 


\section{RESUMO}

Arranjos de microfones são ferramentas utilizadas em diversas aplicações, como estimação de direção de chegada (direction of arrival ou DOA), filtragem espacial, e estimação de imagens acústicas. Esse estudo tem em mente arranjos bidimensionais para tratamento de ondas se propagando no ar.

Muitos algoritmos para localização desenvolvidos com arranjos unidimensionais em mente não se traduzem bem para arranjos bidimensionais pelo custo computacional atrelado ao aumento quadrático do número de microfones. Para reduzir esse custo, exploramos uma classe de arranjos bidimensionais, denominada separáveis, que permite o uso da Kronecker array transform (KAT), transformada essa desenvolvida para reduzir a complexidade computacional de algoritmos para estimação de imagens acústicas. Recentemente a KAT foi estendida para acelerar algoritmos tradicionais (lineares) de filtragem espacial, mas a sua utilização com algoritmos de recontrução esparsa para filtragem espacial ainda não era possível.

Neste trabalho mostramos como utilizar a KAT em algoritmos de reconstrução esparsa para filtragem espacial. Estudamos também a precisão de algoritmos de recontrução esparsa em aplicações de estimação de DOA e recuperação de sinais provenientes de uma direção específica.

Para fins de qualificação de um arranjo, analisamos alguns parâmetros que nos permitem relacionar o posicionamento dos microfones com a verossimilhança da estimação de imagens acústicas ao se utilizar algoritmos de reconstrução esparsa, podendo ser utilizados como uma ferramenta de projeto para um novo arranjo. Também determinamos analiticamente a discretização do espaço que minimiza um destes parâmetros, a coerência.

Parte do trabalho envolveu o desenvolvimento de um software de simulação para campos acústicos incidentes em arranjos separáveis, onde comparamos algoritmos tradicionais de beamforming com algoritmos de reconstrução esparsa para localização de fontes e reconstrução de sinais no tempo. Os algoritmos que promovem esparsidade apresentaram maior precisão em estimação de DOA e grande melhora na recuperação direcional de sinais no tempo.

Palavras-Chave - Arranjos de microfones, beamforming, separação de fontes, redução de ruído, reconstrução esparsa. 


\section{ABSTRACT}

Microphone arrays are tools with various applications, such as direction of arrival (DOA) estimation, spatial filtering, and acoustic image estimation. This study takes in consideration two-dimensional arrays for waves traveling trough air.

Several algorithms developed with one-dimensional arrays in mind do not translate well to two-dimensional arrays, due to the increase in computational cost attached to the quadratic increase in the number of microphones. To reduce this computational cost for two-dimensional array processing, the Kronecker array transform (KAT) was introduced, which can be used when we have a separable array geometry. Recently, the KAT has been extended to accelerate traditional (linear) spatial filtering algorithms, but its use with sparse reconstruction algorithms was not yet possible.

This document shows how to implement the KAT to accelerate sparse reconstruction algorithms for spatial imaging applications. We also study the precision of sparse reconstruction algorithms in DOA estimation and directional signal recovery.

To qualify an array geometry, we study parameters that allow us to correlate microphone positions with the verisimilitude of an acoustic image generated by this array trough sparse techniques. Such parameter analysis can be useful when specifying the array geometry in the design stage. We also derive the space discretization that yields the optimum value of one of these parameters (coherence).

To test each algorithm, we developed a simulation software for an incident sound field on a separable array, where we compare traditional beamforming algorithms with sparse reconstruction algorithms for source localization and directional signal recovery. the sparsity-based approach shows better performance in DOA estimation and a great improvement in time-domain signal recovery.

Keywords - Microphone arrays, beamforming, source separation, signal denoising, compressed sensing. 


\section{SUMÁRIO}

1 Introdução $\quad 8$

2 Características acústicas $\quad 11$

2.1 Campo acústico e parametrização do espaço . . . . . . . . . . . . . . . . . 11

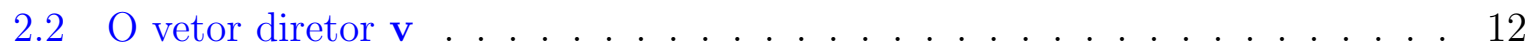

2.3 Considerações para arranjo separável . . . . . . . . . . . . . . . . . 14

$\begin{array}{lll}3 & \text { Imageamento Acústico } & 17\end{array}$

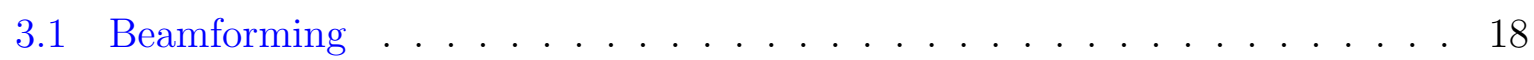

3.1.1 Beamformer Delay-And-Sum . . . . . . . . . . . . . . 19

3.1 .2 DAS-KAT . . . . . . . . . . . . . . . . . 19

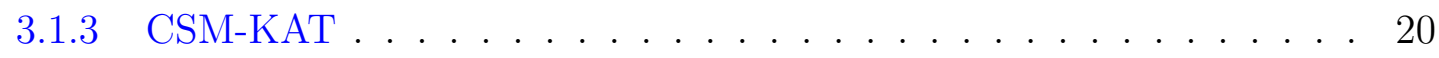

3.1.4 Beamformer MVDR . . . . . . . . . . . . . . . 22

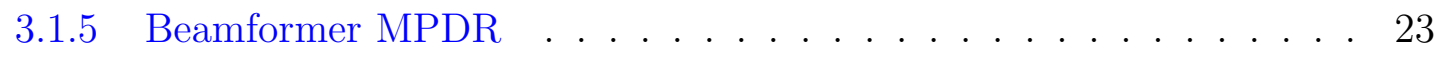

3.2 Deconvolução . . . . . . . . . . . . . . . . . . . . . 24

3.2 .1 Implementação por PSF . . . . . . . . . . . . . . . . . 24

3.2.1.1 Inicialização . . . . . . . . . . . . . . . . 27

3.2.1.2 Loop de iteração . . . . . . . . . . . . . . . . . . . 27

3.2 .2 Implementação por matriz A . . . . . . . . . . . . . . . 27

3.2 .2 .1 Inicialização . . . . . . . . . . . . . . . . . . . . 29

3.2.2.2 Loop de iteração . . . . . . . . . . . . . . . . . . . . . . 29

3.2 .3 Aceleração via KAT . . . . . . . . . . . . . . . . . . . . . . . . 30

3.2.3.1 Inicialização . . . . . . . . . . . . . . . . . . . . . . 31

3.2.3.2 Loop de iteração . . . . . . . . . . . . . . . . . . . . . . 31 
4.1 Algoritmos para reconstrução esparsa . . . . . . . . . . . . . . . . . . . 32

4.1.1 Algoritmos de otimização convexa . . . . . . . . . . . . . . . . . . 33

4.1 .2 Algoritmos gulosos . . . . . . . . . . . . . . . . . 33

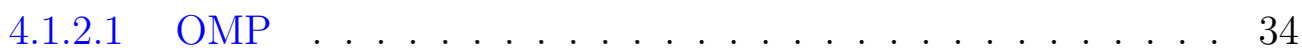

4.2 Parâmetros de reconstrução . . . . . . . . . . . . . . . . . . . 35

$4.2 .1 \quad$ Spark . . . . . . . . . . . . . . . . . . 35

4.2 .2 Coerência . . . . . . . . . . . . . . . . 36

5 Discretização do espaço $\quad 38$

6 Resultados numéricos $\quad 41$

6.1 Simulador de Imageamento Acústico . . . . . . . . . . . . . . . . . . . . . 41

6.1.1 Estrutura do simulador . . . . . . . . . . . . . . . . . . . 42

6.1.1.1 FarFieldSignal . . . . . . . . . . . . . . . . . 42

6.1.1.2 GridArray ................... . . 43

6.1.1.3 Imager . . . . . . . . . . . . . . . . . . . . 44

6.1.1.4 SignalEstimator . . . . . . . . . . . . . . . 46

6.2 Caracterização do sistema . . . . . . . . . . . . . . . . . . . . 47

6.2.1 Geometria ........................ 47

6.2 .2 Resolução . . . . . . . . . . . . . . . . . . . . . . . . . . . . . 49

6.3 Imageamento e DOA . . . . . . . . . . . . . . . . . . 51

6.4 Reconstrução do sinal no tempo . . . . . . . . . . . . . . . . . 56

$\begin{array}{lll}7 & \text { Conclusão } & 60\end{array}$

$\begin{array}{ll}\text { Referências } & 62\end{array}$

Anexo A - Publicações relevantes $\quad 64$ 


\section{INTRODUÇÃO}

Esse documento trata sobre o processamento de sinais para arranjos de microfones com finalidade de imageamento acústico.

Uma imagem acústica é uma representação do mapa de intensidades de uma faixa de frequências de um campo acústico observado em um ponto referencial. A posição de cada pixel dessa imagem representa uma direção observada para a chegada de um sinal, e o valor desse pixel representa a intensidade estimada desse sinal. Cobrimos aqui algoritmos que estimam não somente a distribuição de fontes acústicas, mas a intensidade do sinal proveniente de cada uma das fontes e ainda que reconstroem no domínio do tempo os sinais emitidos por elas.

O primeiro algoritmo desenvolvido com esse intuito foi o beamformer convencional ou delay-and-sum (DAS), um método de imageamento computacionalmente simples, mas que apresenta grandes lóbulos laterais que afetam a imagem gerada. A presença de lóbulos reduz a precisão na localização de fontes e gera artefatos em outras direções da imagem. Os beamformers MVDR e MPDR [1] aprimoram o isolamento da fonte a ser localizada em relação a ruído, mas exigem inúmeras medições para estimação de uma matriz de autocorrelação do ruído, no caso do MVDR, e do sinal, no caso do MPDR. Distorções causadas por lóbulos são reduzidas mas ainda ocorrem. Técnicas de deconvolução, como o estado da arte DAMAS2 [2], modelam o efeito de lóbulos a uma convolução da imagem ideal a uma resposta ao impulso, e tentam deconvoluir a imagem. O efeito dos lóbulos são fortemente mitigados, mas suas técnicas iterativas são computacionalmente custosas e descartam informação de fase do sinal, não permitindo a recuperação direcional de um sinal no tempo, além de partir do pressuposto de que os sinais incidentes são descorrelacionados, aproximação nem sempre verdadeira. Similarmente, técnicas de covariance fitting [3] melhoram a precisão das imagens a partir de uma minimização convexa utilizando a matriz de covariância das medições, mas também apresentam desvantagens ao exigir múltiplas medições para estimação da matriz de covariância, e por não permitirem a recuperação de um sinal no tempo. 
Neste trabalho exploramos o uso da Kronecker array transform (KAT), aplicável a arranjos de geometria separável (seção 2.3). A KAT [4] é uma transformada desenvolvida para reduzir a complexidade computacional de algoritmos para estimação de imagens acústicas, como o DAS, DAMAS2 e covariance-fitting, e que foi recentemente estendida para acelerar algoritmos tradicionais (lineares) de filtragem espacial. Mostramos como utilizar a KAT para filtragem espacial com algoritmos de reconstrução esparsa, e indicamos como otimizar a geometria de um arranjo, dado o número de microfones, para obter uma maior resolução no espaço.

A aplicação de reconstrução esparsa para beamforming foi proposta previamente, e Xenaki et al. [5] estudou a performance de algoritmos baseados em reconstrução esparsa para estimação de direção de chegada (direction of arrival, ou DOA), considerando arranjos unidimensionais aleatórios, e relacionando parâmetros de performance, como resolução, à coerência da matriz de medidas. Nossa análise também é baseada na medida de coerência, estendendo seus resultados em 4 maneiras:

i: consideramos arranjos bidimensionais;

ii: consideramos arranjos separáveis, aos quais a KAT pode ser aplicada;

iii: avaliamos a performance de um arranjo em função da frequência;

iv: avaliamos a recuperação de sinais no tempo.

Outra contribuição é nossa análise sobre a discretização do espaço para minimizar a coerência, uma vez que utilizamos um número finito de direções fixas. Além disso, apesar de existirem algoritmos para localização de fonte que levam em consideração todo o espectro de um sinal de banda larga, mostramos que, para um número pequeno de fontes, o processamento independente de cada frequência já nos traz um bom resultado para a recuperação de um sinal no tempo vindo de uma direção específica.

Da teoria de reconstrução esparsa, buscamos parâmetros como spark e coerência para quantificar para esse sistema a qualidade possível de se atingir com a reconstrução. Utilizamos esses parâmetros para comparar o desempenho esperado entre arranjos com diferentes geometrias de microfones, visando definir uma geometria adequada ao iniciar o projeto de um novo arranjo.

Parte do trabalho envolveu o desenvolvimento de um simulador para campos acústicos incidentes em arranjos separáveis para testar os algoritmos pesquisados, e esse simulador é a origem dos resultados numéricos apresentados. Como os algoritmos implementados 
tratam os sinais captados pelo arranjo de microfone no domínio da frequência, o software desenvolvido interpola no domínio do tempo o campo acústico para criar os sinais capturados pelo arranjo de microfones, o que nos permite validar o modelo do sistema. $\mathrm{O}$ simulador foi publicado sob a licença MIT em [6].

A organização do texto é a seguinte. O capítulo 2 descreve a modelagem do sistema no espaço e define a notação utilizada. O capítulo 3 introduz algoritmos de imageamento e explica a necessidade de deconvolução de imagens obtidas através de beamforming. $\mathrm{O}$ capítulo 4 explica o problema de sistemas lineares sub-determinados e introduz como podemos encontrar uma solução para o sistema dada informação a priori sobre a esparsidade do sinal em algum domínio, não necessariamente o domínio do tempo. Cobre também alguns parâmetros do sistema que podem trazer garantias sobre a unicidade da solução. O capítulo 5 demonstra como pode ser feita de forma ótima a seleção das direções de interesse e a discretização do espaço para a utilização de técnicas de reconstrução esparsa. O capítulo 6 detalha o trabalho realizado computacionalmente, começando pelo simulador de arranjo acústico desenvolvido para testar os algoritmos aqui descritos. Analisamos os parâmetros de reconstrução de diferentes geometrias de arranjos, realizamos testes de precisão em relação à direção de chegada para diferentes algoritmos e comparamos entre esses algoritmos a precisão da reconstrução de sinais no tempo para uma direção específica. Finalmente o capítulo 7 apresenta conclusões e sugestões para possíveis trabalhos futuros.

Parte do trabalho apresentado nessa dissertação foi publicado em [7], onde qualificamos objetivamente geometrias de arranjos sob o ponto de vista da reconstrução esparsa, e sugerimos a aceleração do cálculo da coerência de sistemas separáveis através de manipulações do produto de Kronecker. Além disso, estudamos para sistemas separáveis a qualidade dos resultados provenientes de algoritmos de reconstrução esparsa na estimação de DOA para fontes de campo distante, e na recuperação de um sinal no tempo proveniente de uma direção específica. O artigo também analisa sob o ponto de vista de reconstrução esparsa a discretização ótima do espaço detalhada no capítulo 5 e a influência da frequência de operação e resolução do espaço na qualidade estimada de reconstrução. 


\section{CARACTERÍSTICAS ACÚSTICAS}

Aqui entraremos em detalhe sobre as propriedades acústicas do sistema, as aproximações feitas e o modelo utilizado.

\subsection{Campo acústico e parametrização do espaço}

Arranjos de microfones permitem separar sinais incidindo sobre o arranjo de direções diferentes devido às diferenças de atraso, e em menor grau de ganho, obtidas para cada direção de propagação. Neste trabalho, as fontes são consideradas distantes o suficiente para que a onda incidente de cada direção seja considerada plana, e o campo distante.

Para fins de reconstrução, o campo distante é discretizado em $N_{\operatorname{DirX}} \times N_{\operatorname{Dir} Y}=N_{\operatorname{Dir}}$ direções, e cada direção é descrita por um vetor direcional unitário u, normal a uma onda plana.

A representação do vetor $\mathbf{u}$ é derivada de coordenadas esféricas onde $\theta$ e $\phi$ são respectivamente os ângulos polar e azimutal, da seguinte forma (ver figura 1):

$$
\mathbf{u}=\left[\begin{array}{c}
u_{x} \\
u_{y} \\
\sqrt{1-u_{x}^{2}-u_{y}^{2}}
\end{array}\right]=\left[\begin{array}{c}
\sin \theta \cos \phi \\
\sin \theta \sin \phi \\
\cos \theta
\end{array}\right] .
$$

Como os arranjos de microfones que trataremos aqui são planos e separáveis, o sistema de coordenadas será posicionado com sua origem no centro do arranjo e com o plano $x y(z=0)$ no plano do arranjo para facilitar a representação.

O sinal de campo distante será representado em uma imagem acústica com cada pixel representando uma direção e tendo um vetor $\mathbf{u}$ atribuído a si. A magnitude desse pixel é proporcional à intensidade do sinal vindo da direção representada. As direções discretas são distribuídas em $[-1,1]$ para $u_{x}$ e $u_{y}$ da equação (2.1), determinando assim o vetor $\mathbf{u}$ atribuído a cada pixel. Repare que, uma vez que as coordenadas do vetor u só podem ser 


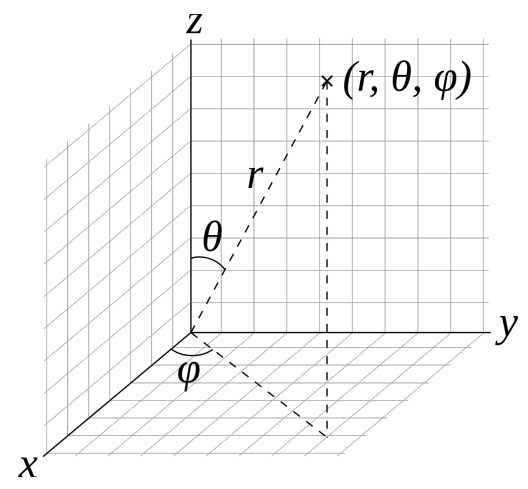

Figura 1: Sistema de coordenadas esféricas.

números reais, os pixels que contém informação válida estão contidos dentro do círculo unitário.

\subsection{O vetor diretor $\mathrm{v}$}

Para um arranjo com $N_{M i c X} \times N_{M i c Y}=N_{M i c}$ microfones, seja $\mathbf{p}_{i}=\left[p_{x}, p_{y}, p_{z}\right]^{T}$ a posição no espaço $x y z$ do i-ésimo microfone.

Utilizaremos para o processamento do sinal a diferença de tempo entre a chegada de um sinal na origem do sistema de coordenadas e no microfone posicionado em $\mathbf{p}_{i}$, para uma onda plana vinda de uma direção $\mathbf{u}$, isto é, propagando-se na direção $-\mathbf{u}$, conforme mostra a figura 2. Esse atraso será igual à diferença de distância $\Delta r$ percorrida dividida pela velocidade de propagação da onda, e no espaço euclidiano a diferença de distância percorrida pode ser calculada como a projeção de $\mathbf{p}_{i}$ em $\mathbf{u}$, ou seu produto escalar.

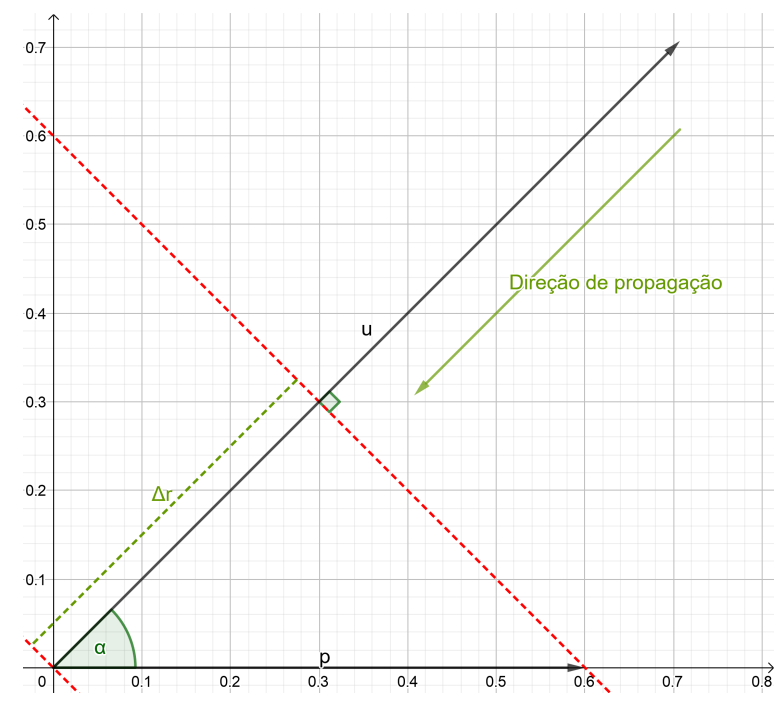

Figura 2: Vetores $\mathbf{u}$ e $\mathbf{p}$, e a diferença de distância $\Delta r$ percorrida por uma frente de onda plana para chegar a um microfone em $\mathbf{p}$ e outro na origem. 


$$
\Delta r=\|\mathbf{p}\| \cos \alpha=\frac{\mathbf{u}^{T} \mathbf{p}}{\|\mathbf{u}\|}=\mathbf{u}^{T} \mathbf{p}
$$

O atraso $\tau_{i}$ em relação à origem com que incide uma onda plana vinda da direção $\mathbf{u}$, em um microfone $i$ pode ser calculado a partir de $\Delta r$ por

$$
\tau_{i}=-\frac{\mathbf{u}^{T} \mathbf{p}_{i}}{c}
$$

onde $c$ é a constante da velocidade de propagação da onda no meio. No caso de imagens acústicas consideramos a velocidade de propagação do som no ar.

Os sinais adquiridos serão tratados na frequência, onde o atraso equivalerá a uma diferença de fase. No caso de ondas esféricas seria necessário considerar também a diferença de amplitude.

Ao processar os sinais na frequência, temos a vantagem de poder calcular o sinal com atraso através de uma multiplicação a partir do sinal discreto original. Se estivéssemos tratando o sinal no tempo, teríamos que realizar a interpolação do sinal para calcular seu valor, exceto para raros casos em que o atraso é múltiplo do período de amostragem.

Note que a implementação no domínio da frequência usando a FFT implica aproximar o sinal observado na $k$-ésima raia da FFT como correspondente unicamente à frequência $\omega_{k}=k 2 \pi / N$, em que $N$ é o número de pontos da FFT. No entanto, sabe-se que o sinal observado em cada raia da FFT corresponde à saída de um filtro passa-banda centrado em $\omega_{k}$, com ganho não desprezível mesmo para frequências relativamente afastadas de $\omega_{k}$. Como isto implica uma aproximação ao se fazer o processamento no domínio da frequência, no capítulo 6 usamos interpolação no tempo para gerar aproximações mais precisas dos sinais observados em cada microfone, de maneira que o simulador também leve em conta as aproximações feitas ao se fazer processamento no domínio da frequência. Veja a discussão após a equação (2.5).

Para cada frequência $\omega$ e direção incidente $\mathbf{u}$, podemos então definir um vetor-coluna $\mathbf{v}$ contendo as defasagens para cada microfone que chamaremos de vetor diretor:

$$
\mathbf{v}(\omega: \mathbf{u})=\left[\begin{array}{c}
e^{j \omega \tau_{1}} \\
e^{j \omega \tau_{2}} \\
\vdots \\
e^{j \omega \tau_{\left(N_{M i c}\right)}}
\end{array}\right]=\left[\begin{array}{c}
e^{-j \omega \mathbf{u}^{T} \mathbf{p}_{1} / c} \\
e^{-j \omega \mathbf{u}^{T} \mathbf{p}_{2} / c} \\
\vdots \\
e^{-j \omega \mathbf{u}^{T} \mathbf{p}_{\left(N_{M i c}\right)} / c}
\end{array}\right]
$$

Agrupando os vetores $\mathbf{v}(\mathbf{u})$ para as direções observadas, definimos a matriz $\mathbf{V}(\omega)$ com 
$N_{M i c}$ linhas e $N_{\text {Dir }}$ colunas:

$$
\mathbf{V}(\omega)=\left[\begin{array}{l:l:l:l}
\mathbf{v}\left(\omega: \mathbf{u}_{1}\right) & \mathbf{v}\left(\omega: \mathbf{u}_{2}\right) & \cdots & \mathbf{v}\left(\omega: \mathbf{u}_{\left(N_{\text {Dir }}\right)}\right)
\end{array}\right]
$$

Definimos o vetor $\mathbf{x}(\omega)=\left[x_{1}(\omega), x_{2}(\omega), \ldots, x_{N m i c}(\omega)\right]^{T}$ que contém a FFT do sinal de todos os microfones para a faixa de frequência centrada em $\omega$ e o vetor $\mathbf{y}(\omega)=$ $\left[y_{1}(\omega), y_{2}(\omega), \ldots, y_{N d i r}(\omega)\right]^{T}$ contendo o sinal de campo distante. O sinal representado no domínio da frequência está sujeito a aproximações feitas ao se usar a FFT, como vazamento espectral e distorções de amplitude para frequências situadas nas extremidades das bandas. Precisamos ter consciência desses efeitos ao processar um único bin. Os efeitos de espalhamento podem ser mitigados ao se processar o sinal em banda larga, por exemplo utilizando para imageamento ou estimação de DOA a média da potência estimada para vários bins.

O vetor de sinais $\mathbf{x}(\omega)$ que atinge o arranjo de microfones será a superposição dos sinais provenientes de cada direção. O vetor $\mathbf{x}(\omega)$ é calculado nesse modelo por:

$$
\mathbf{x}(\omega)=\mathbf{V}(\omega) \mathbf{y}(\omega)
$$

Como o processamento no domínio da frequência modela o sinal observado na $k$-ésima raia da FFT, a equação (2.6) é na verdade uma aproximação, pois $x\left(\omega_{k}\right)$ contém componentes de outras frequências, devido à operação de filtragem correspondente à FFT.

\subsection{Considerações para arranjo separável}

Em um arranjo separável os microfones estão sobre um reticulado, ou seja, nas posições $(x, y)=\left(x_{i}, y_{j}\right)$, em que $i=1 \cdots N_{D i r X}, j=1 \cdots N_{D i r Y}$. Um exemplo de arranjo separável está na figura 3 , onde cada círculo vermelho representa um sensor.

Nossa parametrização do espaço (escolha dos vetores u) também é separável. Em geral, utilizaremos $u_{x}$ e $u_{y}$ uniformemente distribuídos em $[-1,1]$.

O produto de Kronecker entre uma matriz $\mathbf{A}_{m \times n}$ e uma matriz $\mathbf{B}_{p \times q}$ resulta em uma matriz $\mathbf{C}_{m p \times n q}$ da forma

$$
\mathbf{A}_{m \times n} \otimes \mathbf{B}_{p \times q}=\left[\begin{array}{ccc}
a_{1,1} \mathbf{B} & \cdots & a_{1, n} \mathbf{B} \\
\vdots & \ddots & \vdots \\
a_{m, 1} \mathbf{B} & \cdots & a_{m, n} \mathbf{B}
\end{array}\right]_{m p \times n q}
$$




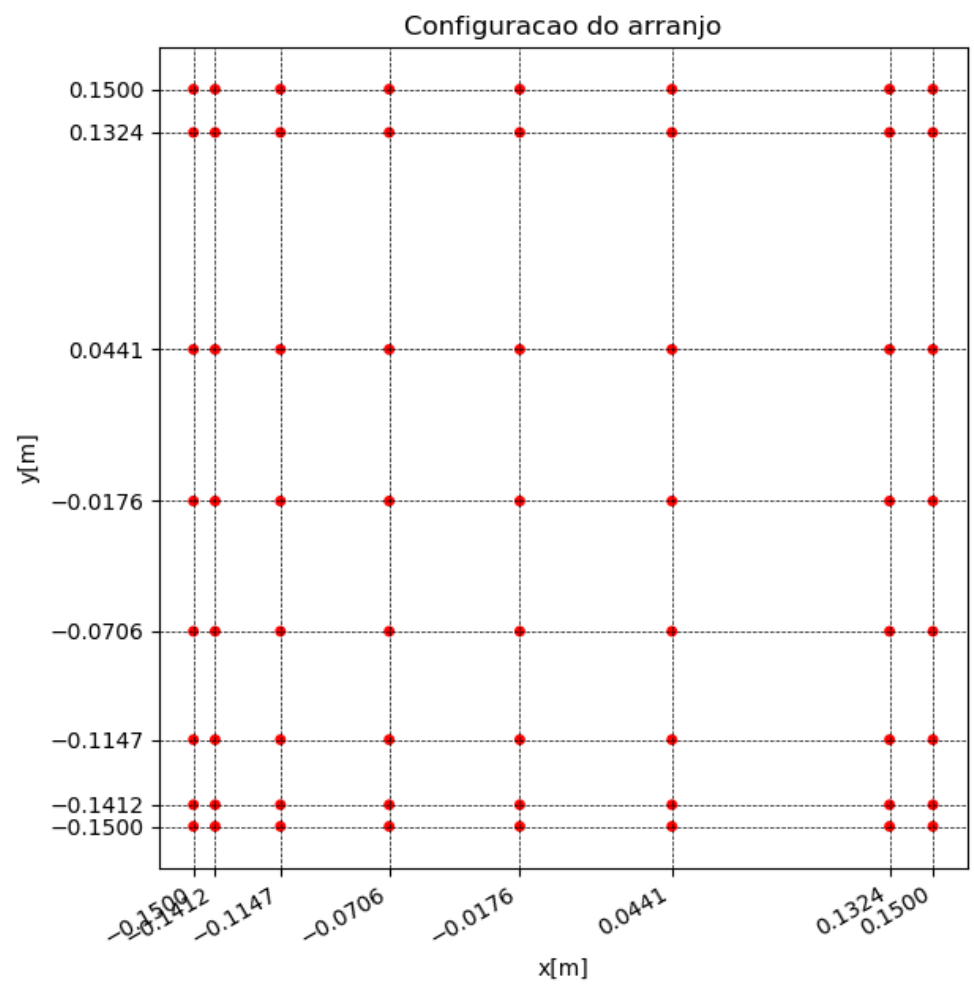

Figura 3: Exemplo de arranjo separável.

A matriz $\mathbf{C}$ possui elementos $a_{i, j} b_{k, l}$, para qualquer $i, j, k, l$ válido.

Com o arranjo e as direções sendo separáveis, a matriz $\mathbf{V}$ de dimensão $\left[N_{M i c} \times N_{D i r}\right]$, onde $N_{M i c}=N_{M i c X} N_{M i c Y}$ e $N_{D i r}=N_{D i r X} N_{D i r Y}$, pode ser decomposta no produto de Kronecker [8]

$$
\mathbf{V}=\mathbf{V}_{x} \otimes \mathbf{V}_{y}
$$

onde $\mathbf{V}_{x}$ é uma matriz de dimensão $\left[N_{M i c X} \times N_{D i r X}\right]$ e $\mathbf{V}_{y}$ uma matriz de dimensão $\left[N_{M i c Y} \times N_{D i r Y}\right]$, como mostrado a seguir.

Decompondo o produto entre os vetores $\mathbf{u}$ e $\mathbf{p}$ da equação (2.3), para um microfone qualquer no grid posicionado em $\mathbf{p}=\left[x_{i}, y_{j}, 0\right]$, chegamos a:

$$
\tau=-\frac{\left[u_{x}, u_{y}, \sqrt{1-u_{x}^{2}-u_{y}^{2}}\right]\left[x_{i}, y_{j}, 0\right]^{T}}{c}=-\frac{u_{x} x_{i}+u_{y} y_{j}}{c},
$$

logo cada elemento do vetor $\mathbf{v}(\omega: \mathbf{u})$ pode ser representado por:

$$
e^{j \omega \tau}=e^{-j \omega u_{x} x_{i} / c} e^{-j \omega u_{y} y_{j} / c} .
$$


Definindo $\mathbf{V}_{x}$ e $\mathbf{V}_{y}$ da seguinte maneira

$$
\begin{aligned}
\mathbf{V}_{x}= & {\left[\begin{array}{ccc}
e^{-j \omega(-1) x_{1} / c} & \ldots & e^{-j \omega(1) x_{1} / c} \\
e^{-j \omega(-1) x_{2} / c} & \ldots & e^{-j \omega(1) x_{2} / c} \\
\vdots & \ddots & \vdots \\
e^{-j \omega(-1) x_{\left(N_{M i c X}\right)} / c} & \ldots & e^{-j \omega(1) x_{\left(N_{M i c X}\right)} / c}
\end{array}\right]_{N_{M i c X} \times N_{D i r X}} } \\
\mathbf{V}_{y}= & {\left[\begin{array}{ccc}
e^{-j \omega(-1) y_{1} / c} & \ldots & e^{-j \omega(1) y_{1} / c} \\
e^{-j \omega(-1) y_{2} / c} & \ldots & e^{-j \omega(1) y_{2} / c} \\
\vdots & \ddots & \vdots \\
e^{-j \omega(-1) y_{\left(N_{M i c Y}\right)} / c} & \ldots & e^{-j \omega(1) y_{\left(N_{M i c Y}\right)} / c}
\end{array}\right]_{N_{M i c Y} \times N_{D i r Y}} }
\end{aligned}
$$

podemos escrever a matriz $\mathbf{V}$ segundo a equação (2.8).

Essa decomposição da matriz $\mathbf{V}$ nos permite acelerar o processamento dos dados explorando o produto de Kronecker [9]. Usaremos a propriedade

$$
\left(\mathbf{A}^{T} \otimes \mathbf{B}\right) \operatorname{vec}\{\mathbf{C}\}=\operatorname{vec}\{\mathbf{B C A}\}
$$

válida sempre que produto BCA for definido.

Como será visto na seção 3.2.3, para a estimação de imagens acústicas usam-se produtos do tipo $\mathbf{x}=\mathbf{V y}$, em que $\mathbf{x} \in \mathbb{C}^{N_{M i c}}, \mathbf{y} \in \mathbb{C}^{N_{\text {Dir }}}$ e $\mathbf{V} \in \mathbb{C}^{N_{M i c} \times N_{\text {Dir }}}$. O custo computacional de um produto deste tipo implementado diretamente é de $4 N_{M i c} N_{D i r}$ multiplicações reais. Por outro lado, usando (2.13) teremos $\mathbf{x}=\left(\mathbf{V}_{x} \otimes \mathbf{V}_{y}\right) \mathbf{y} \Rightarrow \mathbf{X}=$ $\mathbf{V}_{x} \mathbf{Y} \mathbf{V}_{y}^{T}$, em que $\mathbf{X} \in \mathbb{C}^{N_{M i c X} \times N_{M i c Y}}, \mathbf{Y} \in \mathbb{C}^{N_{D i r X} \times N_{D i r Y}}, \mathbf{V}_{x} \in \mathbb{C}^{N_{M i c X} \times N_{D i r X}}$ e $\mathbf{V}_{y} \in$ $\mathbb{C}^{N_{M i c Y} \times N_{D i r Y}}$, o que necessita do mínimo entre $\left(N_{M i c X} N_{M i c Y} N_{D i r X}+N_{\operatorname{Dir} X} N_{\operatorname{Dir} Y} N_{M i c Y}\right)$ e $\left(N_{M i c X} N_{M i c Y} N_{D i r Y}+N_{D i r X} N_{D i r Y} N_{M i c X}\right)$ operações. Para um arranjo de $8 \times 8$ microfones reconstruindo $32 \times 32$ direções, o número de operações é reduzido de $2^{18}$ para aproximadamente $2^{13}$. 


\section{IMAGEAMENTO ACÚSTICO}

Utilizaremos a seguinte representação ao tratar de imagens acústicas:

- $\mathbf{Y}(\omega)$ ou Y é uma matriz de dimensão $N_{\operatorname{Dir} X} \times N_{\operatorname{Dir} Y}$ que contém o coeficiente para a frequência $\omega$ da transformada de Fourier de tempo discreto (DTFT) do sinal originado de cada direção do espaço discreto;

- $\mathbf{y}(\omega)$ ou y é um vetor coluna de dimensão $N_{D i r} \times 1$, possuindo os mesmos elementos de $\mathbf{Y}(\omega)$ rearranjados em um vetor. Denotamos que $\mathbf{y}=\operatorname{vec}\{\mathbf{Y}\}$;

- $\mathscr{Y}(\omega)$ ou $\mathscr{Y}$ é a nossa imagem acústica, uma matriz de dimensão $N_{\operatorname{Dir} X} \times N_{\operatorname{DirY}}$ que contém a intensidade do sinal emitido para cada direção do espaço discreto, referente à baia $\omega$ da DTFT, e é dado por $\mathscr{Y}=\mathbb{E}\left\{|\mathbf{Y}|^{2}\right\}$;

- $y(\omega)$ ou y é um vetor coluna de dimensão $N_{D i r} \times 1$, possuindo os mesmos elementos de $\mathcal{Y}(\omega)$ rearranjados em um vetor, e é dado por $y=\mathbb{E}\left\{|\mathbf{y}|^{2}\right\}$. Denotamos que $y=\operatorname{vec}\{\mathscr{y}\}$. Essa é a nossa matriz de imagem acústica;

- O operador $\hat{-}$ denota uma estimativa. $\hat{x}$ é uma estimativa para $x$.

Uma imagem acústica mapeia intensidades de fontes sonoras em uma representação de direções no espaço. Através do imageamento acústico, podemos verificar a presença de fontes sonoras e suas direções de origem em relação ao arranjo de microfones. Essas imagens normalmente estão associadas à detecção e caracterização de fontes sonoras, além de apresentar aplicações em estudos de aerodinâmica e em análises de estruturas como salas de concerto e turbinas. Existem vários métodos para estimar uma imagem acústica, como métodos clássicos [1] de filtragem espacial utilizando beamformers, métodos de deconvolução [2] para aprimorar resultados iniciais visando eliminar efeitos de lóbulos laterais, além de técnicas de regularização que podem ser aplicadas às medidas dos microfones $[3,10]$.

Trabalharemos no domínio da frequência para todos os casos, estimando uma imagem acústica $\hat{\mathscr{Y}}(\omega)$ para uma certa banda. Para simplificar a notação, o índice $\omega$ será omitido. 
Alguns algoritmos, como o beamforming a seguir, estimam o sinal de campo distante $\hat{\mathbf{Y}}$, enquanto outros trabalham diretamente com a estimativa da intensidade do sinal $\hat{\mathscr{Y}}$. Neste capítulo apresentaremos alguns algoritmos que nos permitem utilizar a Kronecker array transform (KAT), e como implementá-la para reduzir custos computacionais de algoritmos de beamforming e deconvolução, dado que o algoritmo seja compatível e o sistema separável.

\subsection{Beamforming}

Um arranjo amostra espacialmente o campo sonoro nas posições dos microfones $\mathbf{p}_{n}$, $n=1 \cdots N_{M i c}$. O sinal de cada microfone é então processado por um filtro linear invariante no tempo, e a soma das saídas dos filtros resulta na nossa estimativa para a direção u.

No domínio da frequência, podemos representar a operação de convolução no tempo realizada pelos filtros por uma multiplicação. Olhando para uma única frequência, podemos denotar matricialmente nossa estimativa do sinal para a direção u (a soma da saída dos filtros aplicados ao sinal de cada microfone), por

$$
\hat{y}(\omega, \mathbf{u})=\mathbf{w}^{H}(\omega, \mathbf{u}) \mathbf{x}(\omega)
$$

onde w é um vetor coluna composto por $N_{\text {Mic }}$ pesos complexos, representando para essa frequência a operação linear realizada pelo banco de filtros.

A imagem acústica tem como objetivo representar a intensidade das fontes sonoras do campo que incide no arranjo. Sendo $y(\omega, \mathbf{u})$ a componente de frequência $\omega$ do sinal incidente da direção $\mathbf{u}$, a intensidade que cada pixel de $\boldsymbol{y}$ e $\mathcal{Y}$, dada sua direção u, visa representar é $E\left\{|y(\mathbf{u})|^{2}\right\}$, ou, em outras palavras, cada elemento de $\mathcal{y}$ e $\mathscr{Y}$ corresponde a $\mathbb{E}\left\{|\mathbf{y}(\mathbf{u})|^{2}\right\}$ para uma dada direção $\mathbf{u}$.

Obtendo os vetores $\mathbf{w}(\mathbf{u})$ (omitindo $\omega$ na notação) para todas as direções $\mathbf{u}$ de interesse, podemos compor a matriz $\mathbf{W}$ da forma

$$
\mathbf{W}=\left[\begin{array}{l:l:l:l}
\mathbf{w}\left(\mathbf{u}_{0}\right) & \mathbf{w}\left(\mathbf{u}_{1}\right) & \cdots & \mathbf{w}\left(\mathbf{u}_{\left(N_{D i r}-1\right)}\right)
\end{array}\right]
$$

e denotar vetorialmente a estimativa do sinal $\hat{\mathbf{y}}$

$$
\hat{\mathbf{y}}=\mathbf{W}^{H} \mathbf{x}
$$


Para obter a imagem acústica, precisamos estimar a intensidade das fontes, sendo feito a partir da estimativa do sinal

$$
\hat{y}=\mathbb{E}\left\{|\hat{\mathbf{y}}|^{2}\right\}
$$

sendo ||$^{2}$ o quadrado do módulo de um vetor, termo a termo. O valor esperado normalmente é estimado a partir da média de várias janelas [11].

\subsubsection{Beamformer Delay-And-Sum}

Do ponto de vista prático, o beamformer Delay-And-Sum (DAS) desloca no tempo os sinais de cada microfone para compensar o atraso de uma onda plana vinda da direção $\mathbf{u}$ em relação à origem do sistema de coordenadas e os soma, maximizando a potência para a direção de interesse [1]. O fator $1 / N_{\text {Mic }}$ compensa o ganho da soma dos sinais de $N_{\text {Mic }}$ microfones. O beamformer DAS é definido como a média da soma das saídas de cada microfone, atrasados de modo a alinhar os sinais chegando da direção u, ou seja:

$$
\mathbf{w}_{D A S}(\mathbf{u})=\frac{1}{N_{M i c}} \mathbf{v}(\mathbf{u})=\frac{\mathbf{v}(\mathbf{u})}{\mathbf{v}^{H}(\mathbf{u}) \mathbf{v}(\mathbf{u})}
$$

Uma vez tendo em mãos a matriz $\mathbf{V}$ que contém os vetores diretores, a matriz $\mathbf{W}_{D A S}$ pode ser obtida facilmente por

$$
\mathbf{W}_{D A S}=\frac{1}{N_{M i c}} \mathbf{V}
$$

Semelhante ao DAS, o beamformer Bartlett $\mathbf{w}_{\text {bartlett }}(\mathbf{u})$ é definido como o vetor de norma $\ell_{2}$ unitária que maximiza a estimativa para a direção $\mathbf{u}$, assumindo que $\mathbf{x}=\alpha \mathbf{v}(\mathbf{u})$ para um certo $\mathbf{u}$.

$$
\begin{gathered}
\max _{\mathbf{w}} \mathbb{E}\left\{\mathbf{w}^{H} \mathbf{x} \mathbf{x}^{H} \mathbf{w}\right\} ;\|\mathbf{w}\|_{2}=1, \\
\mathbf{w}_{\text {bartlett }}(\mathbf{u})=\frac{1}{\sqrt{N_{M i c}}} \mathbf{v}(\mathbf{u}) .
\end{gathered}
$$

\subsubsection{DAS-KAT}

O algoritmo DAS-KAT implementado realiza a estimação de $\hat{\mathbf{Y}}$ a partir de $\mathbf{X}$ aproveitando o fato de que $\mathbf{W}=\alpha \mathbf{V}$ e que, no arranjo separável, o vetor $\mathbf{V}$ pode ser decomposto em produto de Kronecker da forma $\mathbf{V}=\mathbf{V}_{x} \otimes \mathbf{V}_{y}$ como visto na seção 2.3. Isso nos permite acelerar o cálculo utilizando a Transformada de Kronecker, da forma

$$
\hat{\mathbf{y}} \propto \mathbf{V}^{H} \mathbf{x}=\left(\mathbf{V}_{x} \otimes \mathbf{V}_{y}\right)^{H} \mathbf{x}
$$




$$
\hat{\mathbf{Y}}=\mathbf{V}_{y}^{H} \mathbf{X} \mathbf{V}_{x}^{*}
$$

Pode ser incluído o fator $1 / N_{\text {Mic }}$ para compensação de ganho. Essa formulação reduz a complexidade computacional do problema de $\mathcal{O}\left(N_{\text {Mic }} N_{\text {Dir }}\right)$ para $\mathcal{O}\left(N_{\text {Mic }} \sqrt{N_{\text {Dir }}}+\right.$ $\left.\sqrt{N_{M i c}} N_{D i r}\right)$

\subsubsection{CSM-KAT}

Existem técnicas para estimar uma imagem acústica $\hat{\mathscr{Y}}$ sem passar por uma estimativa do sinal $\hat{\mathbf{Y}}$ [3]. O método que descrevemos aqui estima uma imagem acústica diretamente através da matriz de autocorrelação (Cross Spectral Matrix - CSM) $\mathbf{R}_{\mathbf{x}}$ definida por $\mathbf{R}_{\mathbf{x}}=\mathbb{E}\left\{\mathbf{x x}^{H}\right\}$ [4]. Nesse caso, uma relação entre os produtos de Kronecker e de KhatriRao pode ser explorada para acelerar os cálculos.

Ao utilizar a matriz de autocorrelação como ponto de partida, não há mais a necessidade de armazenar todo o sinal capturado, mas por outro lado, perdemos informações que nos permitiriam estimar o sinal incidente $\hat{\mathbf{Y}}$.

Ignorando a presença de ruído e definindo $\mathbf{R}_{\mathbf{y}}$ como a CSM de $\mathbf{y}$, obtemos a partir de (2.6) que [4]:

$$
\begin{aligned}
\mathbf{R}_{\mathbf{x}} & =\mathbb{E}\left\{\mathbf{x} \mathbf{x}^{H}\right\} \\
& =\mathbb{E}\left\{\mathbf{V} \mathbf{y} \mathbf{y}^{H} \mathbf{V}^{H}\right\} \\
& =\mathbf{V} \mathbb{E}\left\{\mathbf{y} \mathbf{y}^{H}\right\} \mathbf{V}^{H} \\
& =\mathbf{V} \mathbf{R}_{\mathbf{y}} \mathbf{V}^{H} .
\end{aligned}
$$

Normalmente, métodos baseados na CSM assumem que as fontes incidentes são descorrelacionadas. Esse último fator faria $\mathbf{R}_{\mathbf{y}}$ uma matriz diagonal, sendo que os elementos de sua diagonal comporiam $y=\operatorname{vecd}\left\{\mathbf{R}_{\mathbf{y}}\right\}$. Seja $\mathbf{P}$ uma matriz diagonal, $\operatorname{vecd}\{\mathbf{P}\}$ é o vetor constituído pelos elementos da diagonal da matriz quadrada $\mathbf{P}$, e para matrizes $\mathbf{A}_{m \times q}$ e $\mathbf{B}_{n \times q}$ arbitrárias, $\mathbf{A} \odot \mathbf{B}$ é o produto de Khatri-Rao em colunas, definido como

$$
\mathbf{A} \odot \mathbf{B}=\left[\begin{array}{l:l:l:l}
\mathbf{a}_{1} \otimes \mathbf{b}_{1} & \mathbf{a}_{2} \otimes \mathbf{b}_{2} & \cdots & \mathbf{a}_{q} \otimes \mathbf{b}_{q}
\end{array}\right]
$$

com $\mathbf{a}_{i}$ e e $\mathbf{b}_{i}$ representando a i-ésima coluna de $\mathbf{A}$ e $\mathbf{B}$, respectivamente. Podemos então acelerar os cálculos explorando a relação [12]

$$
\operatorname{vec}\left\{\mathbf{B P A}^{T}\right\}=(\mathbf{A} \odot \mathbf{B}) \operatorname{vecd}\{\mathbf{P}\}
$$


Exploraremos também a relação entre o produto de Kronecker e de Khatri-Rao [4]

$$
(\mathbf{A} \otimes \mathbf{B}) \odot(\mathbf{C} \otimes \mathbf{D})=\mathbf{\Xi}[(\mathbf{A} \odot \mathbf{C}) \otimes(\mathbf{B} \odot \mathbf{D})]
$$

onde $\boldsymbol{\Xi}$ é uma matriz de permutação que opera transformando colunas de dimensão $[m n, 1]$ em blocos de dimensão $[m, n]$, como ilustrado na figura 4.

\begin{tabular}{|c|c|c|}
\hline$T_{0,0}$ & $T_{0,1}$ & $T_{0,2}$ \\
\hline & & \\
\hline$T_{1,0}$ & $T_{1,1}$ & $T_{1,2}$ \\
\hline$T_{2,0}$ & $T_{2,1}$ & $T_{2,2}$ \\
\hline
\end{tabular}

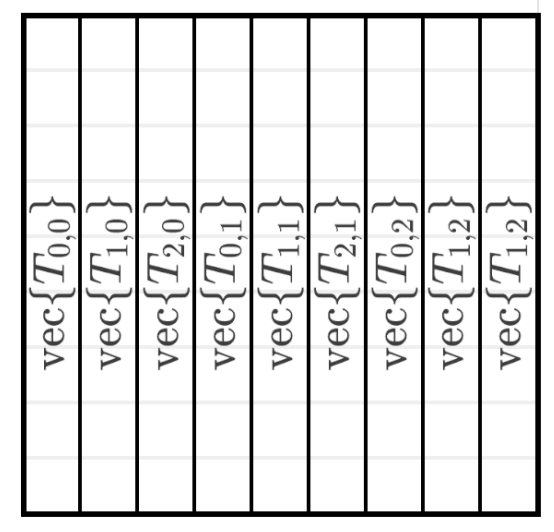

Figura 4: Permutação realizada pela matriz $\boldsymbol{\Xi}$.

Definimos então uma matriz $\mathbf{Z}$ tal que

$$
\operatorname{vec}\left\{\mathbf{R}_{\mathbf{x}}\right\}=\boldsymbol{\Xi} \operatorname{vec}\{\mathbf{Z}\}
$$

sendo $\mathbf{R}_{\mathbf{x}}$ uma matriz quadrada de dimensões $\left[N_{\text {Mic }} \times N_{\text {Mic }}\right]$, cada coluna da matriz $\mathbf{Z}$ será um bloco da matriz $\mathbf{R}_{\mathbf{x}}$ de dimensão $\left[N_{M i c X} \times N_{M i c Y}\right]$ empilhada em um vetor.

Aplicando as relações $(3.13,3.14,3.15)$ a $(3.11)$, pode-se mostrar que

$$
\operatorname{vec}\left\{\mathbf{R}_{\mathbf{x}}\right\}=\boldsymbol{\Xi}\left(\left(\mathbf{V}_{x}^{*} \odot \mathbf{V}_{x}\right) \otimes\left(\mathbf{V}_{y}^{*} \odot \mathbf{V}_{y}\right)\right) \boldsymbol{y}
$$

Definindo então $\tilde{\mathbf{V}}_{x}=\mathbf{V}_{x}^{*} \odot \mathbf{V}_{x}$ e $\tilde{\mathbf{V}}_{y}=\mathbf{V}_{y}^{*} \odot \mathbf{V}_{y}$ :

$$
\operatorname{vec}\left\{\mathbf{R}_{\mathbf{x}}\right\}=\boldsymbol{\Xi} \operatorname{vec}\left\{\tilde{\mathbf{V}}_{y} \mathcal{Y} \tilde{\mathbf{V}}_{x}^{T}\right\}
$$

resulta

$$
\mathbf{Z}=\tilde{\mathbf{V}}_{y} \mathcal{Y} \tilde{\mathbf{V}}_{x}^{T}
$$

Nossa imagem estimada por beamformer passa a ser:

$$
\hat{\mathscr{Y}}=\tilde{\mathbf{V}}_{y}^{H} \mathbf{Z} \tilde{\mathbf{V}}_{x}^{*}
$$




\subsubsection{Beamformer MVDR}

O beamformer Minimum variance distortionless response (MVDR), também conhecido como beamformer Capon [1], modela no domínio da frequência o sinal $\mathbf{x}$ recebido pelos microfones como sinal de interesse $\mathbf{x}_{s}$, assumido determinístico, somado a ruído Gaussiano de média zero n, da forma

$$
\begin{aligned}
\mathbf{x} & =\mathbf{x}_{s}+\mathbf{n} \\
& =\mathbf{V} \mathbf{y}+\mathbf{n} .
\end{aligned}
$$

O objetivo do beamformer MVDR é minimizar a variância do nosso estimador para o sinal de campo distante, que para cada direção $\mathbf{u}$ é $\hat{y}=\mathbf{w}^{H} \mathbf{x}$ (equação (3.1)), sem distorcer o sinal de interesse vindo da direção $\mathbf{u}$. Como $\mathbf{V}$ e $\mathbf{x}_{s}$ são determinísticos, a variância da nossa saída será

$$
\begin{aligned}
\mathbb{E}\left\{|\hat{y}-\mathbb{E}\{\hat{y}\}|^{2}\right\} & =\mathbb{E}\left\{\left|\mathbf{w}^{H} \mathbf{n}\right|^{2}\right\} \\
& =\mathbf{w}^{H} \mathbb{E}\left\{\mathbf{n} \mathbf{n}^{H}\right\} \mathbf{w} \\
& =\mathbf{w}^{H} \mathbf{S}_{n} \mathbf{w} .
\end{aligned}
$$

Sendo $\mathbf{u}_{i}$ o vetor da direção de interesse, impomos ao vetor de pesos $\mathbf{w}$ que $\mathbf{w}^{H}\left(\mathbf{u}_{i}\right) \mathbf{v}\left(\mathbf{u}_{i}\right)=$ 1 para satisfazer o critério de não-distorção. O vetor w será dado por [1]

$$
\begin{gathered}
\mathbf{w}_{M V D R}=\operatorname{argmin}_{\mathbf{w}} \mathbf{w}^{H} \mathbf{S}_{n} \mathbf{w}, \quad \mathbf{w}^{H} \mathbf{v}=1, \\
\mathbf{w}_{M V D R}^{H}=\frac{\mathbf{v}^{H} \mathbf{S}_{n}^{-1}}{\mathbf{v}^{H} \mathbf{S}_{n}^{-1} \mathbf{v}} .
\end{gathered}
$$

Na prática, $\mathbf{S}_{n}$ é desconhecido e utiliza-se uma estimativa a partir de amostras capturadas. Para garantir robustez na inversão matricial, é recomendada uma prática conhecida por diagonal loading [13], utilizando a matriz de covariância somada a um fator diagonal $\mathbf{S}_{n}+\alpha \mathbf{I}$. Mesmo no caso de um sistema separável, a matriz de pesos $\mathbf{W}_{M V D R}$ não é decomponível em produto de Kronecker para acelerarmos as computações via KAT, a não ser no caso excepcional em que $\mathbf{S}_{n}$ for uma matriz diagonal.

Na implementação do MVDR realizada no capítulo 6, foi ignorado completamente Vy para os cálculos de w, mesmo que haja mais de uma fonte de interesse no campo. Utilizamos então a mesma matriz de autocorrelação do ruído para todos os cálculos, aceitando a interferência de uma fonte considerada de interesse na estimativa de outros pixels. Escolheu-se fazer os cálculos desta maneira já que, como o objetivo é estimar uma 
imagem acústica, supõe-se que as direções dos sinais de interesse não são conhecidas $a$ priori, assim os resultados marcados como MVDR nas simulações suprimem bem o ruído de observação, mas não interferências de uma fonte em outra resultantes da filtragem espacial.

\subsubsection{Beamformer MPDR}

O beamformer Minimum power distortionless response (MPDR) é semelhante ao MVDR (seção anterior) mas ao invés de minimizar a variância da saída, visa minimizar sua potência $\mathbb{E}\left\{|\hat{y}|^{2}\right\}$ dentro da mesma restrição de não-distorção. Isso nos leva a

$$
\begin{gathered}
\mathbb{E}\left\{|\hat{y}|^{2}\right\}=\mathbb{E}\left\{\left|\mathbf{w}^{H} \mathbf{x}\right|^{2}\right\} \\
=\mathbf{w}^{H} \mathbb{E}\left\{\mathbf{x} \mathbf{x}^{H}\right\} \mathbf{w} \\
=\mathbf{w}^{H} \mathbf{S}_{x} \mathbf{w}, \\
\mathbf{w}_{M P D R}=\operatorname{argmin}_{\mathbf{w}} \mathbf{w}^{H} \mathbf{S}_{x} \mathbf{w}, \quad \mathbf{w}^{H} \mathbf{v}=1, \\
\mathbf{w}_{M P D R}^{H}=\frac{\mathbf{v}^{H} \mathbf{S}_{x}^{-1}}{\mathbf{v}^{H} \mathbf{S}_{x}^{-1} \mathbf{v}} .
\end{gathered}
$$

Muitas vezes é utilizado o MPDR pela dificuldade de se estimar $\mathbf{S}_{n}$ para o MVDR. Assim como o MVDR, é comum regularizar a matriz $\mathbf{S}_{x}$, somando uma componente em sua diagonal [13]. Em geral, mesmo no caso de um sistema separável, a matriz de pesos $\mathbf{W}_{M P D R}$ também não é decomponível em produto de Kronecker para acelerarmos as computações via KAT.

Repare que, quando temos uma única fonte de interesse e estamos olhando para a direção dessa fonte,

$$
\mathbf{S}_{x}=\sigma_{s}^{2} \mathbf{v} \mathbf{v}^{H}+\mathbf{S}_{n}
$$

e portanto

$$
\begin{aligned}
\mathbf{w}^{H} \mathbf{S}_{x} \mathbf{w} & =\sigma_{s}^{2} \mathbf{w}^{H} \mathbf{v} \mathbf{v}^{H} \mathbf{w}+\mathbf{w}^{H} \mathbf{S}_{n} \mathbf{w} \\
& =\sigma_{s}^{2}+\mathbf{w}^{H} \mathbf{S}_{n} \mathbf{w} .
\end{aligned}
$$

Logo,

$$
\operatorname{argmin}_{\mathbf{w}} \mathbf{w}^{H} \mathbf{S}_{x} \mathbf{w}=\operatorname{argmin}_{\mathbf{w}} \mathbf{w}^{H} \mathbf{S}_{n} \mathbf{w},
$$

e o beamformer MPDR recai no MVDR (seção 3.1.4). 


\subsection{Deconvolução}

A partir das equações (2.6) e (3.3), podemos verificar que a estimativa $\hat{\mathbf{y}}$ obtida por beamforming será igual ao vetor verdadeiro y multiplicado pelo fator $\mathbf{W}^{H} \mathbf{V}$ :

$$
\begin{gathered}
\mathbf{x}=\mathbf{V} \mathbf{y} \\
\hat{\mathbf{y}}=\mathbf{W}^{H} \mathbf{x} \\
\hat{\mathbf{y}}=\mathbf{W}^{H} \mathbf{V} \mathbf{y} .
\end{gathered}
$$

Como o posto da matriz $\mathbf{W}^{H} \mathbf{V}$ não é pleno, não podemos invertê-la para resolver o sistema analiticamente. Devemos então procurar uma maneira de aproximar uma solução.

Considerando como a única fonte no campo sendo um impulso unitário no domínio da frequência vindo de uma direção $\mathbf{u}_{0}, \mathbf{y}$ passa a ser um vetor coluna com apenas um elemento não nulo, sendo esse elemento igual a 1 na linha referente a $\mathbf{u}_{0}$. Nossa estimativa para esse caso é equivalente a

$$
\hat{\mathbf{y}}=\mathbf{W}^{H} \mathbf{v}\left(\mathbf{u}_{0}\right)
$$

o que podemos considerar como uma resposta ao impulso, atrelada à frequência $\omega$ e à direção $\mathbf{u}_{0}$, representada em uma imagem por

$$
\left|\hat{y}\left(\mathbf{u}_{i}\right)\right|^{2}=\mathbf{w}^{H}\left(\mathbf{u}_{i}\right) \mathbf{v}\left(\mathbf{u}_{0}\right) \mathbf{v}^{H}\left(\mathbf{u}_{0}\right) \mathbf{w}\left(\mathbf{u}_{i}\right)
$$

Uma matriz contendo os elementos de $\mathbf{w}^{H}\left(\mathbf{u}_{i}\right) \mathbf{v}\left(\mathbf{u}_{0}\right) \mathbf{v}^{H}\left(\mathbf{u}_{0}\right) \mathbf{w}\left(\mathbf{u}_{i}\right)$ para todas as direções $\mathbf{u}_{i}$ definidas, seguindo a ordenação de $\mathbf{u}_{i}$ em $\mathbf{Y}$ é chamada de Point Spread Function (PSF) e representa a resposta na imagem do beamformer a um impulso incidente da direção $\mathbf{u}_{0}$. Como $\mathbf{v}\left(\mathbf{u}_{i}\right)$ em geral não é ortogonal a $\mathbf{v}\left(\mathbf{u}_{0}\right)$ para $\mathbf{u}_{i} \neq \mathbf{u}_{0}$, o valor da estimativa $\left|\hat{y}\left(\mathbf{u}_{i}\right)\right|^{2}$ é em geral diferente do valor ideal zero, que representaria ausência de interferência. Nessa sessão trataremos sobre algumas implementações do algoritmo de deconvolução DAMAS2, que é estado da arte para compensar o fator $\mathbf{W}^{H} \mathbf{V}$ não ideal na estimativa. DAMAS2 atinge esse objetivo aproximando $\mathbf{W}^{H} \mathbf{V}$ por uma convolução espacial e aplicando uma técnica de deconvolução $[2,11]$.

\subsubsection{Implementação por PSF}

Podemos modelar a imagem estimada pelo beamformer como a imagem ideal afetada pela resposta ao impulso, ou point-spread function (PSF), de cada direção mapeada, da 
forma

$$
\hat{y}=\sum_{i=0}^{N_{D i r}} \mathscr{Y}\left[\mathbf{u}_{i}\right] \operatorname{PSF}\left(\mathbf{u}_{i}\right),
$$

onde $\mathcal{Y}\left[\mathbf{u}_{i}\right]$ representa o valor ideal do pixel atrelado à direção $\mathbf{u}_{i}$. A PSF é respresentada por uma matriz $N_{\text {Dir } X} \times N_{\text {DirY }}, \operatorname{PSF}\left(\mathbf{u}_{0}\right)$ denota a resposta a um impulso vindo da direção $\mathbf{u}_{0}$, e $\mathbf{P S F}\left(\mathbf{u}_{0}\right)\left[\mathbf{u}_{n}\right]$ está acessando na matriz $\mathbf{P S F}\left(\mathbf{u}_{0}\right)$ o elemento referente à direção $\mathbf{u}_{n}$.

No entanto, para um beamformer DAS, e caso o campo seja discretizado com $u_{x}$ e $u_{y}$ uniformemente distribuídos em $[-1,1]$, podemos aproximar a PSF de cada direção como a PSF da direção central $\mathbf{u}_{c}=(0,0,1)$ com sua origem deslocada $[2,10]$, de modo que $\hat{y}$ passa a ser aproximado por uma convolução (veja detalhes em (3.41-3.42) abaixo)

$$
\hat{\mathscr{Y}}=\mathscr{Y}\left[\mathbf{u}_{i}\right] * \operatorname{PSF}\left(\mathbf{u}_{i}\right)
$$

Repare que a aproximação é melhor próximo da direção central $\mathbf{u}_{c}=(0,0,1)$ da imagem.

Descrita aqui é a implementação inicial do algoritmo DAMAS2 proposta em [2]. A PSF para todas as direções é aproximada para a PSF da incidência normal. Cada um de seus elementos é calculado por:

$$
\operatorname{PSF}\left(\mathbf{u}_{c}\right)[\mathbf{u}]=\mathbf{w}^{H}(\mathbf{u}) \mathbf{v}\left(\mathbf{u}_{c}\right) \mathbf{v}^{H}\left(\mathbf{u}_{c}\right) \mathbf{w}(\mathbf{u})=\left|\mathbf{w}^{H}(\mathbf{u}) \mathbf{v}\left(\mathbf{u}_{c}\right)\right|^{2},
$$

onde $\operatorname{PSF}\left(\mathbf{u}_{c}\right)[\mathbf{u}]$ representa o valor do pixel referente à direção $\mathbf{u}$ na imagem quando incide somente um sinal vindo da direção normal (central) $\mathbf{u}_{c} \cdot \mathbf{v}\left(\mathbf{u}_{c}\right)$ é o vetor diretor $\mathbf{v}$ para $\left(u_{x}, u_{y}\right)=(0,0)$.

Para o beamformer DAS em que $\mathbf{w}_{D A S}(\mathbf{u})=\frac{1}{N_{M i c}} \mathbf{v}(\mathbf{u})$ (eq. (3.5)) com a discretização uniforme do espaço, mostramos a seguir que $\operatorname{PSF}\left(\mathbf{u}_{m}\right)\left[\mathbf{u}_{n}\right]=\operatorname{PSF}\left(\mathbf{u}_{m}+\Delta\right)\left[\mathbf{u}_{n}+\Delta\right]$ :

$$
\begin{aligned}
\operatorname{PSF}\left(\mathbf{u}_{m}\right)\left[\mathbf{u}_{n}\right] & =\left|\mathbf{w}^{H}\left(\mathbf{u}_{n}\right) \mathbf{v}\left(\mathbf{u}_{m}\right)\right|^{2} \\
& =\frac{1}{N_{M i c}^{2}}\left|\mathbf{v}^{H}\left(\mathbf{u}_{n}\right) \mathbf{v}\left(\mathbf{u}_{m}\right)\right|^{2} \\
& =\frac{1}{N_{M i c}^{2}}\left|\sum_{l=1}^{N_{M i c}} e^{\frac{j \omega}{c}\left(\mathbf{u}_{n}^{T} \mathbf{p}_{i}-\mathbf{u}_{m}^{T} \mathbf{p}_{i}\right)}\right|^{2} \\
& =\frac{1}{N_{M i c}^{2}}\left|\sum_{i=1}^{N_{M i c}} e^{\frac{j \omega}{c}\left(\left(u_{x_{n}}-u_{x_{m}}\right) p_{x_{i}}+\left(u_{y_{n}}-u_{y_{m}}\right) p_{y_{i}}\right)}\right|^{2} \\
& =\frac{1}{N_{M i c}^{2}} \mid \sum_{i=1}^{N_{M i c}} e^{\left.\frac{j \omega}{c}\left(\left(u_{x_{n}+\Delta_{x}}-u_{x_{m}+\Delta_{x}}\right) p_{x_{i}}+\left(u_{y_{n}+\Delta_{y}}-u_{y_{m}+\Delta_{y}}\right) p_{y_{i}}\right)\right|^{2}} \\
& =\operatorname{PSF}\left(\mathbf{u}_{m}+\boldsymbol{\Delta}\right)\left[\mathbf{u}_{n}+\mathbf{\Delta}\right] .
\end{aligned}
$$


A passagem de (3.42) para (3.43) só é possível porque a distribuição é uniforme e, dadas as direções $x$ discretas do sistema, $x_{m}-x_{n}=x_{m+\Delta}-x_{n+\Delta}$. O mesmo vale para as direções em $y$.

Contudo, à medida em que nos afastamos da direção central nossa aproximação se deteriora, pois passam a faltar pixels da PSF de referência no translado, como ilustrado na figura 5. A aproximação da resposta ao impulso de outras direções para a resposta da direção central reduz a complexidade de memória do programa, pois podemos armazenar somente a resposta correspondente à direção central. Além disso, os $\boldsymbol{\Delta}$ à esquerda e à direita de (3.44) são diferentes na terceira coordenada: enquanto $\Delta_{x}$ e $\Delta_{y}$ forem pequenos o suficiente, é possível ajustar a terceira coordenada de $\Delta$ de maneira que $\mathbf{u}_{m}+\boldsymbol{\Delta}_{m}$ ainda tenha comprimento 1 , e $\mathbf{u}_{n}+\boldsymbol{\Delta}_{n}$ também, mantendo $\Delta_{m, x}=\Delta_{n, x}$ e $\Delta_{m, y}=\Delta_{n, y}$. No entanto, quando os valores de $\Delta_{x}$ e $\Delta_{y}$ forem grandes, ou para $\mathbf{u}_{m}$ ou $\mathbf{u}_{n}$ perto das bordas, pode acontecer que $\mathbf{u}_{m}+\boldsymbol{\Delta}_{m}$ ou $\mathbf{u}_{n}+\boldsymbol{\Delta}_{n}$ caiam fora do círculo unitário, e é isso que faz com que a convolução acabe sendo uma aproximação.

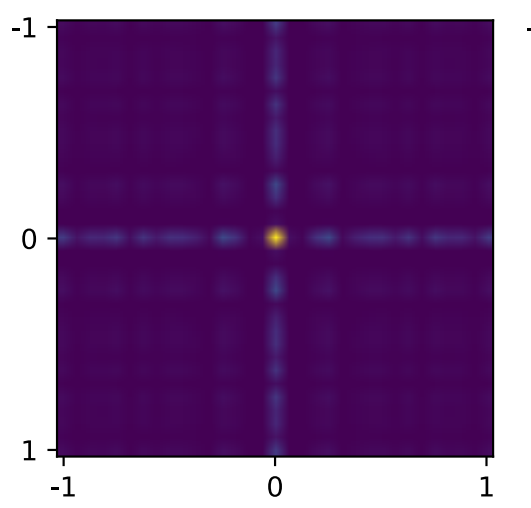

(a)

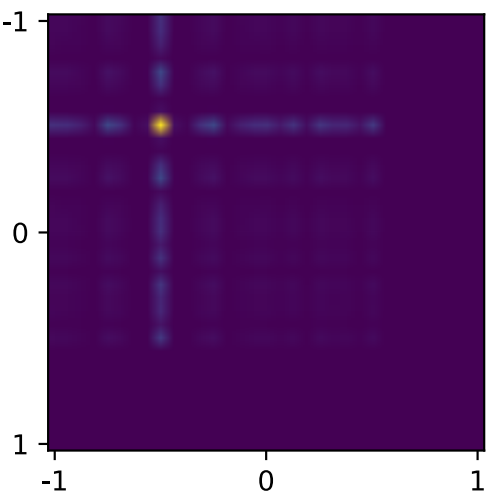

(b)

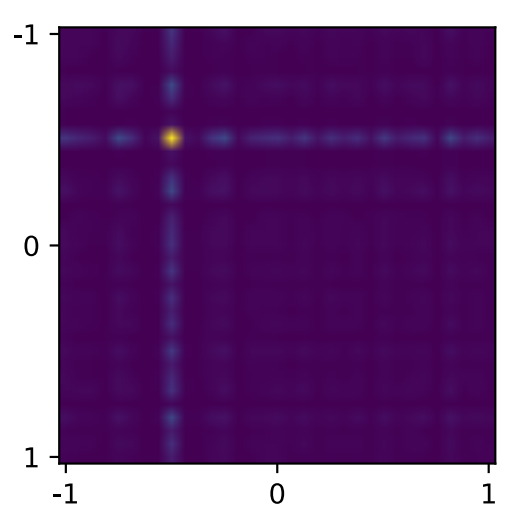

(c)

Figura 5: (a): Point-spread function para a direção central $\operatorname{PSF}\left(\mathbf{u}_{c}\right)$; (b) $\operatorname{PSF}\left(\mathbf{u}_{c}\right)$ deslocada para $\mathbf{u}^{\prime}=\left(\frac{-1}{2}, \frac{-1}{2}, \frac{\sqrt{2}}{2}\right) ; \operatorname{PSF}\left(\mathbf{u}^{\prime}\right)$.

Na prática, a PSF utilizada é aproximada para a resposta do ponto mais próximo do centro disponível no grid da imagem a ser reconstruída, e será utilizado o valor central apenas caso o grid tenha dimensões $[2 n+1 \times 2 m+1], m, n \in \mathbb{N}$ pixels. Se fosse utilizada a aproximação da PSF para a resposta a $\mathbf{u}_{c}=(0,0,1)$ para outros casos em que $N_{\text {Dir }}$ é par, verificou-se que a resposta ao impulso passa a ser menos esparsa e a limpeza dos artefatos da imagem tem precisão reduzida.

Tendo a aproximação para a PSF calculada, é feita a deconvolução da imagem obtida por Beamforming com a PSF calculada pelo algoritmo descrito a seguir. A convolução 
pode ser acelerada por FFT.

\subsubsection{Inicialização}

$$
\begin{aligned}
& \mathcal{Y}^{(0)}=\mathbf{0}_{N_{D i r} \times} \times N_{D i r Y} \\
& a=\sum_{i=0}^{N_{D i r}-1} \operatorname{PSF}\left(\mathbf{u}_{c}\right)
\end{aligned}
$$

\subsubsection{Loop de iteração}

Deve-se realizar essas operações em laço até atingir convergência ou um número máximo de iterações.

$$
\begin{gathered}
\mathbf{B}=\mathscr{Y}^{(k)} * \operatorname{PSF}\left(\mathbf{u}_{c}\right) \\
\mathscr{Y}^{(k+1)}=\max \left(\mathscr{Y}^{(k)}+\frac{1}{a}\left(\mathscr{Y}_{B F}-\mathbf{B}\right), 0\right),
\end{gathered}
$$

onde a operação max é calculada ponto-a-ponto, $\mathcal{Y}^{(k)}$ é a imagem no domínio do espaço ao final da $k$-ésima iteração. $\mathscr{Y}_{B F}$ é a imagem formada pelo beamformer representando as potências de sinal incidentes, * denota convolução, e a constante $a$ é o passo de adaptação, que relaciona velocidade de convergência e qualidade da reconstrução.

O cálculo de $\mathbf{B}$ pode ser acelerado via FFT, desde que se corrija o deslocamento da origem e que se tome medidas para que a convolução não seja cíclica, o que pode ser feito por zero-padding.

\subsubsection{Implementação por matriz A}

Aqui deixaremos de fazer a aproximação $\mathbf{P S F}\left(\mathbf{u}_{i}\right)=\mathbf{P S F}\left(\mathbf{u}_{c}=(0,0,1)\right), \forall i$ ou seja, não consideraremos mais a PSF da direção central como resposta para todas as outras direções. Ao invés disso, definiremos uma matriz $\mathbf{A}$ tal que

$$
\left(\mathbf{A}^{H} \mathbf{A}\right)[:, i]=\operatorname{vec}\left\{\mathbf{P S F}\left(\mathbf{u}_{i}\right)\right\}
$$


em que $(\cdot)[:, i]$ representa a i-ésima coluna de uma matriz, e utilizaremos a real resposta ao impulso de cada direção incidente nos cálculos, portanto esse método é exato. A deconvolução por matriz A não restringe a distribuição das direções $u_{x}$ e $u_{y}$, ao contrário da deconvolução por PSF que depende da distribuição uniforme das direções. Trabalharemos também com variáveis de imagem representadas na forma de matrizes empilhadas em vetores $\operatorname{vec}\{\mathscr{Y}\}$.

Nessa implementação, a operação para cálculo de $\mathbf{b}=\operatorname{vec}\{\mathbf{B}\}$ deixa de ser realizada por convolução como na equação (3.47) e passa a ser realizada como uma operação matricial da forma

$$
\mathbf{b}=\mathbf{A}^{H} \mathbf{A v e c}\{\mathscr{Y}\}=\mathbf{A}^{H} \mathbf{A} y \text {. }
$$

A matriz complexa $\mathrm{A}$, de dimensão $N_{M I C}^{2} \times N_{D i r}$, pode ser derivada utilizando a relação (3.13) em (3.11):

$$
\begin{gathered}
\mathbf{R}_{x}=\mathbf{V} \mathbf{R}_{y} \mathbf{V}^{H} \\
\operatorname{vec}\left\{\mathbf{B} \mathbf{P} \mathbf{A}^{T}\right\}=(\mathbf{A} \odot \mathbf{B})\{\mathbf{P} \\
\operatorname{vec}\left\{\mathbf{R}_{x}\right\}=\operatorname{vec}\left\{\mathbf{V} \mathbf{R}_{y} \mathbf{V}^{H}\right\} \\
=\left(\mathbf{V}^{*} \otimes \mathbf{V}\right) \operatorname{vec}\left\{\mathbf{R}_{y}\right\} \\
=\left(\mathbf{V}^{*} \odot \mathbf{V}\right) \operatorname{vecd}\left\{\mathbf{R}_{y}\right\} \\
=\mathbf{A} \operatorname{vecd}\left\{\mathbf{R}_{y}\right\},
\end{gathered}
$$

em que as duas últimas igualdades valem se $\mathbf{R}_{y}$ for diagonal.

Agora, notando que o estimador de um pixel da imagem acústica usando o beamformer DAS é tal que

$$
\begin{aligned}
\hat{y}\left(\mathbf{u}_{n}\right) & =\frac{1}{N_{M i c}^{2}} \mathbf{v}^{H}\left(\mathbf{u}_{n}\right) \mathbf{R}_{x} \mathbf{v}\left(\mathbf{u}_{n}\right) \\
& =\frac{1}{N_{M i c}^{2}}\left(\mathbf{v}^{T}\left(\mathbf{u}_{n}\right) \otimes \mathbf{v}^{H}\left(\mathbf{u}_{n}\right)\right) \operatorname{vec}\left\{\mathbf{R}_{x}\right\} \\
& =\frac{1}{N^{2}} \mathbf{A}[:, n]^{H} \operatorname{vec}\left\{\mathbf{R}_{x}\right\},
\end{aligned}
$$

verificamos que usando um beamformer DAS para estimar a imagem acústica temos [11]

$$
\begin{aligned}
\hat{y} & =\frac{1}{N_{M i c}^{2}} \mathbf{A}^{H} \operatorname{vec}\left\{\mathbf{R}_{x}\right\} \\
& =\frac{1}{N_{M i c}^{2}} \mathbf{A}^{H} \mathbf{A} \operatorname{vecd}\left\{\mathbf{R}_{y}\right\} .
\end{aligned}
$$


Podemos utilizar a matriz A na deconvolução para os beamformers Bartlett e DAS, uma vez que, para esses beamformers, cada vetor $\mathbf{w}$ é calculado por $\alpha \mathbf{v}$, sendo $\alpha$ um ganho escalar.

A variação do fator escalar $a$, utilizado para ponderar a iteração do algoritmo DAMAS2 e previamente calculado por (3.46), tem um forte efeito na velocidade de convergência do algoritmo. Para considerar um fator equivalente ao algoritmo DAMAS2 implementado por PSF, descrito na seção (3.2.1), devemos desenvolver um novo valor para $a$, para permitir a comparação entre os algoritmos para o mesmo número de iterações.

Ao analisar a equação (3.49), podemos argumentar que no produto $\mathbf{A}^{H} \mathbf{A}$ temos $N_{\text {Dir }}$ PSFs. Para manter a mesma conotação do fator $a$, um valor adequado para o fator $a$ seria a média entre as somas de todas essas PSFs. Obtemos então o cálculo da equação (3.58).

\subsubsection{Inicialização}

$$
\begin{gathered}
\mathbf{A}=\mathbf{V}^{*} \odot \mathbf{V} \\
y^{(0)}=\mathbf{0}_{N_{D i r} \times 1} \\
a=\frac{1}{N_{D i r}} \sum_{i, j=0}^{N_{D i r}-1}\left(\mathbf{A}^{H} \mathbf{A}\right)[i, j]
\end{gathered}
$$

\subsubsection{Loop de iteração}

Deve-se realizar essas operações em laço até atingir convergência ou um número máximo de iterações.

$$
\begin{gathered}
\mathbf{b}=\mathbf{A}^{H} \mathbf{A} y^{(k)} \\
y^{(k+1)}=\max \left(y^{(k)}+\frac{1}{a}\left(y_{B F}-\mathbf{b}\right), 0\right)
\end{gathered}
$$

O produto matricial $\mathbf{A}^{H} \mathbf{A}$ pode ser pré-calculado para acelerar as iterações. 


\subsubsection{Aceleração via KAT}

Ao utilizar o algoritmo DAMAS2 com a matriz A, exposto na seção 3.2.2 para tratar uma imagem obtida a partir de um arranjo separável de microfones, podemos explorar a separabilidade de sua matriz de vetores diretores $\mathbf{V}$ em um produto de Kronecker na forma da equação (2.8) para acelerar via KAT os cálculos de deconvolução, uma vez que a matriz A para os casos de beamformer DAS e Bartlett é calculada a partir da matriz V somente.

Como

$$
\begin{aligned}
\mathbf{A} & =\mathbf{V}^{*} \odot \mathbf{V} \\
& =\left(\mathbf{V}_{x}^{*} \otimes \mathbf{V}_{y}^{*}\right) \odot\left(\mathbf{V}_{x} \otimes \mathbf{V}_{y}\right),
\end{aligned}
$$

a partir da propriedade (3.14) temos

$$
\mathbf{A}=\Xi\left(\mathbf{V}_{x}^{*} \odot \mathbf{V}_{x}\right) \otimes\left(\mathbf{V}_{y}^{*} \odot \mathbf{V}_{y}\right)
$$

Por simplicidade de notação, definimos $\tilde{\mathbf{V}}_{x}=\left(\mathbf{V}_{x}^{*} \odot \mathbf{V}_{x}\right)$ e $\tilde{\mathbf{V}}_{y}=\left(\mathbf{V}_{y}^{*} \odot \mathbf{V}_{y}\right)$,

$$
\begin{gathered}
\mathbf{A}=\Xi\left(\tilde{\mathbf{V}}_{x} \otimes \tilde{\mathbf{V}}_{y}\right) \\
\mathbf{A}^{H} \mathbf{A}=\left(\tilde{\mathbf{V}}_{x} \otimes \tilde{\mathbf{V}}_{y}\right)^{H} \Xi^{H} \Xi\left(\tilde{\mathbf{V}}_{x} \otimes \tilde{\mathbf{V}}_{y}\right) \\
=\left(\tilde{\mathbf{V}}_{x}^{H} \otimes \tilde{\mathbf{V}}_{y}^{H}\right)\left(\tilde{\mathbf{V}}_{x} \otimes \tilde{\mathbf{V}}_{y}\right) \\
=\left(\tilde{\mathbf{V}}_{x}^{H} \tilde{\mathbf{V}}_{x}\right) \otimes\left(\tilde{\mathbf{V}}_{y}^{H} \tilde{\mathbf{V}}_{y}\right),
\end{gathered}
$$

se substituirmos (3.64) em (3.59), a partir da relação (2.13) chegaremos em

$$
\begin{gathered}
\mathbf{b}=\left(\left(\tilde{\mathbf{V}}_{x}^{H} \tilde{\mathbf{V}}_{x}\right) \otimes\left(\tilde{\mathbf{V}}_{y}^{H} \tilde{\mathbf{V}}_{y}\right)\right) y^{(k)} \\
=\operatorname{vec}\left\{\left(\tilde{\mathbf{V}}_{y}^{H} \tilde{\mathbf{V}}_{y}\right) \mathcal{Y}^{(k)}\left(\tilde{\mathbf{V}}_{x}^{H} \tilde{\mathbf{V}}_{x}\right)^{T}\right\} \\
\mathbf{B}=\left(\tilde{\mathbf{V}}_{y}^{H} \tilde{\mathbf{V}}_{y}\right) \mathcal{Y}^{(k)}\left(\tilde{\mathbf{V}}_{x}^{T} \tilde{\mathbf{V}}_{x}^{*}\right) .
\end{gathered}
$$

Considerando pré-computados os produtos $\mathbf{A}^{H} \mathbf{A}$, $\left(\tilde{\mathbf{V}}_{y}^{H} \tilde{\mathbf{V}}_{y}\right)$, e $\left(\tilde{\mathbf{V}}_{x}^{T} \tilde{\mathbf{V}}_{x}^{*}\right)$, podemos verificar que o custo computacional do cálculo de $\mathbf{b}$ realizado em (3.59) tem complexidade $O\left(N_{D i r}^{2}\right)$, enquanto o cálculo realizado em (3.66) tem complexidade $O\left(N_{D i r Y}^{2} N_{D i r X}+\right.$ $\left.N_{D i r Y} N_{D i r X}^{2}\right)$. Se o campo a ser reconstruído for quadrado, ou seja, $N_{D i r Y}=N_{D i r X}$, teremos um ganho computacional da ordem de $\sqrt{N_{D i r}}$. 


\subsubsection{Inicialização}

$$
\begin{gathered}
\mathcal{Y}^{(0)}=\mathbf{0}_{N_{D i r X} \times N_{D i r}} \\
a=\frac{1}{N_{\text {Dir }}} \sum_{i, j=0}^{N_{D i r}-1}\left(\left(\mathbf{V}_{x}^{H} \mathbf{V}_{x}\right) \otimes\left(\mathbf{V}_{y}^{H} \mathbf{V}_{y}\right)\right)[i, j]
\end{gathered}
$$

\subsubsection{Loop de iteração}

Deve-se realizar essas operações em laço até atingir convergência ou um número máximo de iterações.

$$
\begin{gathered}
\mathbf{B}=\left(\mathbf{V}_{y}^{H} \mathbf{V}_{y}\right) \mathscr{Y}^{(k)}\left(\mathbf{V}_{x}^{T} \mathbf{V}_{x}^{*}\right) \\
\mathscr{Y}^{(k+1)}=\max \left(\mathscr{Y}^{(k)}+\frac{1}{a}\left(\mathscr{Y}_{B F}-\mathbf{B}\right), 0\right)
\end{gathered}
$$

Os produtos $\left(\mathbf{V}_{y}^{H} \mathbf{V}_{y}\right)$ e $\left(\mathbf{V}_{x}^{T} \mathbf{V}_{x}^{*}\right)$ podem ser pré-calculados durante a inicialização para acelerar as iterações. 


\section{RECONSTRUÇÃO ESPARSA}

A equação (2.6), repetida a seguir,

$$
\mathbf{x}(\omega)=\mathbf{V}(\omega) \mathbf{y}(\omega)
$$

define a relação entre o sinal x captado pelos sensores e o sinal y de campo distante. Em geral, o número de direções mapeadas é superior ao número de sensores no arranjo, mas podemos a partir dessa relação estimar y definindo uma função custo para as possíveis soluções e tentar minimizá-la para encontrar uma solução única.

Realizando esse processo de reconstrução para todas as frequências, podemos reconstruir o espectro do sinal de campo distante e obter uma estimativa para o sinal proveniente de cada direção mapeada.

Por definição, a solução mais esparsa para um sistema $\mathbf{A x}=\mathbf{b}$ minimiza a pseudonorma $\ell_{0}$ de $\mathbf{x}$ sob a restrição $\mathbf{A x}=\mathbf{b}$, ou seja,

$$
\hat{\mathbf{x}}=\arg \min _{\mathbf{x}}\|\mathbf{x}\|_{0}: \mathbf{A x}=\mathbf{b}
$$

Representada por $\|\mathbf{x}\|_{0}$, a pseudo-norma $\ell_{0}$ de $\mathbf{x}$ é equivalente ao número de elementos não-nulos no vetor $\mathbf{x}$. No entanto, podem ser definidas outras funções custo, como veremos na seção 4.1.1, que ao serem otimizadas também forçam esparsidade em $\mathbf{x}$.

\subsection{Algoritmos para reconstrução esparsa}

Dado um sistema $\mathbf{A x}=\mathbf{b}$, um problema que encontramos é a recuperação do vetor $\mathbf{x}$ esparso a partir de medidas de $\mathbf{b}$. Como existem infinitas soluções para $\mathbf{x}$, utilizamos a informação a priori de que nossa solução é esparsa [14]. 
Tabela 1: Funções custo de minimização para diferentes métodos de regularização

\begin{tabular}{|lr|}
\hline Método & Minimiza \\
\hline \hline Basis-pursuit & $\|\mathbf{x}\|_{1}: \mathbf{A x}=\mathbf{b}$ \\
Basis-pursuit denoise(BPDN) & $\|\mathbf{x}\|_{1}:\|\mathbf{A x}-\mathbf{b}\|_{2} \leq \sigma$ \\
Lasso & $\|\mathbf{x}\|_{1}+\lambda\|\mathbf{A x}-\mathbf{b}\|_{2}$ \\
Total variation $(\mathrm{TV})$ & $\|\nabla \mathbf{x}\|_{2}: \mathbf{A x}=\mathbf{b}$ \\
\hline
\end{tabular}

\subsubsection{Algoritmos de otimização convexa}

Algoritmos baseados em otimização convexa buscam otimizar uma função convexa $f(\cdot)$ sobre o vetor $\mathbf{x}$ dentro de um subconjunto convexo de $\mathbb{R}^{N}$.

Definimos uma função custo $J(\mathbf{x})$ convexa que promove esparsidade na solução, ou seja, $J(\mathbf{x})$ é pequeno para $\mathbf{x}$ esparso. Podemos então resolver

$$
\min _{\mathbf{x}}\{J(\mathbf{x}): \mathbf{b}=\mathbf{A} \mathbf{x}\}
$$

ou então

$$
\left.\min _{\mathbf{x}}\{J(\mathbf{x}): H(\mathbf{b}, \mathbf{A x})<\epsilon)\right\}
$$

quando consideramos que há ruído na medida. Aqui a função $H$ é uma função custo que penaliza a distância entre $\mathbf{b}$ e $\mathbf{A x}$.

Um caso comum para $J$ e $H$ são $J(\mathbf{x})=\|\mathbf{x}\|_{1}$, a norma $\ell_{1}$ de $\mathbf{x}$, e $H(\mathbf{b}, \mathbf{A x})=$ $\|\mathbf{A x}-\mathbf{b}\|_{2}^{2}$, a norma $\ell_{2}$ ao quadrado do erro entre a medida observada $\mathbf{b}$ e a projeção $\mathbf{A} \mathbf{x}$. Alguns casos notáveis estão detalhados na tabela 1.

Dentre os casos notáveis, a regularização TV (ver tabela 1) não possui uma função custo que promove esparsidade. Ela atua minimizando a derivada da imagem, propondo soluções homogêneas e com poucos artefatos. Essa minimização é interessante ao visualizar um campo em uma resolução muito alta, para evitar que a representação de uma fonte se reduza a um único pixel.

\subsubsection{Algoritmos gulosos}

Ao invés de se resolver um problema de otimização convexa, uma alternativa à recuperação esparsa seria a aproximação esparsa. Ao invés de buscar a solução mais esparsa que satisfaz um problema, abordamos o problema selecionando colunas da matriz de aquisição A e criando aproximações sucessivas melhores para $\mathbf{X}$. 


\subsubsection{OMP}

O algoritmo Orthogonal Matching Pursuit (OMP) busca em cada iteração adicionar ao suporte da solução o elemento correspondente à coluna da matriz de aquisição $\mathbf{A}$ com maior correlação com o vetor de erro residual r. O suporte de um vetor $\mathbf{x}$ são os índices dos elementos não-nulos de $\mathbf{x}$. O resíduo $\mathbf{r}$ é a diferença entre o vetor medido $\mathbf{b}$ e o obtido através da aproximação $\mathbf{A} \hat{\mathbf{x}}$, para uma dada estimativa $\hat{\mathbf{x}}$ para o vetor $\mathbf{x}$. Para um problema do tipo $\mathbf{A x}=\mathbf{b}$, temos que $\mathbf{r}=\mathbf{b}-\mathbf{A} \hat{\mathbf{x}}$.

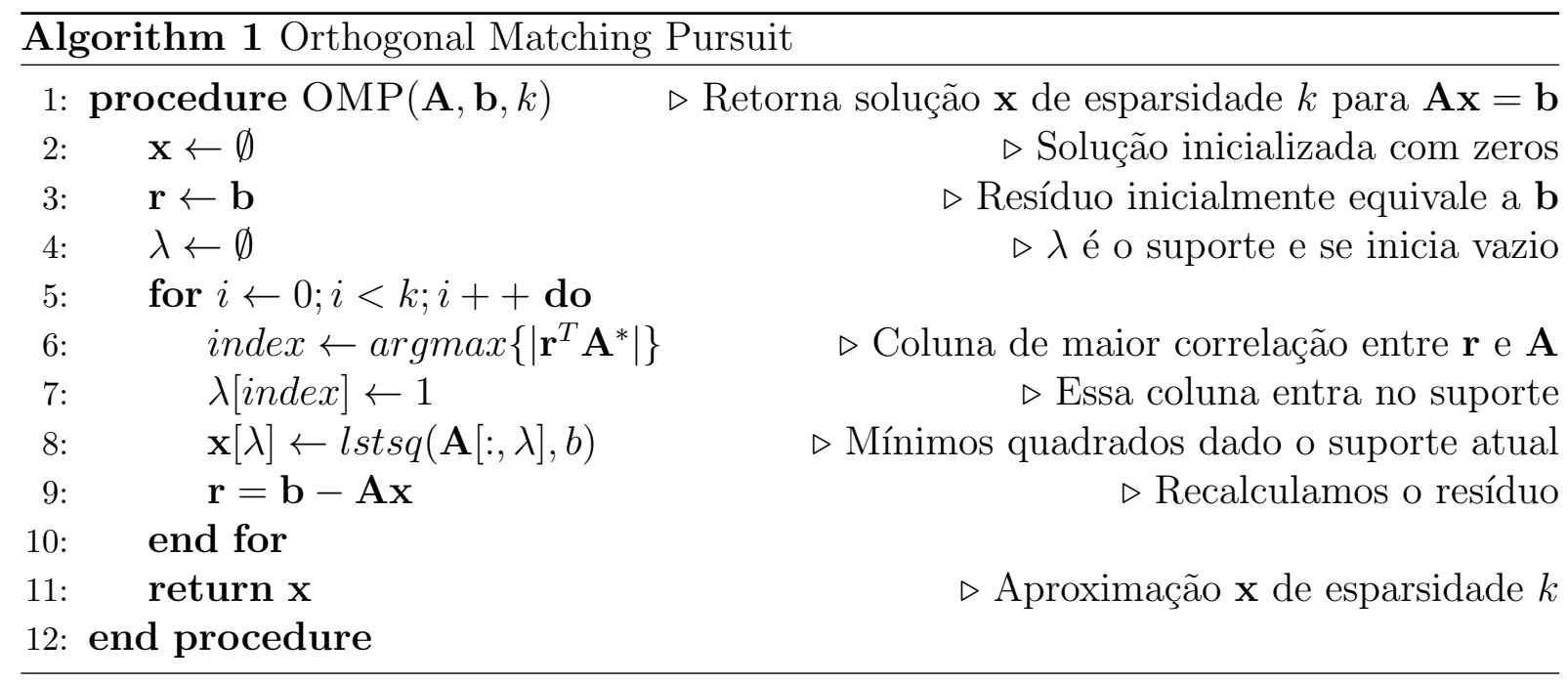

Aqui ilustramos uma implementação direta do algoritmo OMP. O OMP tem a vantagem de que, uma vez dado o número $k$ de elementos não nulos de $\mathbf{x}$, ele retornará a solução em $k$ iterações. Comparações entre implementações otimizadas do OMP podem ser encontradas em [15].

Quando a matriz de aquisição A pode ser decomposta no produto de Kronecker $\mathbf{A}=\mathbf{A}_{x} \otimes \mathbf{A}_{y}(2.7,2.8)$, podemos acelerar o passo 6 onde ocorre o cálculo do índice de suporte a ser adicionado, passando da forma

$$
\text { index } \leftarrow \arg \max \left\{\left|\mathbf{r}^{T} \mathbf{A}^{*}\right|\right\}
$$

para

$$
\text { index } \leftarrow \arg \max \left\{\left|\mathbf{A}_{x}^{H} \mathbf{R} \mathbf{A}_{y}^{*}\right|\right\}
$$

onde $\mathbf{R}$ é o vetor coluna $\mathbf{r}$, originalmente de dimensão $\left[N_{D i r}, 1\right]$, redimensionado para uma matriz $\left[N_{\operatorname{Dir} X}, N_{\operatorname{Dir} Y}\right]$. No caso em que aplicamos o algoritmo ao processamento de um arranjo de microfones, e temos um sistema separável (seção 2.3), podemos explorar essa propriedade para acelerar o OMP. 


\subsection{Parâmetros de reconstrução}

As características do sistema a ser reconstruído estão, pelo nosso modelo, representadas pela matriz de aquisição A. A partir de alguns parâmetros da matriz de aquisição A podemos estimar a qualidade da reconstrução nesse sistema mesmo sem olhar para medições $\mathbf{b}$ ou para o vetor $\mathbf{x}$ que queremos reconstruir, o que exigiria inúmeros ensaios. Como são parâmetros quantitativos que nos trazem garantias quanto à esparsidade ou unicidade da solução, podemos adotá-los como indicadores. Veremos a seguir dois desses parâmetros, o spark e a coerência mútua.

\subsubsection{Spark}

O spark de uma matriz A é o menor número de colunas de $\mathbf{A}$ que são linearmente dependentes entre si. Seu operador é denotado por $\operatorname{spark}(\mathbf{A})$.

$$
\operatorname{spark}(\mathbf{A})=\min \|\mathbf{x}\|_{0} \text { tal que } \mathbf{A} \mathbf{x}=\mathbf{0}, \mathbf{x} \neq \mathbf{0},
$$

onde $\|\mathbf{x}\|_{0}$ representa a pseudonorma $l_{0}$ do vetor $\mathbf{x}$.

Para um sistema da forma $\mathbf{A x}=\mathbf{b}$, o spark nos traz informações sobre a esparsidade de sua solução [16].

Teorema 1. Se $\mathbf{A x}=\mathbf{b}$ possui uma solução $\mathbf{x}$ tal que

$$
\|\mathbf{x}\|_{0}<\frac{\operatorname{spark}(\mathbf{A})}{2}
$$

então $\mathbf{x}$ é a solução mais esparsa para o sistema.

O valor de $\operatorname{spark}(\mathbf{A})$ em geral tem um alto custo para se obter, pois exige uma análise combinatória e tem complexidade computacional não-polinomial.

Para análise de uma matriz proveniente do produto de Kronecker entre duas matrizes que não têm posto pleno com colunas normalizadas, o seu spark pode ser calculado como o valor mínimo entre os sparks das matrizes iniciais [17].

$$
\begin{gathered}
\mathbf{A}=\mathbf{B} \otimes \mathbf{C} \Rightarrow \\
\operatorname{spark}(\mathbf{A})=\min (\operatorname{spark}(\mathbf{B}), \operatorname{spark}(\mathbf{C}))
\end{gathered}
$$




\subsubsection{Coerência}

A coerência mútua de uma matriz $\mathbf{A}$, também chamada simplesmente de coerência, é o maior valor para o produto interno entre duas colunas de A normalizadas. Seu operador é denotado por $\mu(\mathbf{A})$.

$$
\mu(\mathbf{A})=\max \frac{\mathbf{a}_{k}^{T} \mathbf{a}_{j}}{\left\|\mathbf{a}_{k}\right\|_{2}\left\|\mathbf{a}_{j}\right\|_{2}}, k \neq j
$$

Para matrizes unitárias temos $\mu(\mathbf{A})=0$, e para matrizes com mais colunas do que linhas temos necessariamente que $\mu(\mathbf{A})>0$. Para problemas de reconstrução, é interessante que $\mu(\mathbf{A})$ seja pequeno, aproximando-se de uma matrix unitária.

Utilizando a relação

$$
\operatorname{spark}(\mathbf{A}) \geq 1+\mu^{-1}(\mathbf{A})
$$

retirada de [16] e substituindo (4.10) em (4.7) chegamos na relação

$$
\|\mathbf{x}\|_{0}<\frac{1}{2}\left(1+\mu^{-1}(\mathbf{A})\right)
$$

que, se satisfeita, garante que $\mathbf{x}$ é a solução mais esparsa para o problema.

Usamos também de [16] e [18] o seguinte teorema:

Teorema 2. Se A possui colunas normalizadas e $\mathbf{A x}=\mathbf{b}$ possui uma solução $\mathbf{x}$ que satisfaz

$$
\|\mathbf{x}\|_{0}<\frac{1}{2}\left(1+\mu^{-1}(\mathbf{A})\right)
$$

então $\mathbf{x}$ é solução única que minimiza as normas $\ell_{0}$ e $\ell_{1}$.

Para uma matriz proveniente do produto de Kronecker entre matrizes de colunas normalizadas, sua coerência pode ser calculada como o valor máximo entre as coerências das matrizes iniciais [17]:

$$
\begin{gathered}
\mathbf{A}=\mathbf{B} \otimes \mathbf{C} \Rightarrow \\
\mu(\mathbf{A})=\max (\mu(\mathbf{B}), \mu(\mathbf{C})) .
\end{gathered}
$$

Seja $\mathbf{A}=\left[\begin{array}{l:l:l}\mathbf{a}_{1} & \cdots & \mathbf{a}_{n}\end{array}\right]$, sendo que $\mu(\mathbf{A})=\max _{i \neq j} \mathbf{a}_{i}^{T} \mathbf{a}_{j}$. Uma vez que $\mathbf{a}_{i}=\mathbf{b}_{p} \otimes \mathbf{c}_{q}$ $\mathrm{e} \mathbf{a}_{j}=\mathbf{b}_{r} \otimes \mathbf{c}_{s}$, temos

$$
\mathbf{a}_{i}^{T} \mathbf{a}_{j}=\left(\mathbf{b}_{p} \otimes \mathbf{c}_{q}\right)^{T}\left(\mathbf{b}_{r} \otimes \mathbf{c}_{s}\right)=\left(\mathbf{b}_{p}^{T} \mathbf{b}_{r}\right)\left(\mathbf{c}_{q}^{T} \mathbf{c}_{s}\right)
$$


Temos que

$$
\begin{aligned}
\mu(\mathbf{A}) & =\max _{(p, q) \neq(r, s)}\left(\mathbf{b}_{p}^{T} \mathbf{b}_{r}\right)\left(\mathbf{c}_{q}^{T} \mathbf{c}_{s}\right) \\
& =\max \left\{\max _{p \neq r}\left(\mathbf{b}_{p}^{T} \mathbf{b}_{r}\right)\left(\mathbf{c}_{q}^{T} \mathbf{c}_{s}\right), \max _{q \neq s}\left(\mathbf{b}_{p}^{T} \mathbf{b}_{r}\right)\left(\mathbf{c}_{q}^{T} \mathbf{c}_{s}\right),\right\},
\end{aligned}
$$

mas como $\mathbf{B}$ e $\mathbf{C}$ possuem colunas normalizadas,

$$
\left(\mathbf{b}_{p}^{T} \mathbf{b}_{r}\right)\left(\mathbf{c}_{q}^{T} \mathbf{c}_{s}\right) \leq \mathbf{b}_{p}^{T} \mathbf{b}_{r}
$$

e

$$
\left(\mathbf{b}_{p}^{T} \mathbf{b}_{r}\right)\left(\mathbf{c}_{q}^{T} \mathbf{c}_{s}\right) \leq \mathbf{c}_{q}^{T} \mathbf{c}_{s}
$$

Então

$$
\begin{aligned}
\mu(\mathbf{B} \otimes \mathbf{C}) & =\max _{p \neq r, q \neq s}\left\{\mathbf{b}_{p}^{T} \mathbf{b}_{r}, \mathbf{c}_{q}^{T} \mathbf{c}_{s}\right\} \\
& =\max \left\{\max _{p \neq r} \mathbf{b}_{p}^{T} \mathbf{b}_{r}, \max _{q \neq s} \mathbf{c}_{q}^{T} \mathbf{c}_{s}\right\} \\
& =\max \{\mu(\mathbf{B}), \mu(\mathbf{C})\} .
\end{aligned}
$$

Utilizaremos a relação (4.19) na seção 6.2 para acelerar os cálculos da coerência para cada geometria de arranjo, trabalhando com sistemas onde $\mathbf{V}_{x}=\mathbf{V}_{y}$ e extrapolando os resultados de sistemas unidimensionais. 


\section{DISCRETIZAÇÃO DO ESPAÇO}

Tomaremos daqui em diante a medida da coerência da matriz diretora $\mathbf{V}$ como um qualificador do sistema de arranjo. A matriz $\mathbf{V}$ é calculada como uma função do posicionamento dos microfones no arranjo e do conjunto de direções $\mathbf{u}$ no qual discretizamos o espaço. Analizaremos a coerência em função da discretização do espaço e então mostramos como otimizar a discretização para uma região de baixas frequências no arranjo separável.

Neste capítulo provamos que ao discretizar nosso sistema separável de maneira uniforme no espaço, ou seja, ao selecionar os vetores $\mathbf{u}$ tais que $u_{x}$ e $u_{y}$ sejam equi-espaçados entre $[-1,1]$, nós obtemos a coerência mínima da matriz diretora $\mathbf{V}$ para baixas frequências e arranjos separáveis. Primeiro provamos que a discretização uniforme é ótima para sistemas unidimensionais, onde $\mathbf{p}=\left[\begin{array}{lll}p & 0 & 0\end{array}\right]^{T}$ e $\mathbf{u}=\left[\begin{array}{lll}u & \sqrt{1-u^{2}}\end{array}\right]^{T}$, e então generalizamos o resultado para sistemas bidimensionais separáveis, explorando a relação entre coerência e produto de Kronecker da equação (4.13).

Considerando o caso unidimensional, definimos a função $g_{m, n}(\omega)$ como

$$
g_{m, n}(\omega)=\frac{1}{N_{\text {Mic }}^{2}}\left|\mathbf{v}\left(\omega: \mathbf{u}_{m}\right)^{H} \mathbf{v}\left(\omega: \mathbf{u}_{n}\right)\right|^{2}, \quad m \neq n .
$$

Se mantivéssemos a mesma definição para $m=n$, sempre teríamos o valor máximo de $g_{m, n}$ na diagonal e igual a 1 . Por isso definimos que $g_{m, n}=0$ se $m=n$. Dessa maneira, a coerência é $\mu(\mathbf{V})=\max _{m, n} \sqrt{g_{m, n}(\omega)}$. Substituindo $\left[\mathbf{v}\left(\omega: \mathbf{u}_{i}\right)\right]_{k}=e^{-j \frac{\omega}{c} u_{i} p_{k}}$ na equação (5.1), obtemos que

$$
g_{m, n}(\omega)=\frac{1}{N_{\text {Mic }}^{2}} \sum_{k=1}^{N_{\mathrm{Mic}}} \sum_{\ell=1}^{N_{\mathrm{Mic}}} e^{-j \frac{\omega}{c}\left(u_{m}-u_{n}\right)\left(p_{k}-p_{\ell}\right)} .
$$

Note que $g_{m, n}(0)=1$ para todo $m, n$, logo o pior caso para a coerência ocorrerá para baixas frequências. Para cada termo $e^{-j \frac{\omega}{c}\left(u_{m}-u_{n}\right)\left(p_{k}-p_{\ell}\right)}$ do somatório com $k \neq \ell$, existe um único termo $e^{-j \frac{\omega}{c}\left(u_{m}-u_{n}\right)\left(p_{\ell}-p_{k}\right)}$, que é conjugado do primeiro. Uma vez que a soma desses dois termos é $2 \cos \left(\frac{\omega}{c}\left(u_{m}-u_{n}\right)\left(p_{k}-p_{\ell}\right)\right)$ e a soma dos termos com $k=\ell$ é $\frac{1}{N_{\text {Mic }}}$, a 
equação (5.2) pode ser reescrita da forma

$$
g_{m, n}(\omega)=\frac{1}{N_{\text {Mic }}}+\frac{2}{N_{\text {Mic }}^{2}} \sum_{k=1}^{N_{\text {Mic }}} \sum_{\ell=k+1}^{N_{\text {Mic }}} \cos \left(\frac{\omega}{c}\left(u_{m}-u_{n}\right)\left(p_{k}-p_{\ell}\right)\right) .
$$

Considere que $\omega$ é pequeno o suficiente tal que

$$
\mu(\mathbf{V})^{2}=g_{m^{*}, n^{*}}(\omega), \quad\left(m^{*}, n^{*}\right)=\arg \min _{m, n}\left|u_{m}-u_{n}\right| .
$$

A equação (5.4) é verdadeira se $\omega$ é pequeno a ponto que cada cosseno somado em (5.3) seja uma função decrescente em respeito a $\left|u_{m}-u_{n}\right|$, nesse caso $g_{m, n}(\omega)$ seria maximizado ao escolhermos $m$ e $n$ tal que $\left|u_{m}-u_{n}\right|$ seja mínimo. Já que $\mu(\mathbf{V})$ é uma função contínua de $\omega$, (5.4) se mantém verdadeira até que $\omega$ passe a ser grande o suficiente tal que $g_{m, n}(\omega)=$ $g_{m^{*}, n^{*}}(\omega)$ para algum $m \neq m^{*}$ e $n \neq n^{*}$. Dessa forma, $\mu(\mathbf{V})^{2}=g_{m^{*}, n^{*}}(\omega)$ se $0<\omega \leq \omega^{*}$, onde

$$
\omega^{*}=\min _{\omega>0}\left\{\omega: \mu(\mathbf{V})^{2}=g_{m^{*}, n^{*}}(\omega)\right\}
$$

Temos como objetivo obter o melhor conjunto de direções $\left(u_{0}, u_{1}, \cdots, u_{N_{\text {Dir }}-1}\right)$ tal que a coerência $\mu(\mathbf{V})$ seja mínima, o que é atingível ao minimizar $\mu(\mathbf{V})^{2}$. Definindo $\omega_{1}>0$ como o primeiro ponto crítico de $g_{m^{*}, n^{*}}(\omega), \mu(\mathbf{V})^{2}$ é uma função decrescente com respeito a $\Delta u^{*}$ para $\omega<\min \left\{\omega^{*}, \omega_{1}\right\}$, logo, nesta faixa de frequências, $\Delta u^{*}$ deve ser maximizado. Em outras palavras, para $\omega<\min \left\{\omega^{*}, \omega_{1}\right\}$, a coerência será mínima se as direções forem escolhidas como

$$
\left(u_{1}^{*}, \cdots, u_{N_{\text {Dir }}}^{*}\right)=\arg \max _{u_{1}, \cdots, u_{N_{\text {Dir }}}} \min _{i}\left\{u_{i}-u_{i-1}\right\}, \quad-1 \leq u_{1} \leq \cdots \leq u_{N_{\text {Dir }}} \leq 1 .
$$

Chegamos na solução desse problema da seguinte maneira: a equação (5.6) deve satisfazer $u_{i}^{*}-u_{i-1}^{*}=$ constante $\forall i$, dado que, se não fosse constante, $u_{k}^{*}-u_{k-1}^{*}=\min _{i} u_{i}^{*}-u_{i-1}^{*}$ poderia ser elevado por um pequeno aumento no valor de $u_{k}^{*}-u_{k-1}^{*}$ tal que $k$ permaneceria como o índice que minimiza $u_{i}^{*}-u_{i-1}^{*}, \operatorname{logo} u_{k}^{*}-u_{k-1}^{*}$ não maximizaria a expressão. Além disso, para maximizar o menor intervalo entre $u_{i}$ e $u_{i-1}$, a distância entre o maior e o menor elemento de $\left\{u_{i}\right\}_{i=0}^{N_{\text {Dir }}-1}$ deve ser a maior possível. Como todo $u_{i}$ se encontra no intervalo $[-1,1]$, devemos ter $u_{0}^{*}=-1$ e $u_{N_{\text {Dir }}-1}^{*}=1$. Consequentemente, a solução para (5.6) é linearmente espaçada entre -1 e +1 :

$$
u_{i}^{*}=-1+\frac{2}{N_{\text {Dir }}-1} k, \quad 1 \leq k \leq N_{\text {Dir }}
$$

Logo, a discretização do espaço de mínima coerência é uma distribuição uniforme se 
$0<\omega<\min \left\{\omega^{*}, \omega_{1}\right\}$, onde $\omega^{*}$ é dado pela equação (5.5) e $\omega_{1}$ é a menor frequência tal que $\frac{\mathrm{d}}{\mathrm{d} \omega} g_{m^{*}, n^{*}}(\omega)=0$. Apesar de $\omega^{*}$ não possuir uma expressão fechada, ele pode ser calculado numericamente utilizando a equação (5.5). Por exemplo, para a geometria de arranjo não redundante que utilizamos, em que o espaçamento entre quaisquer dois microfones é único (em detalhes na seção 6.2.1), com $N_{\text {Mic }}=8, N_{\text {Dir }}=33$ e $c=343 \mathrm{~m} / \mathrm{s}$, temos $\omega_{1}=2 \pi \times 12.64 \mathrm{krad} / \mathrm{s}$ e $\omega^{*}=2 \pi \times 9.17 \mathrm{krad} / \mathrm{s}$, valores em concordância com os resultados numéricos da seção 6.2 .

Para arranjos separáveis bidimensionais, podemos utilizar a relação entre a coerência e o produto de Kronecker da equação (4.13) $\mu(\mathbf{V})=\max \left\{\mu\left(\mathbf{V}_{x}\right), \mu\left(\mathbf{V}_{y}\right)\right\}$. Cada direção passa a ser dada por $\mathbf{u}=\left[u_{x} u_{y} \sqrt{1-u_{x}^{2}-u_{y}^{2}}\right]^{T}$. O valor mínimo para $\mu(\mathbf{V})$ somente pode ser atingido quando ambos $\mu\left(\mathbf{V}_{x}\right)$ e $\mu\left(\mathbf{V}_{y}\right)$ forem minimizados em relação à discretização do espaço, o que ocorre quando $u_{x}$ e $u_{y}$ seguem a distribuição uniforme entre $[-1,1]$ da equação (5.7). 


\section{RESULTADOS NUMÉRICOS}

Dado o estudo técnico realizado, temos interesse em testar diferentes algoritmos de imageamento e reconstrução de sinal, e diferentes discretizações do espaço para validar os tópicos até agora abordados. Também queremos comparar diferentes geometrias de arranjo, e a construção de diversos arranjos é inviável. Para testar o desempenho dos algoritmos e comparar diferentes sistemas, foi desenvolvido um software que simula o sinal capturado por cada microfone do arranjo e implementa os métodos de reconstrução.

\subsection{Simulador de Imageamento Acústico}

O simulador de Imageamento Acústico, escrito em python 2.7, foi desenvolvido para realizar testes de reconstrução de imagens acústicas a partir de um campo distante discreto, e foi estruturado para poder utilizar sinais no tempo na construção dos campos. Para garantir precisão numérica, todas as variáveis e operações matriciais são implementadas pela biblioteca numpy, com outras funções importadas do módulo scipy.

Utilizando um arranjo separável virtual, pode-se explorar inúmeras geometrias, permitindo análises em função do número de sensores de diferentes posicionamentos. A implementação do imageamento também permite a utilização de diferentes algoritmos de reconstrução para comparar resultados e performance computacional.

Como as funções implementadas de imageamento e reconstrução direcional operam no domínio da frequência, a simulação das medidas tomadas pelo arranjo é feita através de interpolações no tempo, com o intuito de reduzir o viés do modelo adotado pelos algoritmos na simulação. Especialmente para frequências mais altas, a interpolação no tempo pode trazer distorções em relação ao caso real, portanto é recomendado trabalhar com frequências de amostragem significativamente superiores a Nyquist. 
Listagem 6.1: Exemplo de uso da classe FarFieldSignal

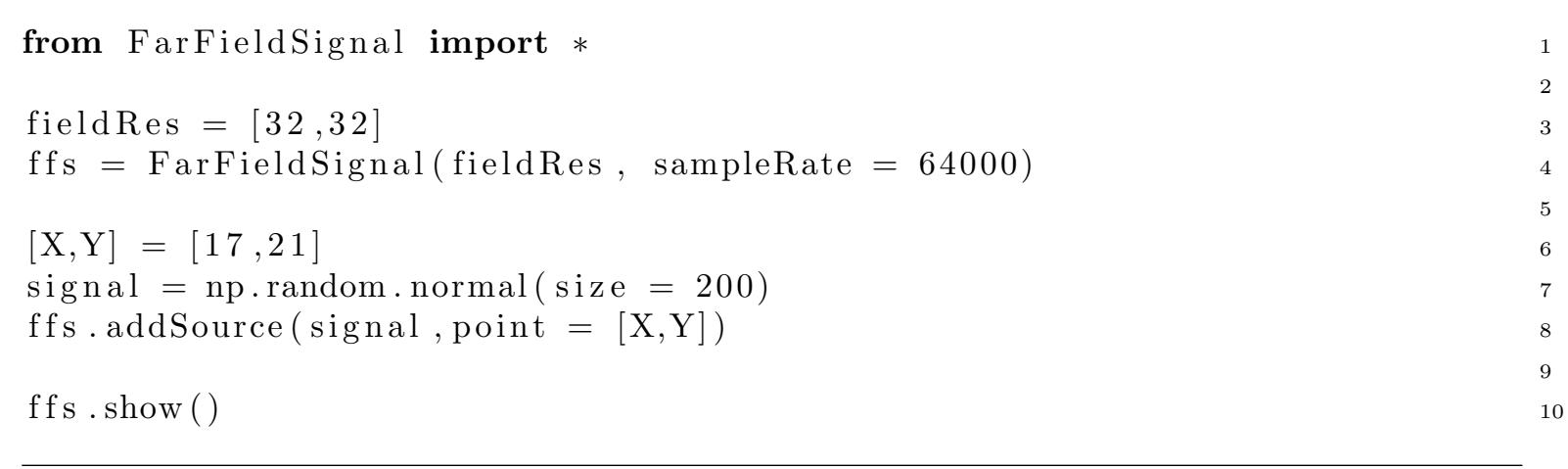

\subsubsection{Estrutura do simulador}

O software do simulador foi estruturado em classes e orientado a objetos, criando uma camada de abstração na sua utilização. Foram estabelecidas 3 classes base, uma para representar o sinal de campo distante, chamada de FarFieldSignal, uma para representar o arranjo separável de sensores contendo os sinais capturados, chamada de GridArray, e outra responsável por executar as rotinas de imageamento e reconstrução a partir das medidas do arranjo, chamada de Imager. Além das classes base, foi também implementada uma classe especializada chamada SignalEstimator, com heranças da classe Imager. Cada classe será detalhada a seguir.

\subsubsection{FarFieldSignal}

A classe FarFieldSignal modela discretamente o campo incidente no arranjo. O trecho de código na listagem 6.1 importa o módulo que contém a classe FarFieldSignal, instancia o objeto ffs como campo distante de resolução discreta $32 \times 32$ e taxa de amostragem $64 \mathrm{kHz}$. Em seguida adiciona uma fonte de ruído branco ao campo distante, na direção representada pelo pixel $(17,21)$. É chamada a função de visualização do campo distante com ffs.show(), que retorna a imagem da figura 6 .

Além disso, ao incluir um sinal no campo distante, pode-se determinar o seu espalhamento através de uma matriz de pesos, ao inves de definí-lo como proveniente de uma fonte pontual. Pode-se também adicionar uma fonte com um sinal proveniente de um arquivo . wav que será reamostrado para a taxa de amostragem estabelecida na instanciação da classe através de uma interpolação cúbica no tempo. 


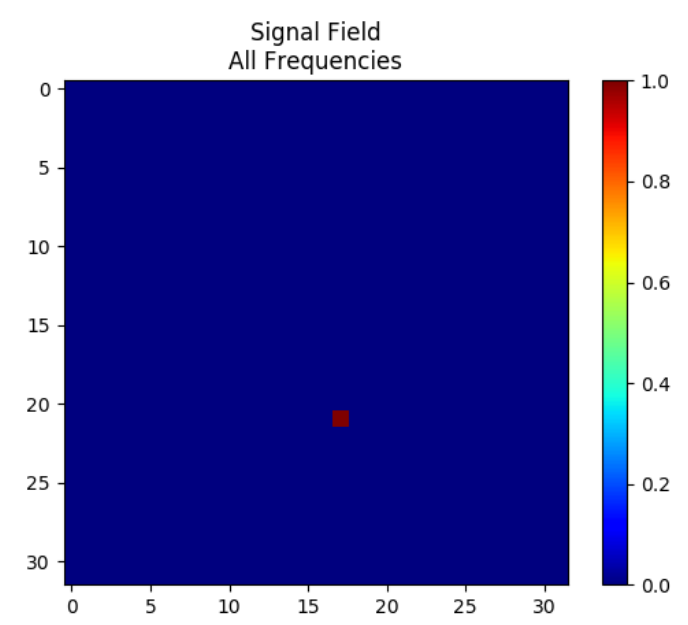

Figura 6: Retorno de ffs.show() para o exemplo de FarFieldSignal.

Listagem 6.2: Exemplo de uso da classe GridArray

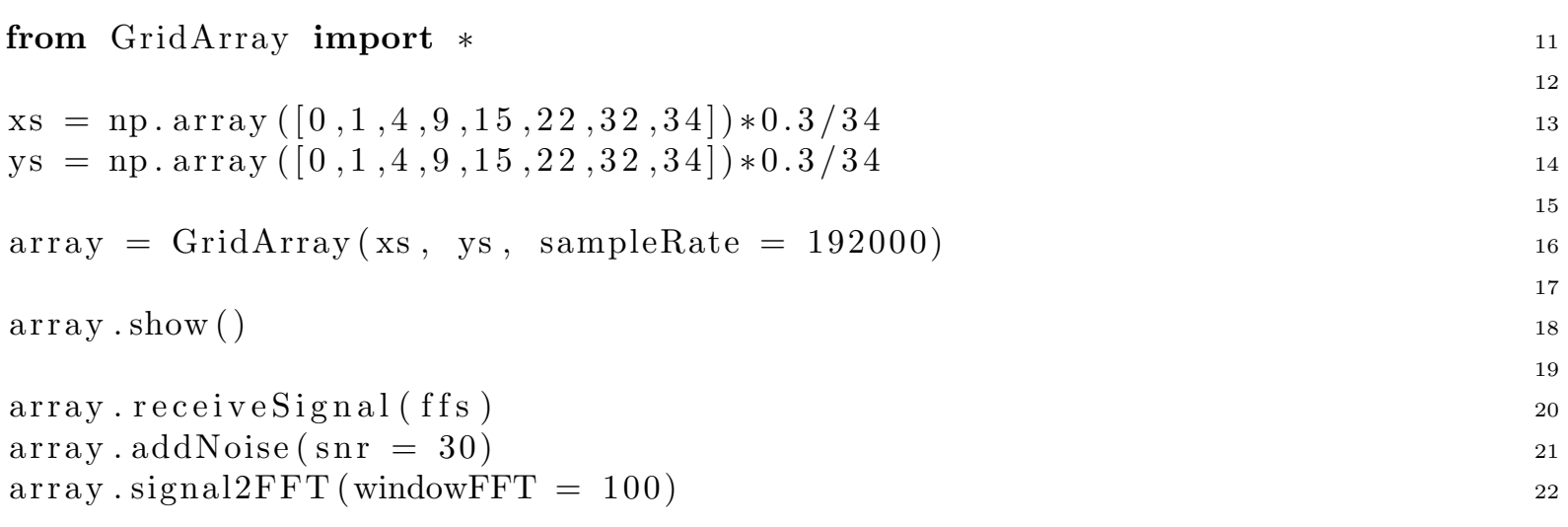

\subsubsection{GridArray}

A classe GridArray modela um arranjo de microfones de configuração separável, onde cada um dos microfones se encontra em um grid XY não necessariamente equi-espaçado.

A listagem 6.2 exemplifica um código que, primeiramente, importa o módulo que contém a classe GridArray, instancia o objeto array como arranjo de configuração não redundante $8 \times 8$ de dimensões $30 \mathrm{~cm} \times 30 \mathrm{~cm}$ e taxa de amostragem $192 \mathrm{kHz}$, e chama a função de visualização do arranjo em array.show(), que retorna a imagem da figura 7 . No caso exemplificado, como houve a reamostragem dos sinais de $64 \mathrm{kHz}$ para $192 \mathrm{kHz}$, teremos 600 amostras no tempo para cada microfone. Definindo o tamanho da janela para realizar a FFT em 100 amostras, teremos um espaçamento de $1920 \mathrm{~Hz}$ entre as amostras na frequência [19].

O arranjo então recebe o campo distante ffs, declarado na listagem (6.1), adiciona 


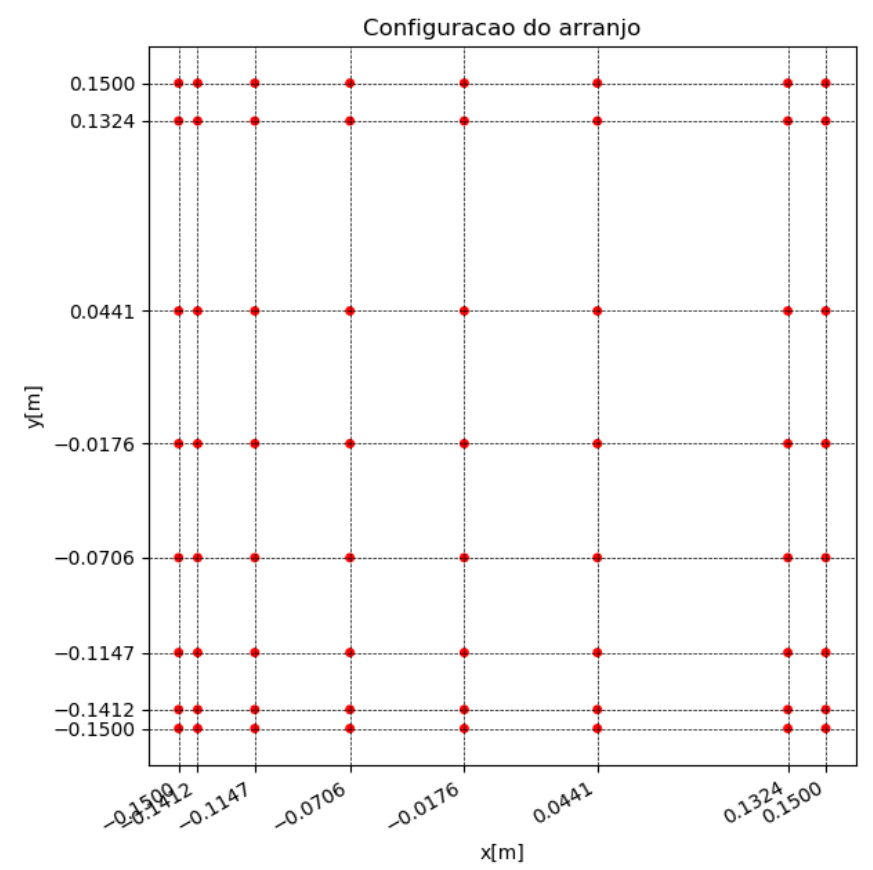

Figura 7: Retorno de array. show() para o exemplo de GridArray.

ruído branco em cada um dos microfones com SNR de $30 \mathrm{~dB}$, referente à potência média que incide em todos os microfones.

O método GridArray.signal2FFT faz o janelamento e a FFT do sinal recebido pelos microfones. Esse método cria o parâmetro Imager .signalFFT contendo 6 janelas do sinal convertido para o domínio da frequência. Como estamos realizando a FFT de um sinal real, seu espectro será simétrico na frequência, então teremos em cada janela 50 valores complexos referentes ao espectro do sinal de 0 a $96 \mathrm{kHz}$.

\subsubsection{Imager}

A classe Imager não modela um objeto real mas funciona como um encapsulamento para as funções de imageamento. Com ela, ao receber um arranjo com seu sinal capturado e sua geometria, podemos executar as rotinas de Beamforming e deconvolução da imagem, e de reconstrução do sinal.

O trecho de código da listagem 6.3 importa o módulo que contém a classe Imager e em seguida instancia o objeto imager, recebendo o arranjo array que contém a configuração do arranjo e as medições feitas por seus microfones. A inicialização recebe também a frequência de interesse para a qual queremos olhar e a resolução do campo a ser reconstruído, no caso fieldRes $=32 \times 32$ é uma variável declarada na listagem 6.1.

O método Imager. beamform da forma que está exemplificado executa o algoritmo 
Listagem 6.3: Exemplo de uso da classe Imager

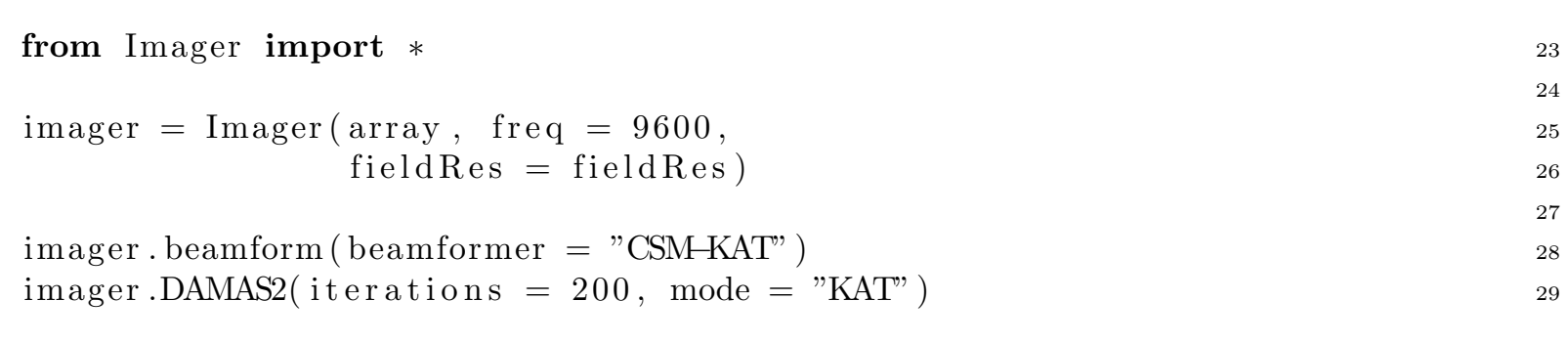

de beamforming CSM-KAT a partir matriz de correlação Cross-Spectral Matrix para a frequência especificada, e o algoritmo é acelerado pela transformada de Kronecker (KAT), como detalhado na seção 3.1.3. Outros algoritmos de beamformer foram implementados, notavelmente os métodos DAS e CSM-KAT retratados na seção 3.1.

O método Imager. DAMAS2 executa o algoritmo de deconvolução para a imagem criada pelo beamformer. O algoritmo KAT especificado utiliza a matriz A como referência para deconvolução e é acelerado pela KAT, detalhado em 3.2.3. Também foram implementados os modos PSF e A-matrix (Matriz A - sem aceleração), detalhados na seção 3.2.

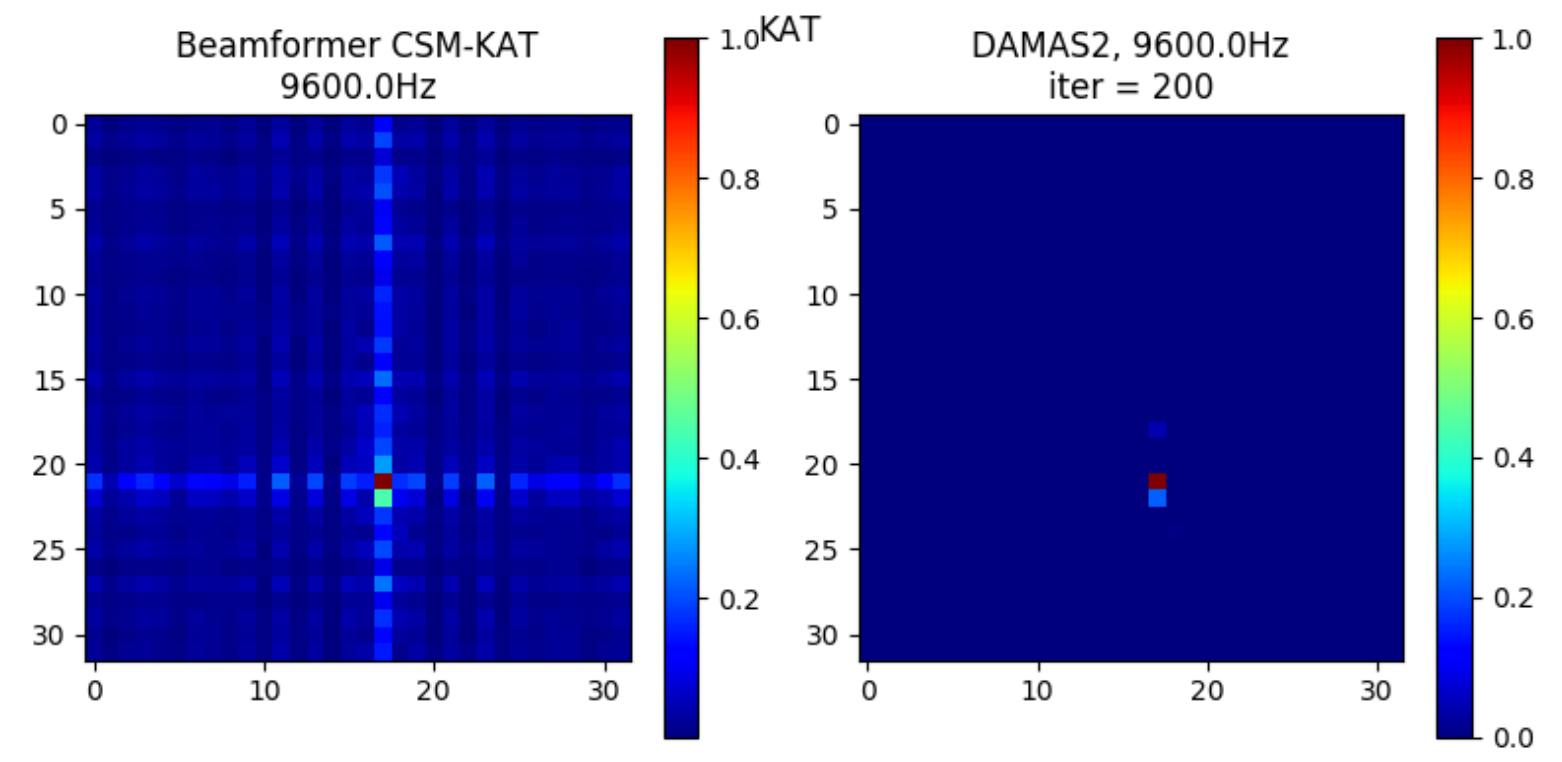

Figura 8: Resultados para beamformer e DAMAS2 calculados no exemplo.

A figura 8 nos permite comparar a imagem reconstruída por beamforming e a mesma imagem após deconvolução, utilizando 200 iterações do algoritmo DAMAS2.

A classe Imager também implementa funções de reconstrução de espectro de campo distante, estimando o sinal incidente estimando $\mathbf{y}(\omega)$ a partir de $\mathbf{x}(\omega)$ e $\mathbf{V}(\omega)$ segundo a equação (2.6). 


\subsubsection{SignalEstimator}

A classe SignalEstimator implementa a estimação de $\mathbf{y}$ para cada faixa de frequência da DFT, realiza a transformada inversa para o domínio do tempo e retorna uma estimação do sinal proveniente de uma direção de interesse.

Sua relação de herança com a classe Imager aproveita os métodos implementados para calcular variáveis como a matriz de direcionamento $\mathbf{V}$, além da implementação de alguns estimadores para $\mathbf{y}$ que varrem as faixas de frequência do janelamento utilizado.

O processamento realizado é flexível para permitir diferentes janelas no processamento, como retangular, Hann, Hanning, Blackman, além de funções de janelamento customizadas, com dimensões configuráveis. Outro artifício utilizado nas simulações foi utilização de zero-padding no janelamento para evitar problemas de aliasing temporal.

Parte dos métodos encapsulados nessa classe são equivalentes a métodos de imageamento que retornam o sinal y com amplitude e fase. Para métodos que retornam $y$, uma estimativa de intensidade, a recuperação já deixa de ser trivial. Alguns estimadores implementados são:

- Beamformers: delay-and-sum, delay-and-sum com aceleração via Kronecker array transform (KAT), minimum variance distortionless response (MVDR), minimum power distortionless response (MPDR);

- Métodos convexos: lasso, basis-pursuit (BP), basis-pursuit denoise (BPDN), total variation $(\mathrm{TV})$;

- Métodos gulosos: orthogonal matching pursuit (OMP), OMP com aceleração via KAT, L0-LS, L0-LS com aceleração via KAT;

- Outros: Mínimos quadrados.

Alguns métodos "híbridos" de reconstrução também foram implementados, como OMP progressivo (utilizando parte do suporte da estimativa para a última frequência como ponto de partida para a estimação da próxima), e DAMAS2/Mínimos quadrados (utilizando o suporte obtido através da deconvolução por DAMAS2 para estimar o sinal por mínimos quadrados). 


\subsection{Caracterização do sistema}

Através do simulador, podemos para um sistema composto por uma geometria de arranjo e uma discretização do espaço, calcular numericamente o parâmetro coerência descrito no capítulo 4. Usaremos a coerência como um indicador da qualidade de imagem ou reconstrução que o sistema projetado pode obter.

Como o nosso sistema é modelado pela equação (2.6)

$$
\mathbf{x}=\mathbf{V} \mathbf{y}
$$

a análise da matriz $\mathbf{V}$ poderá nos trazer informações sobre as possibilidades de se reconstruir o vetor $\mathbf{y}$ a partir do sinal recebido.

A estrutura da matriz V é pré-definida (2.4) (2.5), o que a torna uma função de 2 parâmetros: a geometria do arranjo (disposição dos sensores), e das direções definidas a se mapear. Podemos verificar como diferentes definições para esses parâmetros afetam a coerência (seção 4.2.2) da matriz V. Não analizaremos o spark (seção 4.2.1) pois sua natureza combinatória tem um custo computacional proibitivo, mesmo quando explorando as relações com o produto de Kronecker para facilitar o cálculo (4.8).

Para um dado campo a ser reconstruído, vamos comparar a coerência entre configurações de arranjo definidos na literatura. Iremos também variar a resolução do campo e verificar os efeitos em V. Para todos os testes utilizaremos uma distribuição uniforme de $u_{x}$ e $u_{y}$ entre $[-1,1]$, com o mesmo número de pixels em $x$ e $y$.

\subsubsection{Geometria}

A definição de coarranjo aqui apresentada é baseada em [20], com pequenas modificações. Com base no coarranjo são definidas geometrias tradicionais como de mínima redundância [21] e não-redundante [22]. Vamos considerar um arranjo linear com $N_{M i c}$ sensores de posições $\mathcal{D}=\left\{d_{1}, d_{2} \cdots d_{N_{M i c}}\right\}$. Cada posição $d_{i}$ é escolhida como um múltiplo inteiro de uma distância arbitrária denotada por $d_{0}$. Logo, dado $d_{0}$, podemos representar as posições por uma lista de inteiros $\overline{\mathcal{D}}=\left\{\bar{d}_{1}, \bar{d}_{2} \cdots \bar{d}_{N_{M i c}}\right\}$ onde $\bar{d}_{i}=\frac{d_{i}}{d_{0}}$.

O coarranjo desse arranjo será o conjunto das diferenças $\left|\hat{d}_{i}-\hat{d}_{j}\right|$, chamadas de espaçamento, para $i, j=1,2, \cdots, N_{M i c}, i \geq j$. Caso haja algum espaçamento inteiro ausente entre dois outros espaçamentos, consideramos que esse para espaçamento inteiro há um buraco no coarranjo. E caso haja algum espaçamento diferente de zero com mais 
de uma ocorrência, consideramos que esse espaçamento é redundante.

Os arranjos que compararemos nesta seção são arranjos separáveis de $8 \times 8$ microfones, com distribuição linear desses sensores nos eixos $x$ e $y$ iguais, detalhadas a seguir e ilustradas na figura 9. Isso, combinado com o fato de estarmos reconstruindo um campo com o mesmo número de direções em $x$ e $y$ e igualmente distribuídas conforme sugerido pelos resultados do capítulo 5 , nos permite decompor a matrix $\mathbf{V}$ do sistema em $\mathbf{V}=\mathbf{V}_{x} \otimes \mathbf{V}_{y}$ (2.8) onde $\mathbf{V}_{x}=\mathbf{V}_{y}$. Pela propriedade (4.13) podemos analisar a coerência de $\mathbf{V}_{x}$ ou $\mathbf{V}_{y}$ e estender os resultados. Para todos os casos nesta seção estamos considerando a reconstrução de um campo de $32 \times 32$ pixels.
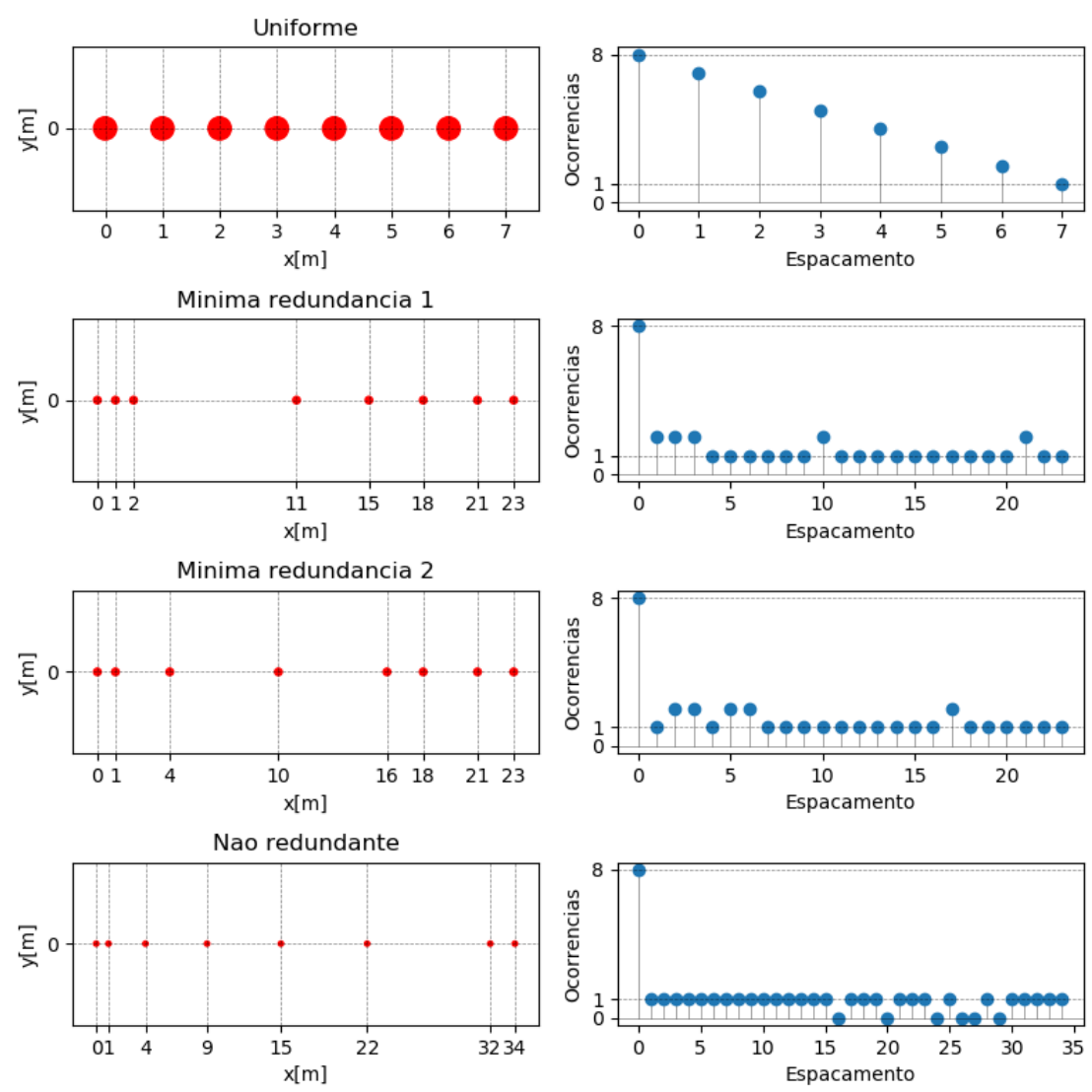

Figura 9: Posições $\overline{\mathcal{D}}$ de arranjos lineares de 8 microfones e seus respectivos coarranjos.

O arranjo uniforme é equi-espaçado e possui alta coerência para praticamente todas as frequências.

Os arranjos de mínima redundância foram inicialmente propostos em [21]. Um arranjo de mínima redundância é tal que seu coarranjo possui o maior número de espaçamentos distintos, com a restrição de que não haverá buracos no coarranjo.

Um arranjo não redundante [22] é aquele cujo coarranjo minimiza o número de buracos no coarranjo, com a restrição de não possuir espaçamentos redundantes (com mais de uma 
ocorrência). A obtenção das geometrias não-redundantes é feita combinatoriamente.

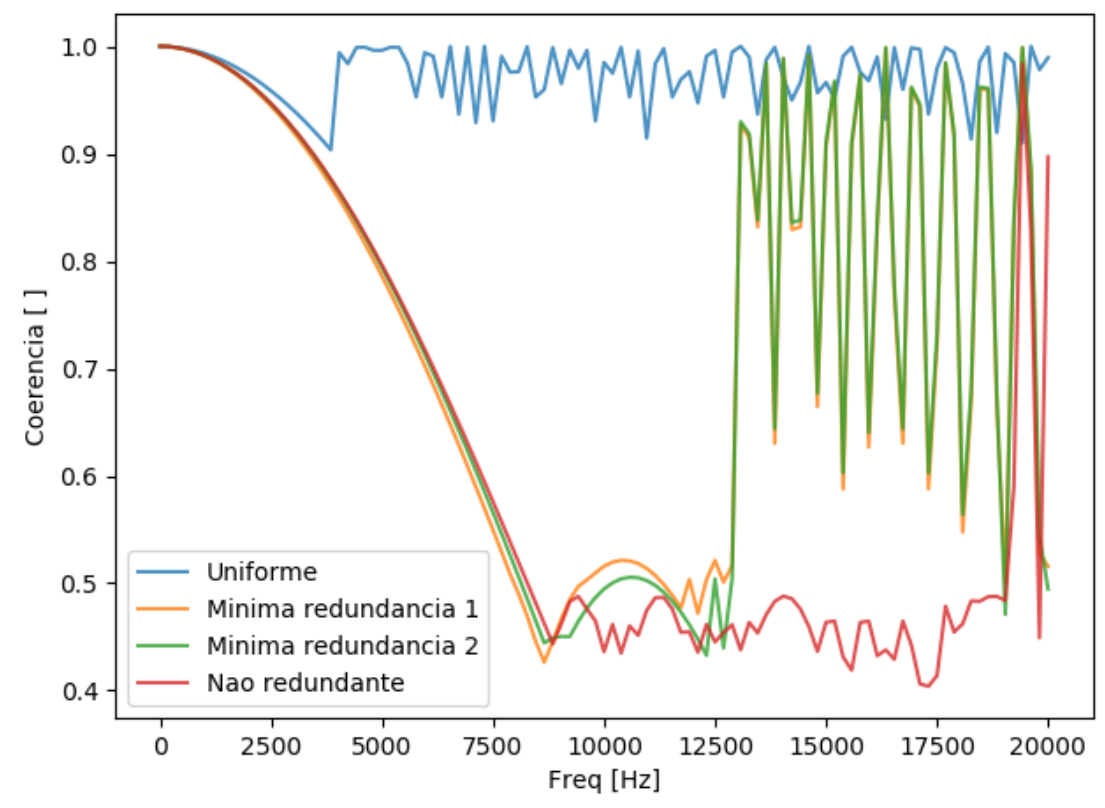

Figura 10: Comparação de coerência em função da frequência para diferentes geometrias.

Todas as configurações aqui apresentadas foram redimensionadas para as simulações para que a distância máxima entre 2 microfones em um mesmo eixo seja de $30 \mathrm{~cm}$.

Podemos ver na figura 10 que, entre as geometrias comparadas, a não-redundante apresenta menor coerência em uma faixa de frequências maior para arranjos com o mesmo número de microfones e mesma dimensão. Por esse parâmetro, entre essas geometrias clássicas comparadas, essa é a configuração com maior potencial para recuperação do sinal de campo distante através de reconstrução esparsa.

Ao tratar de reconstrução esparsa em arranjos lineares de sensores, [5] utiliza geometrias aleatórias de arranjos em seus experimentos e derivações. Espera-se que a coerência de uma geometria aleatória seja inferior à de um arranjo linear, mas não necessariamente será menor do que a de um arranjo determinístico, no caso o arranjo não redundante, como vemos na figura 11. Também está incluído no gráfico o arranjo aleatório com menor coerência na banda de interesse dentre os $10^{4}$ arranjos simulados.

\subsubsection{Resolução}

Uma das questões ao se projetar um arranjo é qual a resolução que se poderá obter com esse arranjo. Vimos no capítulo 5 que a discretização do espaço ideal para minimizar a coerência do sistema separável é uma distribuição uniforme de $u_{x}$ e $u_{y}$ entre $[-1,1]$, 


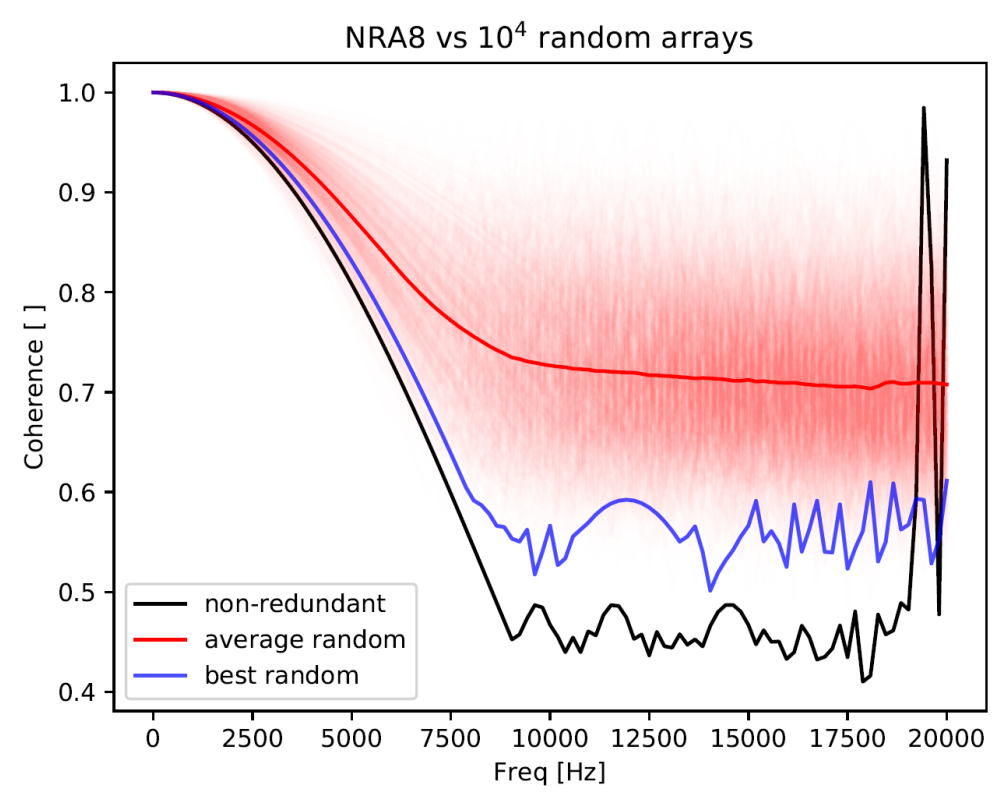

Figura 11: Coerência de $10^{4}$ arranjos aleatórios vs. não-redundante.

mas precisamos saber também qual o número de pixels que conseguimos reconstruir para então distribuí-los.

É esperado que a coerência seja mais elevada para um número maior de pixels, uma vez que estamos olhando a maior correlação entre diferentes colunas da matriz de direcionamento $\mathbf{V}$, e um número maior de direções implica num aumento no número de colunas de V. Seguindo também a linha de raciocínio utilizada na demonstração da distribuição ótima do espaço (capítulo 5), um número maior de direções u a serem distribuídas reduz o mínimo $\Delta u$ da discretização, elevando a coerência do sistema.

Aqui iremos comparar a coerência em um arranjo separável de $8 \times 8$ microfones para diferentes resoluções de campo distante. Utilizaremos para todos os casos a geometria não-redundante para a distribuição dos microfones.

A figura 12 ilustra a coerência em função da frequência para diferentes quantidades de direções na discretização uniforme do espaço. Ao aumentar a resolução, vemos que a coerência se eleva para baixas frequências, e passa a atingir um patamar de baixa coerência em frequências cada vez mais altas, até que ao atingir $20 \mathrm{kHz}$ ultrapassamos a banda do arranjo e a coerência volta a subir. Nesse caso, para dimensões superiores a $65 \times 65$ pixels a coerência não chegará a atingir o patamar inferior. 


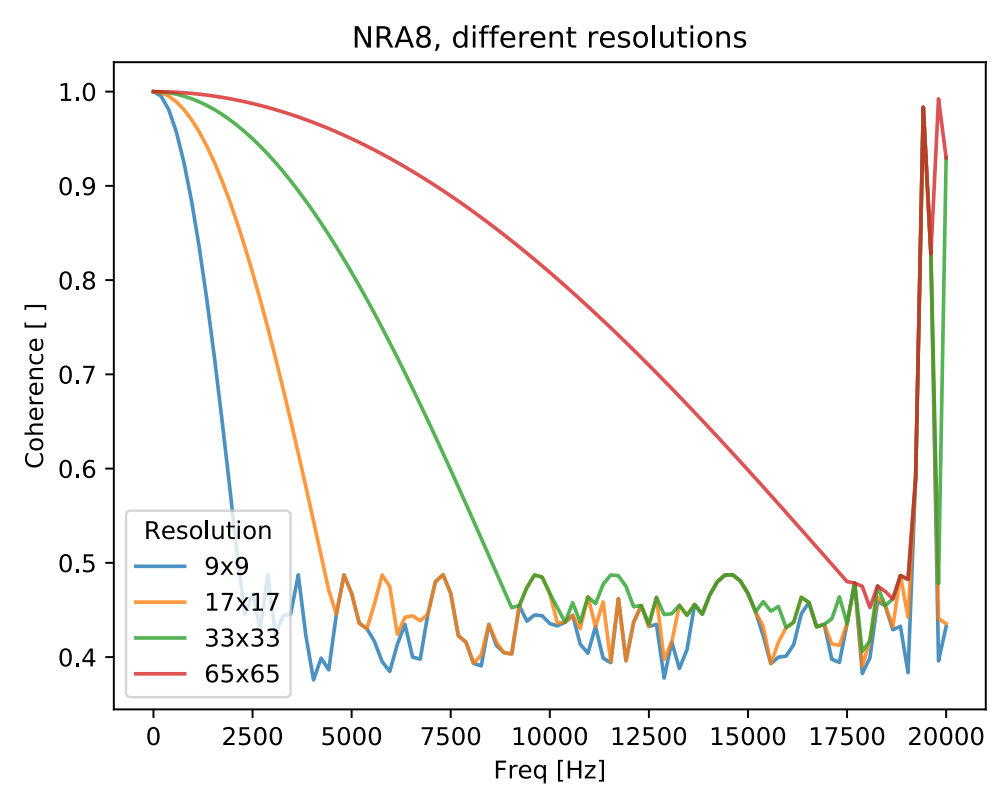

Figura 12: Comparação de coerência em função da frequência para diferentes resoluções.

\subsection{Imageamento e DOA}

Uma das razões de se gerar uma imagem acústica é identificar a direção de origem de um sinal incidente no arranjo, que é chamada de Direction of Arrival (DOA).

A partir da representação em imagem que recriamos do campo, podemos estimar as direções das fontes de chegada. Por exemplo, um campo com fontes sonoras em $\mathbf{u}_{s}=$ $[0,0,1]^{T}$ e $\mathbf{u}_{n}=\left[\frac{-1}{3}, \frac{-1}{3}, \frac{\sqrt{7}}{9}\right]^{T}$, com $N=1000$ amostras, com sinais senoidais na frequência de $10 \mathrm{kHz}$, é representado de maneira ideal na figura 13.

Através de algoritmos de beamforming (seção 3.1) podemos fazer estimativas iniciais do campo incidente utilizando o sinal capturado pelo arranjo. Como exitstirá uma pointspread function (PSF) para qualquer beamformer que utiliza a forma $\hat{\mathbf{y}}=\mathbf{W}^{H} \mathbf{x}$ (equação (3.3)) podemos tentar aprimorar a estimativa utilizando técnicas de deconvolução (seção 3.2). A figura 14 compara estimativas por beamforming DAS (seção 3.1.1), MVDR (seção 3.1.4), e MPDR (seção 3.1.5), e a progressão da deconvolução utilizando DAMAS2 por matriz A (seção 3.2.2) para cada um dos beamformers.

O imageamento também pode ser feito por técnicas de reconstrução esparsa (seção 4), que apresentam bons resultados, como pode ser visto na figura 15. O tempo de execução para cada método é detalhado na tabela 2.

Como o nosso sistema é decomponível em produto de Kronecker, a solução para a 
Tabela 2: Tempo de execução para algoritmos esparsos (30 iterações, média entre as 3 melhores)

\begin{tabular}{|lr|}
\hline Método & Tempo de Execução [s] \\
\hline \hline OMP & 0.011 \\
L0LS & 0.048 \\
Lasso & 3.590 \\
Basis-pursuit & 2.709 \\
BPDN & 2.631 \\
TV & 39.563 \\
\hline
\end{tabular}

regularização por Total Variation (seção 4.1.1) é colocada da forma

$$
\min _{\mathbf{x}}\left\{\|\nabla \mathbf{Y}\|_{2}: \mathbf{V}_{y} \mathbf{Y} \mathbf{V}_{x}^{T}=\mathbf{X}\right\}
$$

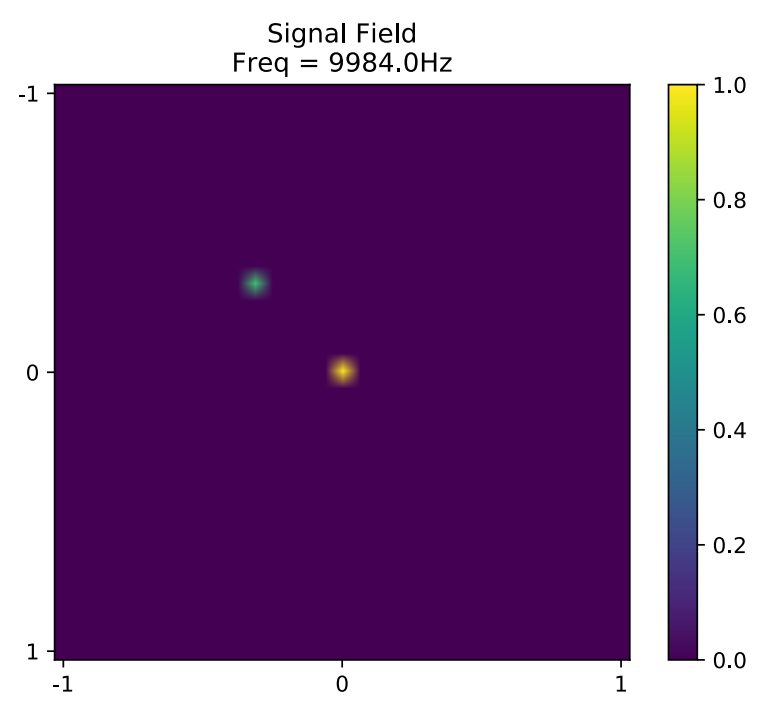

Figura 13: Campo de referência para exemplo com duas fontes. 

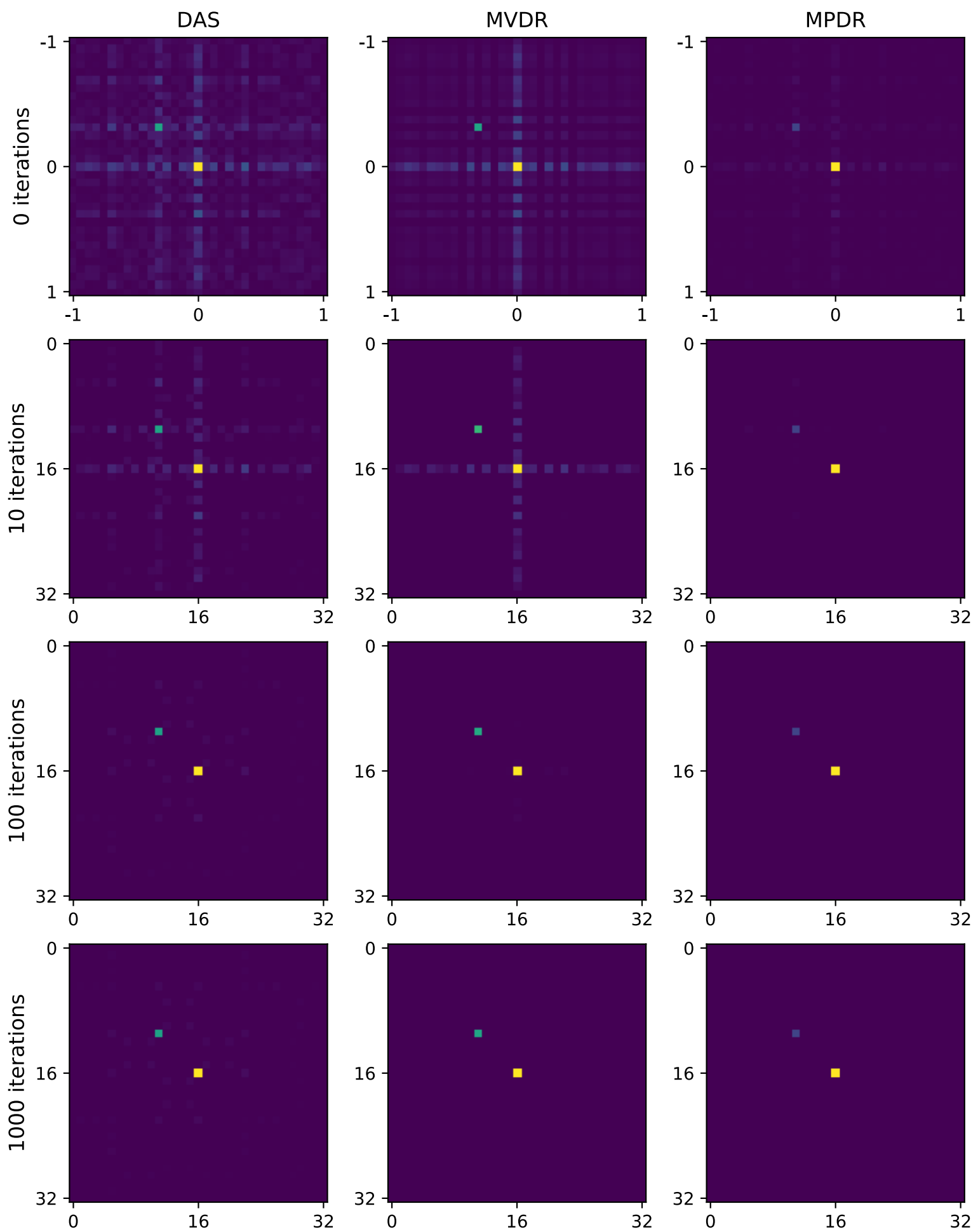

Figura 14: Reconstrução através de beamforming e deconvolução por matriz A. 

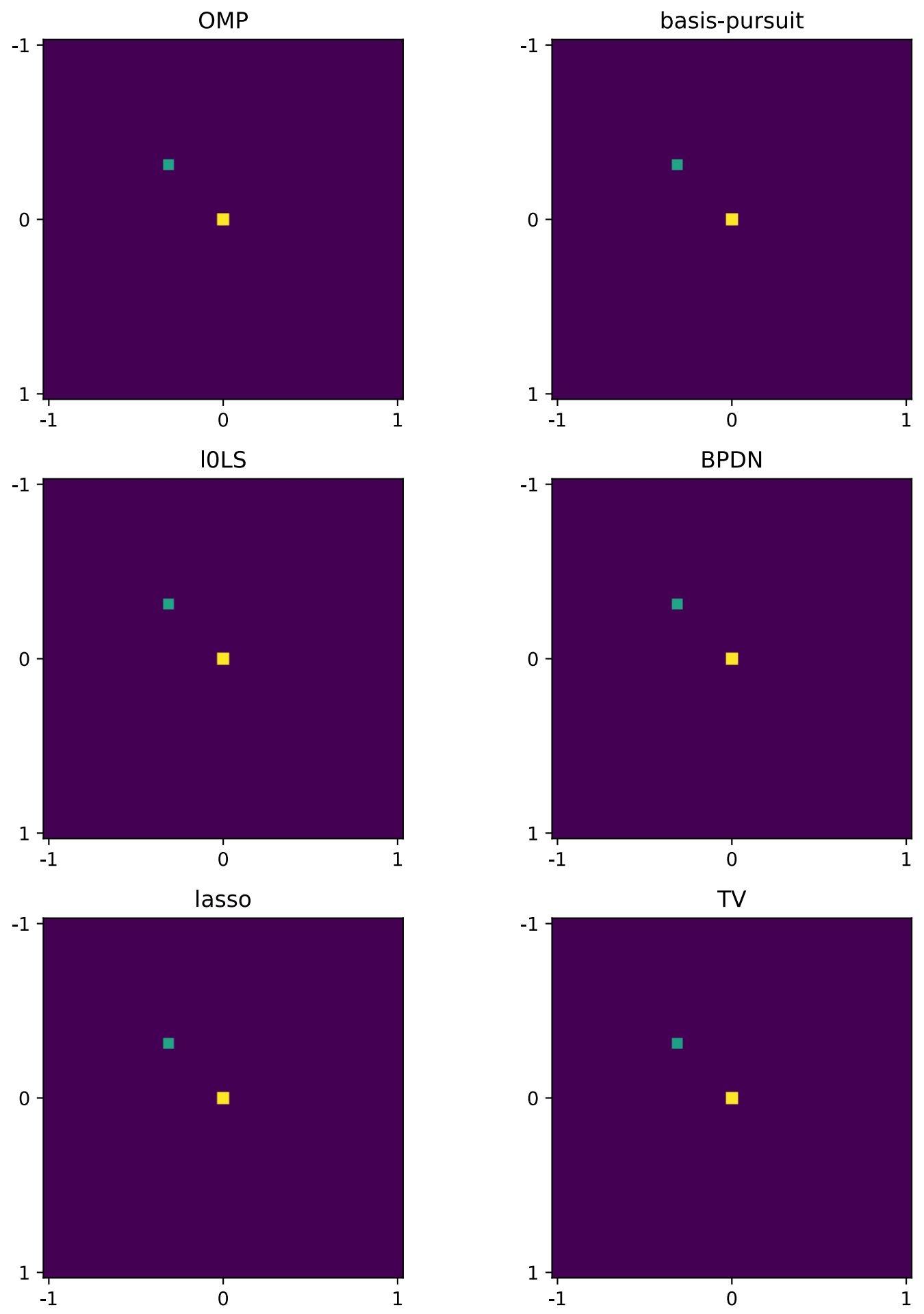

Figura 15: Reconstrução por algoritmos de reconstrução esparsa. 
O seguinte experimento foi elaborado para quantificar o erro de DOA. Vamos supor o caso em que queremos localizar uma fonte senoidal no caso em que há uma segunda fonte também senoidal na mesma frequência, tratada como interferência, incidindo da direção central $\mathbf{u}=[0,0,1]^{T}$. Para definir numericamente o erro de DOA, dada uma imagem reconstruída, pegaremos as direções das duas fontes de maior intensidade na reconstrução e calcularemos o ângulo entre a direção da fonte de interesse e cada uma das fontes estimadas. Consideraremos o menor valor como o erro de DOA.

As simulações de DOA foram realizadas com ruído de medida de $30 \mathrm{~dB}$ SNR, taxa de amostragem de $64 \mathrm{kHz}$, e FFT de uma única janela retangular com 100 pontos. Uma simulação foi feita em $16 \mathrm{kHz}$ (fig. 16), na faixa de baixa coerência para o arranjo de microfones, e outra para $2 \mathrm{kHz}$ (fig. 17), baixa frequência em que a coerência é mais alta. Foram simuladas $6 \times 10^{3}$ realizações e olharemos para a função de distribuição cumulativa do erro angular de localização $|\hat{\theta}-\theta|$.

Compararemos os erros de DOA entre os algoritmos Delay-and-Sum (DAS - seção 3.1.1), MVDR regularizado e OMP. Ao algoritmo MVDR é passada a informação da covariância do ruído, ou seja, ele possui mais informação prévia que os outros algoritmos. O OMP é configurado para 1\% de esparsidade máxima, e como condição de parada norma quadrática do erro $|\mathbf{x}-\mathbf{V} \hat{\mathbf{y}}|_{2} \leq 10^{-3}$.

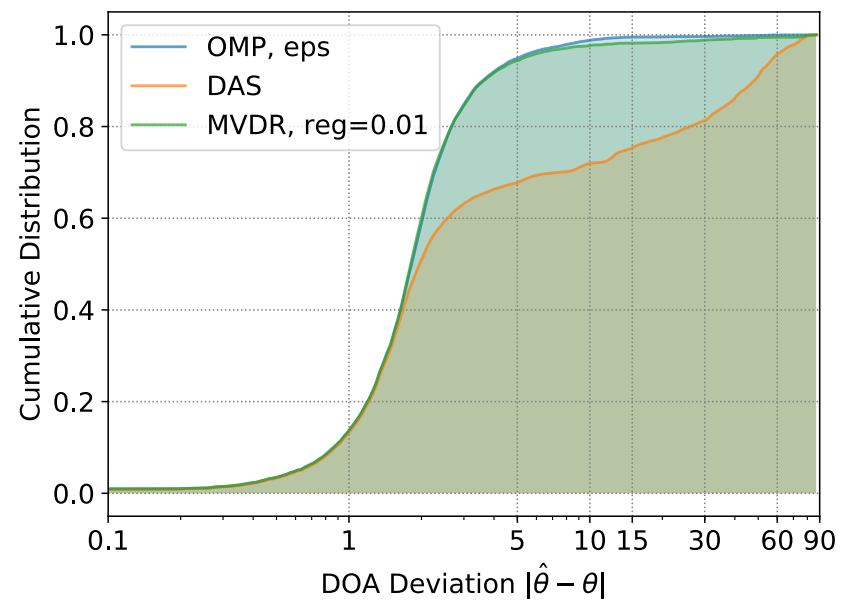

Figura 16: Erro de DOA para sinais na faixa de $16 \mathrm{kHz}$.

As direções escolhidas para o sinal foram aleatórias, mas, no caso excepcional em que todas as direções estão no grid, verificamos por simulações que o MVDR apresenta erro zero de DOA já que ele possui a informação total da covariância do ruído.

A partir das curvas de distribuição obtidas podemos concluir que, para a situação de duas fontes (de interesse e de ruído), o método que promove esparsidade (OMP) possui 


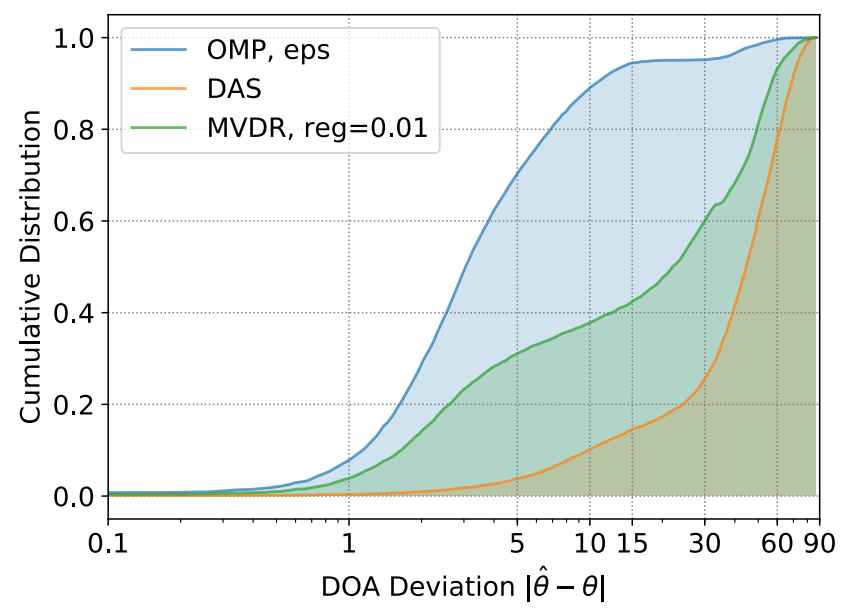

Figura 17: Erro de DOA para sinais na faixa de $2 \mathrm{kHz}$.

performance superior aos beamformers para estimação de DOA, especialmente em regiôes de alta coerência para o sistema. Ainda verificamos que, como esperado, a precisão do OMP é reduzida ao sair de uma frequência de baixa coerência para uma região de alta coerência. Apesar disso, os beamformers apresentaram uma perda muito maior, sendo mais evidente a vantagem de se utilizar o OMP nesse caso.

\subsection{Reconstrução do sinal no tempo}

Temos interesse em obter o sinal proveniente de uma direção específica. Para isso, estimamos o campo $\hat{\mathbf{y}}(\omega)$ varrendo todas as frequências discretas, e então realizamos a transformada inversa de Fourier do vetor obtido a partir dos coeficientes complexos de $\hat{y}\left(\omega_{0} \cdots \omega_{\max }: \mathbf{u}\right)$ para estimar o sinal no tempo vindo da direção $\mathbf{u}$.

Para reconstruir um sinal de comprimento indeterminado, o processaremos em janelas de duração constante que serão posteriormente reunidas. Para suprimir descontinuidades de borda entre as janelas, que causam artefatos sonoros de alta frequência como exemplificado na figura 18, utilizamos funções de janelamento com sobreposição entre as janelas, técnica conhecida como overlap-and-add. Utilizamos a transformada de Fourier de tempo curto (STFT) com janelamento Hanning, e propomos duas alternativas para reconstrução. Uma, realizando o overlap-and-add diretamente a partir da transformada inversa da janela (OLA) [23], com 50\% de sobreposição entre duas janelas consecutivas, e outra com o estimador de menor erro quadrático (LSEE), como recomendado em [24], em que o sinal obtido pela transformada de Fourier inversa da janela é novamente ponderado pela função de janelamento, utilizando $75 \%$ de sobreposição. 
Seja $x(n)$ o sinal de referência que queremos reconstruir, $X_{w}(k)$ o seu espectro obtido pós-janelamento de comprimento L, a partir do qual obtemos o espectro $Y(k)$ e sua transformada direta $y(n)$, e seja $w(n)$ a função de janelamento utilizada no janelamento de $x(n)$. A técnica LSEE estima uma sequência no tempo $\hat{x}(n)$ que minimiza o erro quadrático entre o espectro $X_{w}(k)$ do sinal de referência e $Y(k)$ da forma

$$
\hat{x}(n)=\frac{\sum_{m=-\infty}^{+\infty} w(m L-n) y(m L, n)}{\sum_{m=-\infty}^{+\infty} w^{2}(m L-n)} .
$$

Já a técnica OLA é expressada por

$$
\hat{x}(n)=\frac{\sum_{m=-\infty}^{+\infty} y(m L, n)}{\sum_{m=-\infty}^{+\infty} w(m L-n)} .
$$

Utilizar o LSEE exige computacionalmente algumas multiplicações a mais que a OLA, custo que perto do custo total da reconstrução de uma janela é desprezível. Para os valores de sobreposição utilizados, os denominadores são unitários. No entanto, pela sobreposição, para o LSEE precisamos reconstruir o dobro de janelas, o que traz um custo adicional para o ganho de desempenho que veremos nessa seção.

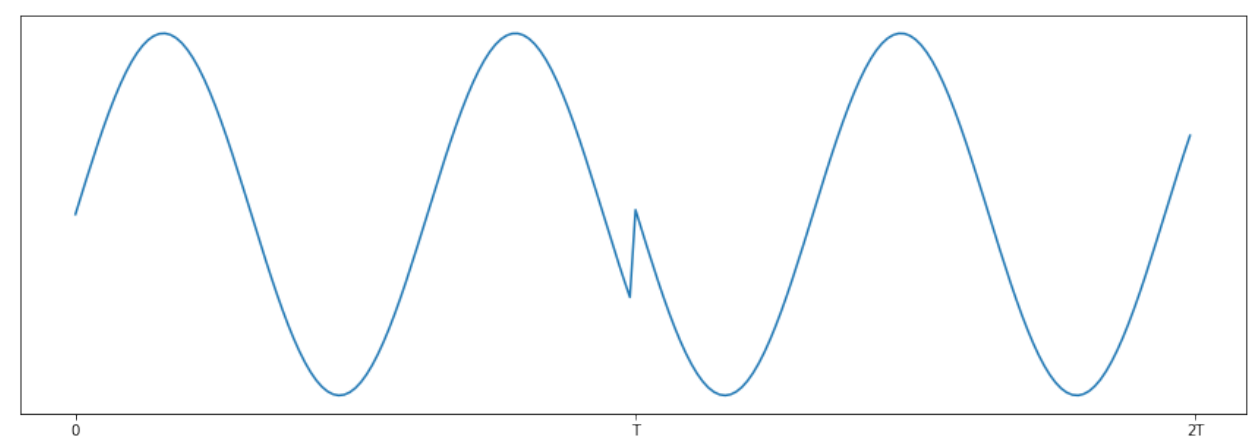

Figura 18: Exemplo de artefato causado pela discontinuidade entre duas janelas retangulares.

Um artefato imprevisto que foi identificado durante as simulações para reconstrução no tempo foi a presença de um eco circular dentro de cada janela da STFT, que para aplicações de estimação de DOA seria irrelevante por se tratar de uma distorção de fase. Esse tipo de distorção é característica de convoluções circulares, e é esperada em operações lineares de multiplicação no domínio da frequência. No entanto, estamos tratando cada banda individualmente e, no caso do OMP, o processamento é também não-linear. Apesar disso, tratamos o efeito de aliasing no tempo com uma solução utilizada para mitigar a convolução circular. Como sugerido em [23], dobramos o tamanho da janela de sinal selecionada de tamanho $N$ e adicionamos $N$ amostras nulas no final, prática conhecida como zero-padding. Ao fazer a transformada inversa, selecionamos apenas as $N$ primeiras 
amostras. Essa prática também pode ser interpretada como uma sobreamostragem na frequência ao realizarmos a transformada de Fourier.

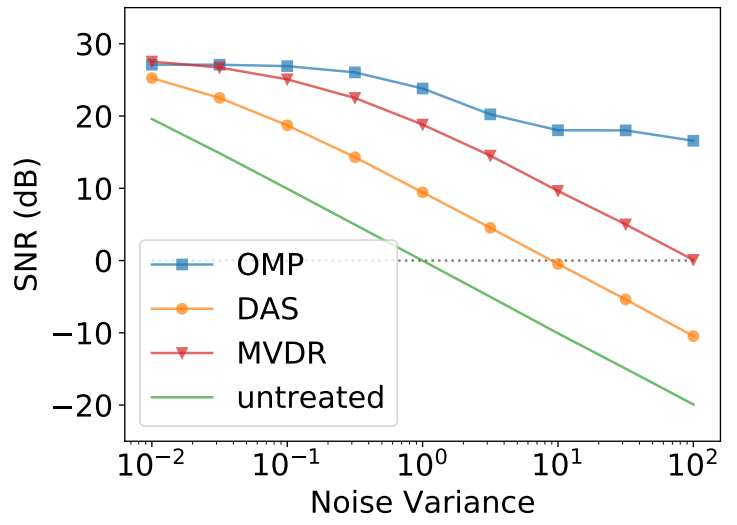

(a) Reconstrução OLA

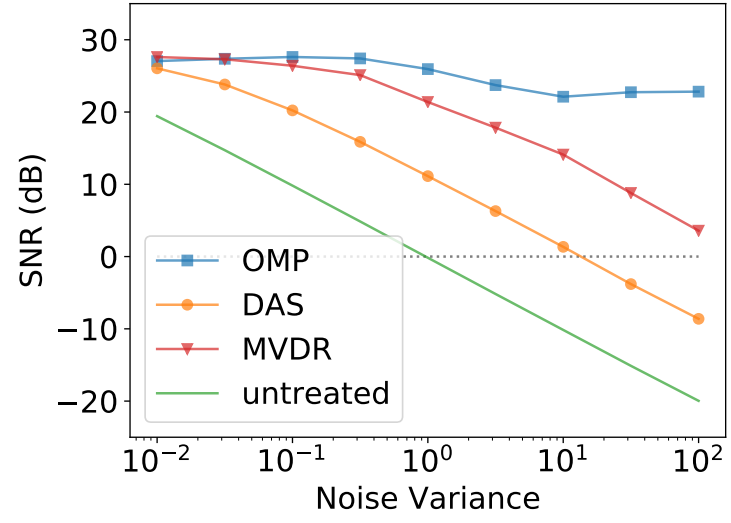

(b) Reconstrução LSEE

Figura 19: Caso 1, sinal de banda $11 \mathrm{kHz}-17 \mathrm{kHz}$.

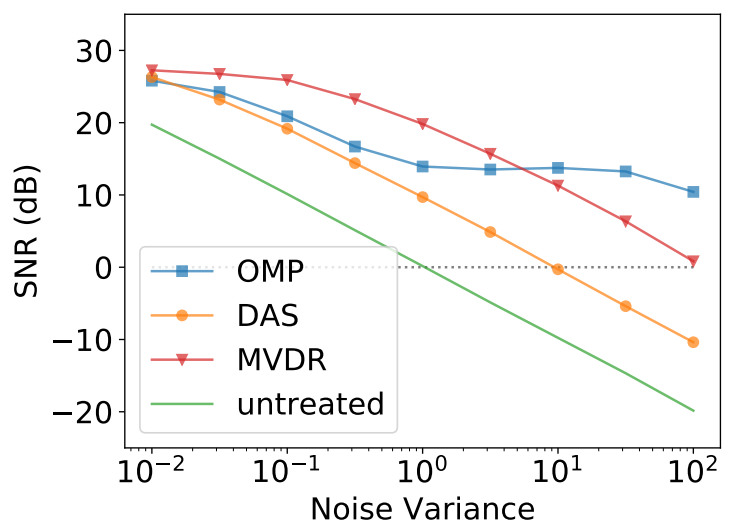

(a) Reconstrução OLA

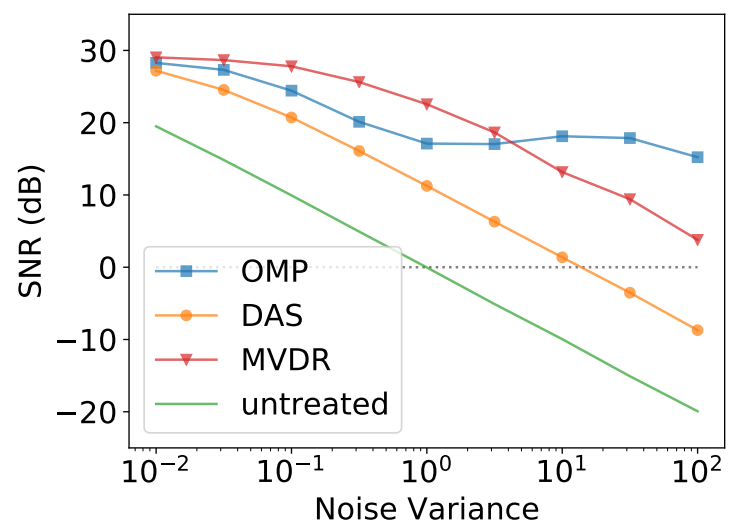

(b) Reconstrução LSEE

Figura 20: Caso 2, sinal de banda $2 \mathrm{kHz}-17 \mathrm{kHz}$.

O experimento elaborado tem o objetivo de comparar os resultados de reconstrução obtidos através da estimação esparsa do OMP (seção 4.1.2.1) e pelos beamformers DAS (seção 3.1.1) e MVDR (seção 3.1.4). Os algoritmos seguem a mesma configuração da seção 6.3 .

$\mathrm{Na}$ simulação, queremos reconstruir o sinal proveniente da direção central $\mathbf{u}_{s}=$ $\left[\begin{array}{lll}0 & 0 & 1\end{array}\right]^{T}$, enquanto há uma fonte pontual de interferência na direção $\mathbf{u}_{n}=\left[\begin{array}{ll}0 \frac{-1}{2} & \frac{\sqrt{3}}{2}\end{array}\right]^{T}$. Ruído Gaussiano independente é adicionado a cada microfone para criar ruído de medida de magnitude $30 \mathrm{~dB}$.

Em um primeiro caso, o sinal de interesse é ruído branco filtrado de banda $11 \mathrm{kHz}-$ $17 \mathrm{kHz}$, e para um segundo temos ruído branco de banda $2 \mathrm{kHz}-17 \mathrm{kHz}$. Em ambos os 
casos, a fonte de interferência é ruído branco filtrado de banda $0 \mathrm{kHz}-18 \mathrm{kHz}$. Para cada caso estamos apresentando a média de 150 realizações.

A relação sinal-ruído (SNR) entre o sinal de referência e o sinal reconstruído está ilustrada nas figuras 19 e 20. O OMP trouxe bons resultados em situações de alto ruído, especialmente quando trabalhando na banda de frequências de menor coerência para o arranjo. Ao utilizar técnicas que forçam esparsidade na solução, eliminamos lóbulos principais e laterais, reduzindo a interferência entre fontes.

Para um mesmo algoritmo, comparamos os desempenhos entre as técnicas de reconstrução OLA e LSEE, com os resultados de redução de ruído ilustrados na figura 21. Podemos ver que, para os casos analisados, o LSEE traz um ganho de até $6 \mathrm{~dB}$ em relação ao OLA, mas atrelado a aproximadamente o dobro do custo computacional.

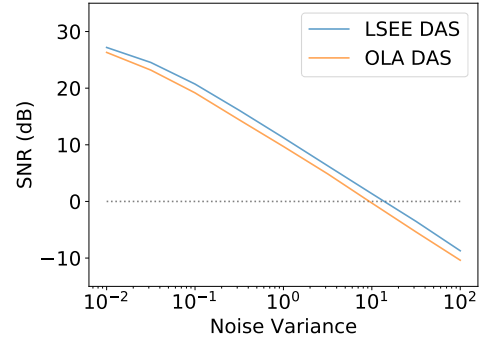

(a) DAS, $2 \mathrm{kHz}-17 \mathrm{kHz}$

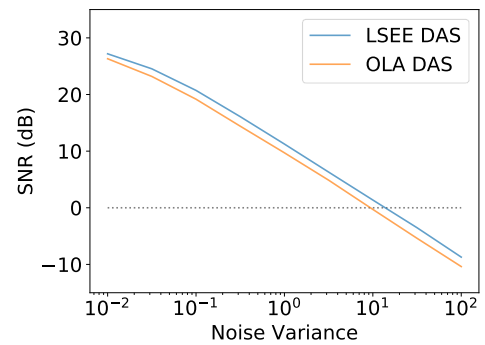

(d) DAS, $11 \mathrm{kHz}-17 \mathrm{kHz}$

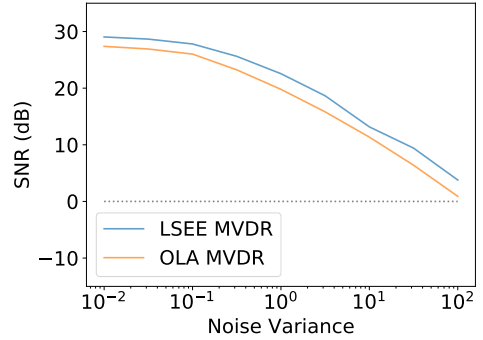

(b) MCDR, $2 \mathrm{kHz}-17 \mathrm{kHz}$

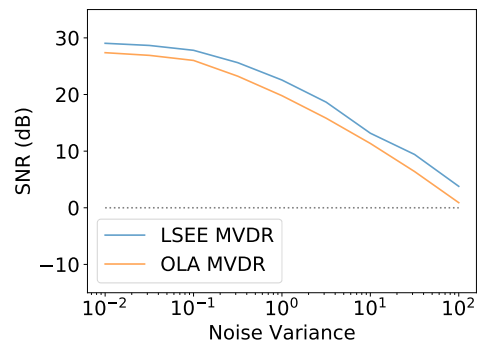

(e) MVDR, $11 \mathrm{kHz}-17 \mathrm{kHz}$

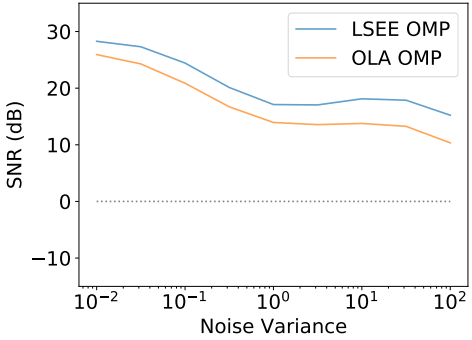

(c) OMP, $2 \mathrm{kHz}-17 \mathrm{kHz}$

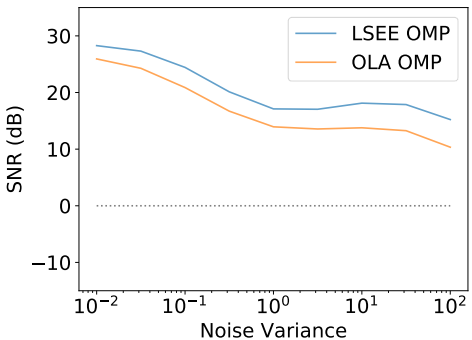

(f) $\mathrm{OMP}, 11 \mathrm{kHz}-17 \mathrm{kHz}$

Figura 21: Desempenho do janelamento OLA vs LSEE.

Para o OMP, o método de reconstruir cada banda de frequência individualmente e realizar a transformada inversa é a maneira mais simples para se recuperar o sinal no tempo. O método pode ser elaborado de algumas maneiras, como utilizar um ponto de partida não nulo para o suporte, o que reduz a complexidade do algoritmo e permite a prédeterminação de uma direção a ser reconstruída. Podemos fazer isso caso o DOA seja préestabelecido, ou o habilitando repasse de informação entre bandas de frequência. Vemos então os resultados do OMP obtidos aqui como um ponto de partida para aprimorações futuras. 


\section{CONCLUSÃO}

Este trabalho discorre sobre como qualificar objetivamente a geometria de um arranjo, tomando como base a sua medida de coerência, e compara a abordagem tradicional de beamforming por filtragem espacial com uma abordagem do ponto de vista de reconstrução esparsa.

As geometrias analisadas pertencem à classe de arranjos bidimensionais separáveis, em que aceleração computacional através da KAT é aplicável. O cálculo da coerência para sistemas separáveis também é facilitado pela KAT, e na seção 6.2 comparamos geometrias determinísticas tradicionais, e a geometria de arranjo não redundante com geometrias aleatórias, utilizadas na literatura para análise de beamforming por reconstrução esparsa.

A análise da coerência do arranjo em função da frequência nos permite encontrar a banda de melhor performance para uma geometria. Em contrapartida, podemos escolher uma geometria que melhor atenda as necessidades de frequência, atuando como uma ferramenta de projeto. Ainda resta analisar outras garantias de reconstrução para o sistema, como spark (seção 4.2.1) ou restricted isometry property (RIP) [25], que exigem cálculos muito mais custosos que o cálculo da coerência para uma matriz.

A discretização uniforme do espaço já era utilizada para melhorar a aproximação da deconvolução utilizando a point-spread function (PSF) (seção 3.2.1). No capítulo 5 também mostramos que essa discretização minimiza a coerência do sistema, tornando-a uma boa recomendação quando utilizamos técnicas de reconstrução esparsa.

Para o problema de localização de fontes (DOA), além dos algoritmos de beamforming DAS, MVDR, e MPDR, aos quais podem ser aplicadas técnicas de deconvolução, foram implementados outros algoritmos de reconstrução esparsa, com exemplo de imageamento na seção 6.3. Dentre esses algoritmos, o OMP (seção 4.1.2.1) foi utilizado para comparação por apresentar bons resultados e performance computacional.

Ao determinar a direção de chegada, o OMP apresentou desempenho semelhante ou superior ao MVDR, recebendo as mesmas informações do DAS - sem imformação prévia 
sobre a covariância do ruído. Para reconstrução de sinais no tempo, o OMP também apresenta bom desempenho, superior ao MVDR em situações de maior ruído ou para frequências de baixa coerência para o arranjo.

A implementação de imageamento acústico em tempo real já ocorre por filtragem espacial em sistemas para detecção de vazamento de gás [26]. A redução de complexidade computacional e de memória trazida pela KAT ao arranjo separável pode tornar viável a implementação de algoritmos de melhor desempenho a sistemas de tempo real e embarcados.

Alguns tópicos que essa dissertação não cobre mas que podem ser explorados futuramente:

- Comparação entre entre algoritmos esparsos para erro angular de localização (DOA) e redução de ruído na reconstrução direcional de sinal;

- Comparação de geometrias utilizando como parâmetro outras garantias de reconstrução;

- Analisar a performance de algoritmos híbridos de reconstrução no tempo, tais como gerar o suporte através de DAMAS2 e estimar os fatores complexos por mínimos quadrados, compartilhamento de suporte entre frequências vizinhas na FFT, etc. 


\section{REFERÊNCIAS}

1 TREES, H. V. Optimum Array Processing: Part IV of Detection, Estimation, and Modulation Theory. [S.l.]: Wiley, 2004. (Detection, Estimation, and Modulation Theory). ISBN 9780471463832.

2 DOUGHERTY, R. Extensions of DAMAS and benefits and limitations of deconvolution in beamforming. 11th AIAA/CEAS Aeroacoustics Conference, 2005.

3 YARDIBI, T. et al. Sparsity constrained deconvolution approaches for acoustic source mapping. The Journal of the Acoustical Society of America, v. 123, p. 2631, 2008.

4 MASIERO, B.; NASCIMENTO, V. H. Revisiting the Kronecker array transform. IEEE Signal Processing Letters, v. 24, n. 5, p. 525-529, maio 2017. ISSN 1070-9908.

5 XENAKI, A.; GERSTOFT, P.; MOSEGAARD, K. Compressive beamforming. The Journal of the Acoustical Society of America, v. 136, p. 260, 072014.

6 DAHER, M. A. Acoustic simulation software for separable microphone arrays. [S.1.]: GitHub, 2019. 〈https://github.com/marcelodaher/ArraySim〉.

7 DAHER, M. A. et al. Compressive beamforming and directional sound reconstruction using the kronecker array transform. Proceedings of 53rd Asilomar Conference on Signals, Systems and Computers, 2019.

8 RIBEIRO, F. P.; NASCIMENTO, V. H. Fast transforms for acoustic imaging - part

I: Theory. IEEE Transactions on Image Processing, v. 20, n. 8, p. 2229-2240, 2011.

9 BREWER, J. Kronecker products and matrix calculus in system theory. IEEE Transactions on Circuits and Systems, v. 25, n. 9, p. 772-781, Sep 1978. ISSN 0098-4094.

10 RIBEIRO, F. P. Arrays de microfones para medida de campos acusticos. Tese (Doutorado) - Escola Politecnica, Universidade de Sao Paulo, 2012.

11 NASCIMENTO, V. H.; MASIERO, B. S.; RIBEIRO, F. P. Acoustic imaging using the Kronecker array transform. In: COELHO, R. F. et al. (Ed.). Signals and Images: Advances and Results in Speech, Estimation, Compression, Recognition, Filtering, and Processing. [S.l.]: CRC Press, 2015. cap. 6, p. 153-178.

12 LEV-ARI, H. Efficient solution of linear matrix equations with application to multistatic antenna array processing. Commun. Inf. Syst., International Press of Boston, v. 05, n. 1, p. 123-130, 2005. Disponível em: 〈https://projecteuclid.org: 443/euclid.cis/1149698475>.

13 LI, J.; STOICA, P.; WANG, Z. On robust capon beamforming and diagonal loading. In: 2003 IEEE International Conference on Acoustics, Speech, and Signal Processing, 2003. Proceedings. (ICASSP '03). [S.l.: s.n.], 2003. v. 5, p. V-337. ISSN 1520-6149. 
14 BARANIUK, R. et al. An Introduction to Compressive Sensing. Disponível em: $\langle$ https://legacy.cnx.org/content/col11133/1.5/〉.

15 STURM, B. L.; CHRISTEnSEN, M. G. Comparison of orthogonal matching pursuit implementations. In: 2012 Proceedings of the 20th European Signal Processing Conference (EUSIPCO). [S.l.: s.n.], 2012. p. 220-224. ISSN 2219-5491.

16 DONOHO, D. L.; ELAD, M. Optimally sparse representation in general (nonorthogonal) dictionaries via 11 minimization. Proceedings of the National Academy of Sciences, National Academy of Sciences, v. 100, n. 5, p. 2197-2202, 2003. ISSN 0027-8424. Disponível em:〈http://www.pnas.org/content/100/5/2197〉.

17 JOKAR, S.; MEHRMANN, V. Sparse solutions to underdetermined Kronecker product systems. Linear Algebra and its Applications, v. 431, n. 12, p. $2437-2447$, 2009. ISSN 0024-3795. Special Issue in honor of Shmuel Friedland. Disponível em: 〈http://www.sciencedirect.com/science/article/pii/S0024379509004066〉.

18 GRIBONVAL, R.; NIELSEN, M. Sparse representations in unions of bases. IEEE Transactions on Information Theory, v. 49, n. 12, p. 3320-3325, Dec 2003. ISSN 0018-9448.

19 NUMPY.FFT.RFFTFREQ. Disponível em: 〈https://docs.scipy.org/doc/numpy-1. 13.0/reference/generated/numpy.fft.rfftfreq.html〉.

20 WANG, M.; NEHORAI, A. Coarrays, music, and the cramér-rao bound. IEEE Transactions on Signal Processing, v. 65, n. 4, p. 933-946, Feb 2017. ISSN 1053-587X.

21 MOFFET, A. Minimum-redundancy linear arrays. IEEE Transactions on Antennas and Propagation, v. 16, n. 2, p. 172-175, Mar 1968. ISSN 0018-926X.

22 VERTATSCHITSCH, E.; HAYKIN, S. Nonredundant arrays. Proceedings of the IEEE, v. 74, n. 1, p. 217-217, Jan 1986. ISSN 0018-9219.

23 ALLEN, J. Short term spectral analysis, synthesis, and modification by discrete fourier transform. IEEE Transactions on Acoustics, Speech, and Signal Processing, v. 25, n. 3, p. 235-238, June 1977. ISSN 0096-3518.

24 Griffin, D. Signal estimation from modified short-time Fourier transform. and Signal Processing IEEE Transactions on Acoustics, Speech, v. 32, n. 2, p. 236-243, abr. 1984. ISSN 0096-3518.

25 Needell, D.; Vershynin, R. Signal recovery from incomplete and inaccurate measurements via regularized orthogonal matching pursuit. IEEE Journal of Selected Topics in Signal Processing, v. 4, n. 2, p. 310-316, April 2010. ISSN 1932-4553.

26 Fluke Industrial. Ultrasonic Leak Detector - ii900 Sonic Industrial Imager. Disponível em: 〈https://www.fluke.com/en/product/industrial-imaging/ sonic-industrial-imager-ii900 $\rangle$. 
ANEXO A - PUBLICAÇÕES RELEVANTES 


\title{
Compressive beamforming and directional sound reconstruction using the Kronecker array transform
}

\author{
Marcelo A. Daher, Carlos A. Prete Jr., Vítor H. Nascimento \\ Dept. of Electronic Systems Engineering \\ University of São Paulo \\ São Paulo, Brazil \\ \{marcelo.daher,carlos.prete,vitnasci\}@usp.br
}

\author{
Bruno S. Masiero \\ Dept. of Communications \\ University of Campinas \\ Campinas, Brazil \\ masiero@unicamp.br
}

\begin{abstract}
The Kronecker array transform (KAT) was introduced to reduce the computational burden in acoustic image duced to reduce the computational burden in acoustic image
estimation and other 2-dimensional array processing applications. The KAT can be applied only when the planar microphone array is separable. In this paper we study the performance of separable arrays using compressive beamforming algorithms to estimate the direction of arrival (DOA) of far-field sources and to recover the signal arriving from a particular direction. Our analysis extends previous studies based on mutual coherence to an lo separable arrays, and shows that non-redundant separable arrays have better mutual coherence than can be obtained using 2D random arrays. We also derive the space discretization that yields the minimum coherence, and study the influence of frequency and the spatial resolution on the coherence. In order to verify the results, we present the performance of a separable array in two problems: DOA estimation and signal recovery using sparse reconstruction, and compare its performance with classical sparse better performance in DOA estimation and great improvement better performance in DOA estim
in time-domain signal recovery.

in time-domain signal recovery. ration, signal denoising, compressed sensing.
\end{abstract}

\section{INTRODUCTION}

The Kronecker array transform (KAT) was introduced with the goal of reducing computations in algorithms for acoustic image estimation under the condition that the microphone array is designed with a separable geometry [1], [2]. The KAT can be applied to a number of different acoustic imaging algorithms [2], such as conventional beamforming [3], DAMAS [4], and covariance-fitting [5]. Moreover, the KAT has been recently extended to more general beamforming applications in [6]. And although the use of regularized optimization [5] and sparse estimation techniques [2] has been proposed in order to achieve better resolution of the acoustic images, using either $\ell_{1}$ or total variation regularization, the performance of the accelerated algorithms was not studied from a compressive sensing point of view.

Application of compressive sensing ideas to beamforming has been proposed in [7], [8] and, more recently, Xenaki et al. [9] studied the performance of direction-of-arrival (DOA) algorithms based on compressive sensing, considering onedimensional random arrays, relating performance measures (such as resolution) to the coherence of the measurement matrix. The performance of nonrandom arrays was considered in [10], which used an extension of the restricted isometry condition (RIP) to find the probability that a set of $k$-sparse source directions will be recovered.

In this paper we study the performance of separable arrays in recovering the directions, intensities and the actual timesignals of a small number of sound sources impinging on a two-dimensional array. Our analysis is based on the coherence of the measurement matrix (following [9]), but extending previous results in four ways: (i) we consider two-dimensional arrays, (ii) we consider separable arrays for which the KAT can be applied, (iii) we evaluate the performance as a function of the signal frequency, and (iv) we evaluate the recovery of the time signals. A further contribution is our analysis of the best way to discretize the look directions. Although there are methods to improve the resolution of compressive-sensing beamforming techniques, using grid refinement or the atomic norm [11], our analysis is restricted in this paper to a fixed, finite number of look directions. In addition, although there are source localization methods for broadband signals that consider the full spectrum of the signal [12], we show here that, in the case of a small number of sources, processing each frequency independently already gives good results for the reconstruction of the time signal coming from a particular direction.

The remainder of this paper is organized as follows. In Section II we describe the signal model, while Section III describes the assumptions on the array and look directions for application of the Kronecker array transform. Section IV compares the coherence of measurement matrices obtained from different choices of arrays as a function of the signal frequency, and Section V finds the space discretization that results in minimal coherence. Section VI shows the performance of the chosen separable array with DOA estimation, Section VII considers the problem of signal recovery, and Section VIII concludes the paper.

\section{Problem Overview}

Consider a microphone array with $N_{\text {Mic }}$ microphones, receiving signals from sources in the far field, discretized in $N_{\text {Dir }}$ directions of interest. Each direction is represented by a unitary vector $\mathbf{u}$, perpendicular to the plane wave traveling from this 
direction. The vector $\mathbf{u}$ is defined in spherical coordinates in terms of the polar angle $\theta$ and the azimuthal angle $\phi$ as

$$
\mathbf{u}=\left[\begin{array}{c}
u_{x} \\
u_{y} \\
\sqrt{1-u_{x}^{2}-u_{y}^{2}}
\end{array}\right]=\left[\begin{array}{c}
\sin \theta \cos \phi \\
\sin \theta \sin \phi \\
\cos \theta
\end{array}\right] .
$$

Given a signal coming from the direction $\mathbf{u}$ and the position $\mathbf{p}_{i}$ of the $i$-th microphone, we can calculate the time delay $\tau_{i}$, with respect to the origin, for the signal to reach each microphone, as $\tau_{i}=-\frac{\mathbf{u}^{T} \mathbf{p}_{i}}{c}$, where $c$ is the speed of sound in the medium.

For a given direction $\mathbf{u}$ and an angular frequency $\omega$, we define a delay vector $\mathbf{v}(\omega: \mathbf{u})$ containing the frequency delays of a given direction for the $N_{\text {Mic }}$ microphones in our array

$$
\mathbf{v}(\omega: \mathbf{u})=\left[\begin{array}{c}
e^{j \omega \tau_{0}} \\
e^{j \omega \tau_{1}} \\
\vdots \\
e^{j \omega \tau_{\left(N_{\mathrm{Mic}}-1\right)}}
\end{array}\right]=\left[\begin{array}{c}
e^{-j \omega \mathbf{u}^{T} \mathbf{p}_{0} / c} \\
e^{-j \omega \mathbf{u}^{T} \mathbf{p}_{1} / c} \\
\vdots \\
e^{-j \omega \mathbf{u}^{T} \mathbf{p}_{\left(N_{\mathrm{Mic}}-1\right)} / c}
\end{array}\right] .
$$

Once the $N_{\text {Dir }}$ directions we will look at are chosen, we define an $\left[N_{\text {Mic }} \times N_{\text {Dir }}\right]$ matrix

$$
\mathbf{V}(\omega)=\left[\mathbf{v}\left(\omega: \mathbf{u}_{0}\right) ; \mathbf{v}\left(\omega: \mathbf{u}_{1}\right) ; \cdots ; \mathbf{v}\left(\omega: \mathbf{u}_{\left(N_{\text {Dir }}-1\right)}\right)\right]
$$

We also define the vector $\mathbf{x}(\omega)$ containing the windowed Discrete Fourier Transforms of the signal from all microphones for a frequency $\omega=k 2 \pi / L, 0 \leq k \leq L-1$, where $L$ is the DFT length, and the vector $\mathbf{y}(\bar{\omega})$ containing the far field signals arriving from each direction:

$$
\begin{aligned}
& \mathbf{x}(\omega)=\left[x_{0}(\omega), x_{1}(\omega), \cdots, x_{N_{\text {Mic }}-1}(\omega)\right]^{T}, \\
& \mathbf{y}(\omega)=\left[y_{0}(\omega), y_{1}(\omega), \cdots, y_{N \text { dir }-1}(\omega)\right]^{T} .
\end{aligned}
$$

In our model, the signal vector $\mathbf{x}(\omega)$ received by the microphone array is the sum of the $\mathbf{y}(\omega)$ signals with the correspondent delays applied for each microphone, thus

$$
\mathbf{x}(\omega)=\mathbf{V}(\omega) \cdot \mathbf{y}(\omega)
$$

Our goal is to recover $\mathbf{y}(\omega)$ from $\mathbf{x}(\omega)$ and $\mathbf{V}(\omega)$, which is an ill-posed problem. We compare the quality of the estimates $\hat{\mathbf{y}}(\omega)$ obtained for all frequencies of interest by sparse methods with the Delay-and-Sum (DAS) beamformer and the Minimum Variance Distortionless Response (MVDR) beamformer [13], as well as the reconstructions of the time signals $y_{k}(t), k=0, \ldots, N_{\text {dir }}-1$.

\section{Separable Arrays}

When working with a 2-dimensional microphone array, we can design our system in a specific way to allow the decomposition of the matrix $\mathbf{V}$ as a Kronecker product of two other matrices, enabling us to explore properties of the Kronecker product to accelerate our computations [2], [6]. This is done by choosing the microphone positions in our array and the directions of interest in a separable format.

A 2D separable array has $N_{\text {Mic }}=N_{\text {Mic }} \times N_{\text {MicY }}$ microphones on the positions $(x, y)=\left(x_{i}, y_{j}\right)$, where $i=$
$1, \ldots, N_{\mathrm{MicX}}$ and $j=1, \ldots, N_{\mathrm{Mic}}$. Following the same idea, a $2 \mathrm{D}$ separable grid maps $N_{\text {DirX }} \times N_{\text {DirY }}=N_{\text {Dir }} \mathbf{u}$ directions, defined by the first two entries of $(1),\left(u_{x}, u_{y}\right)=\left(u_{x i}, u_{y j}\right)$, where $i=1, \ldots, N_{\text {DirX }}$ and $j=1, \ldots, N_{\text {DirY }}$.

If both the array and the discrete acoustic grid are separable, our sensing matrix $\mathbf{V}$ can be decomposed as the Kronecker product of two other matrices [1], [6]:

$$
\mathbf{V}=\mathbf{V}_{x} \otimes \mathbf{V}_{y}
$$

The use of separable arrays allows us to use properties of the Kronecker Product to reduce the computational complexity of beamforming and sparse reconstruction algorithms, as shown in [2], [14], with gains of up to $\sqrt{\min \left\{N_{\text {Dir }}, N_{\text {Mic }}\right\}}$ operations.

For the simulations in this article, a deterministic array geometry is used instead of a random geometry as suggested in [9]. A $30 \mathrm{~cm}$ linear non-redundant spacings (minimum missing lags) array [15] is extended to two dimensions as described in [16], [17]. Although we are using an extended 8-microphone non-redundant array, any other linear array geometry, such as uniform linear arrays or a co-prime array [18], can be extended to a $2 \mathrm{D}$ separable array configuration.

We define the directions $\mathbf{u}$ on the discrete grid by uniformly spacing $u_{x}$ and $u_{y}$ between $[-1,1]$, creating a $[33 \times 33]$ separable grid. As a consequence, some $\mathbf{u}$ vectors represent invalid directions, such as $\mathbf{u}=[1,1, \sqrt{-1}]^{T}$, thus estimates from these spurious directions are ignored. The vector of directions of interest $\mathbf{u}$ is uniformly spaced because this distribution yields the minimum coherence for low frequencies and separable arrays, as shown in Section V.

\section{SPARSE RECONSTRUCTION}

We use sparse reconstruction techniques to recover an estimate $\hat{\mathbf{y}}(\omega)$ from equation (6) given the manifold matrix $\mathbf{V}$ (obtained from our system's design) and the measurement vector $\mathbf{x}$.

To assess our array geometry, we look at the coherence of $\mathbf{V}(\omega)$ as a function of the frequency we are reconstructing. The coherence of a matrix $\mathbf{A}$ with normalized columns is an intuitive measure of the correlation between its columns, defined as [19]

$$
\mu(\mathbf{A})=\max _{i \neq j} \mathbf{G}_{i j}, \quad \mathbf{G}=\left|\mathbf{A}^{H} \mathbf{A}\right|,
$$

where $\mathbf{G}_{i j}$ is the element on the $i$ th row and $j$ th column of the Gram matrix G. Note that the columns of $\frac{1}{\sqrt{N_{\text {Mic }}}} \mathbf{V}$ are normalized.

The coherence of a matrix can give us guarantees that our solution is optimally sparse and unique if certain conditions are satisfied. If equation $\mathbf{A x}=\mathbf{b}$ has a solution vector $\mathbf{x}$ such that

$$
\|\mathbf{x}\|_{0}<\frac{1}{2}\left(1+\mu(\mathbf{A})^{-1}\right),
$$

then $\mathrm{x}$ is the unique solution with the minimum number of nonzero elements $\left(\ell_{0}\right.$ norm) [19]-[21]. Therefore, the lower the coherence, the larger the number of nonzero elements that can be uniquely recovered. On the other hand, if the signal is corrupted by noise (thus $\mathbf{A x} \approx \mathbf{b}$ ), some sparse estimation 


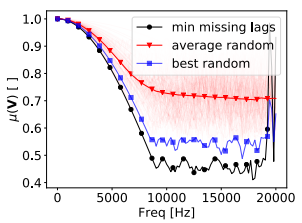

(a)

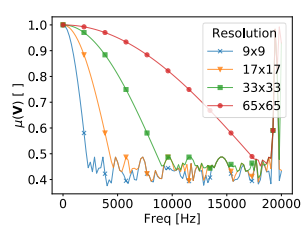

(b)
Figure 1: $\mu(\mathbf{V})$ vs frequency for $30 \mathrm{~cm} 8 \times 8$ microphones minimum missing lags (a) vs. random arrays of similar dimension, and (b) increasing for low frequencies as the resolution increases

algorithms provide theoretical bounds for the reconstruction error that are tighter for lower coherence [22], [23].

For separable arrays, the property [24]

$$
\mu(\mathbf{V})=\mu\left(\mathbf{V}_{x} \otimes \mathbf{V}_{y}\right)=\max \left(\mu\left(\mathbf{V}_{x}\right), \mu\left(\mathbf{V}_{y}\right)\right)
$$

can be used to analyze the coherence of the sensing matrix. This allows us to extrapolate the analysis from [9] to a 2-dimensional system, setting $\mu(\mathbf{V})$ as a parameter for the array performance when using compressive sensing for signal reconstruction or DOA estimation.

Given our chosen array reconstructing a $[33 \times 33]$ discrete uniform field, both described in Section III, the coherence of our sensing matrix as a function of the frequency is seen on Fig. 1a, along with that of several random arrays of similar dimensions. We can see that the non-redundant array has the smallest coherence for almost all the frequency range. From the illustrated data, we can expect this array geometry to perform best when reconstructing signals with main components between $9 \mathrm{kHz}$ and $18 \mathrm{kHz}$.

As we increase the resolution of the reconstructed image, the lower frequency limit of the region of small coherence increases (see eq. (15) in section V), reducing the region with small coherence. This effect is illustrated in Fig. $1 \mathrm{~b}$ and can be explained in the following manner: as we increase resolution the look directions get closer together, turning the neighboring columns of $\mathbf{V}$ more similar and, therefore, increasing its coherence.

\section{Minimal Coherence Space Discretization}

In this section, we prove that a uniformly distributed scan grid yields the minimum coherence for low frequencies and separable array geometry. First, we show this statement for 1D arrays, where $\mathbf{p}=\left[\begin{array}{lll}p & 0 & 0\end{array}\right]^{T}$ and $\mathbf{u}=\left[\begin{array}{lll}u & 0 & \sqrt{1-u^{2}}\end{array}\right]^{T}$, and then we generalize this result for $2 \mathrm{D}$ separable arrays.

Considering the 1D case, define the function $g_{m, n}(\omega)$ as

$$
g_{m, n}(\omega)=\frac{1}{N_{\text {Mic }}^{2}}\left|\mathbf{v}\left(\omega: \mathbf{u}_{m}\right)^{H} \mathbf{v}\left(\omega: \mathbf{u}_{n}\right)\right|^{2}, \quad m \neq n
$$

and set $g_{m, n}=0$ if $m=n$. This way, the coherence is $\mu(\mathbf{V})=\max _{m, n} \sqrt{g_{m, n}(\omega)}$. Substituting $\left[\mathbf{v}\left(\omega: \mathbf{u}_{i}\right)\right]_{k}=$ $e^{-j \frac{\omega}{c} u_{i} p_{k}}$ in (11), we have

$$
g_{m, n}(\omega)=\frac{1}{N_{\text {Mic }}^{2}} \sum_{k=0}^{N_{\text {Mic }}-1} \sum_{\ell=0}^{N_{\text {Mic }}-1} e^{-j \frac{\omega}{c}\left(u_{m}-u_{n}\right)\left(p_{k}-p_{\ell}\right)} .
$$

Note that $g_{m, n}(0)=1$ for all $m, n$, therefore the worstcase coherence will occur for low frequencies. For each term $e^{-j \frac{\omega}{c}\left(u_{m}-u_{n}\right)\left(p_{k}-p_{\ell}\right)}$ in the sum with $k \neq \ell$, there is a single term $e^{-j \frac{\omega}{c}\left(u_{m}-u_{n}\right)\left(p_{\ell}-p_{k}\right)}$, which is the conjugate of the first term. Since the sum of these two terms is $2 \cos \left(\frac{\omega}{c}\left(u_{m}-u_{n}\right)\left(p_{k}-p_{\ell}\right)\right)$ and the sum of the terms with $k=\ell$ is $\frac{1}{N_{\text {Mic }}},(12)$ can be rewritten as

$$
\begin{aligned}
& g_{m, n}(\omega)=\frac{1}{N_{\text {Mic }}}+ \\
& \frac{2}{N_{\text {Mic }}^{2}} \sum_{k=0}^{N_{\text {Mic }}-1} \sum_{\ell=k+1}^{N_{\text {Mic }}-1} \cos \left(\frac{\omega}{c}\left(u_{m}-u_{n}\right)\left(p_{k}-p_{\ell}\right)\right) .
\end{aligned}
$$

Consider that $\omega$ is small enough such that

$\mu(\mathbf{V})^{2}=g_{m^{*}, n^{*}}(\omega), \quad\left(m^{*}, n^{*}\right)=\arg \min _{m, n}\left|u_{m}-u_{n}\right|$.

Equation (14) is true if $\omega$ is very small so that each cosine being summed in (13) is a decreasing function with respect to $\left|u_{m}-u_{n}\right|$, as in this case $g_{m, n}(\omega)$ would be maximized by picking $m$ and $n$ such that $\left|u_{m}-u_{n}\right|$ is minimum. Since $\mu(\mathbf{V})$ is a continuous function of $\omega,(14)$ remains true until $\omega$ is high enough so that $g_{m, n}(\omega)=g_{m^{*}, n^{*}}(\omega)$ for some $m \neq m^{*}$ and $n \neq n^{*}$. This way, $\mu(\mathbf{V})^{2}=g_{m^{*}, n^{*}}(\omega)$ if $0<\omega \leq \omega^{*}$, where

$$
\omega^{*}=\min _{\omega>0}\left\{\omega: \mu(\mathbf{V})=g_{m^{*}, n^{*}}(\omega)\right\} .
$$

Our objective is to obtain the best $\left(u_{0}, u_{1}, \cdots, u_{N_{\text {Dir }}-1}\right)$ such that the coherence $\mu(\mathbf{V})$ is minimum, which is achieved by minimizing $\mu(\mathbf{V})^{2}$. Defining $\omega_{1}>0$ as the smallest critical point of $g_{m^{*}, n^{*}}(\omega), \mu(\mathbf{V})^{2}$ is a decreasing function with respect to $\Delta u^{*}$ for $\omega<\min \left\{\omega^{*}, \omega_{1}\right\}$, so $\Delta u^{*}$ must be maximized. In other words, the set $\left\{u_{i}\right\}_{i=0}^{N_{\text {Dir }}-1}$ that yields the lowest coherence can be obtained by solving the following constrained optimization problem:

$$
\begin{array}{r}
\left(u_{0}^{*}, \cdots, u_{N_{\text {Dir }}-1}^{*}\right)=\arg \max _{u_{0}, \cdots, u_{N_{\text {Dir }}-1}} \min _{i}\left\{u_{i}-u_{i-1}\right\} \\
\text { s.t. }-1 \leq u_{0} \leq u_{1} \leq \cdots \leq u_{N_{\text {Dir }}-1} \leq 1
\end{array}
$$

Although this may seem a difficult problem, its solution is straightforward: (16) must satisfy $u_{i}^{*}-u_{i-1}^{*}=$ constant $\forall i$ because if it were not constant, $u_{k}^{*}-u_{k-1}^{*}=\min _{i} u_{i}^{*}-u_{i-1}^{*}$ could be increased by slightly increasing the value of $u_{k}^{*}-u_{k-1}^{*}$ such that $k$ remains the index that minimizes $u_{i}^{*}-u_{i-1}^{*}$, thus $u_{k}^{*}-u_{k-1}^{*}$ would not maximize the expression. Furthermore, in order to maximize the minimum interval between $u_{i}$ and $u_{i-1}$, the distance between the highest and the lowest element of $\left\{u_{i}\right\}_{i=0}^{N_{\text {Dir }}-1}$ must be as high as possible. As each $u_{i}$ lies in the interval $[-1,1]$, we must have $u_{0}^{*}=-1$ and $u_{N_{\text {Dir }}-1}^{*}=1$. 
Consequently, the solution for (16) is linearly spaced between -1 and +1 :

$$
u_{i}^{*}=-1+\frac{2}{N_{\text {Dir }}-1} k, \quad 0 \leq k \leq N_{\text {Dir }}-1 .
$$

Therefore, the minimal coherence space discretization is the uniform distribution if $0<\omega<\min \left\{\omega^{*}, \omega_{1}\right\}$, where $\omega^{*}$ is given by (15) and $\omega_{1}$ is the smallest frequency such that $\frac{\mathrm{d}}{\mathrm{d} \omega} g_{m^{*}, n^{*}}(\omega)=0$. Even though $\omega^{*}$ has no closedform expression, it can be calculated numerically using (15). For instance, using our non-redundant array with $N_{\text {Mic }}=8$, $N_{\text {Dir }}=33$ and $c=343 \mathrm{~m} / \mathrm{s}$, we have $\omega_{1}=2 \pi \times 12.64 \mathrm{krad} / \mathrm{s}$ and $\omega^{*}=2 \pi \times 9.17 \mathrm{krad} / \mathrm{s}$, which agrees with Fig. 1a.

For two-dimensional separable arrays, $\mathbf{u}=$ $\left[\begin{array}{lll}u_{x} & u_{y} & \sqrt{1-u_{x}^{2}-u_{y}^{2}}\end{array}\right]^{T}$ and $\mu(\mathbf{V})=\max \left\{\mu\left(\mathbf{V}_{x}\right), \mu\left(\mathbf{V}_{y}\right)\right\}$. The minimal value for $\mu(\mathbf{V})$ can only be achieved when both $\mu\left(\mathbf{V}_{x}\right)$ and $\mu\left(\mathbf{V}_{y}\right)$ are minimized, which happens when both $u_{x}$ and $u_{y}$ follow the uniform distribution (17).

\section{DOA ESTIMATION}

In this section, we verify our results using a separable array to estimate the direction of arrival (DOA) of a sinusoidal source at a random location with a sinusoidal interference fixed at the central position $(0,0)$, and measurement noise with SNR of $30 \mathrm{~dB}$, with sampling frequency of $64 \mathrm{kHz}$ and FFTs with $L=100$ points using a rectangular window with 100 points. The DOA of the source of interest is obtained using a single snapshot by Delay-and-Sum (DAS) beamformers and by sparse estimation using Orthogonal Matching Pursuit (OMP) [25], [26]. We also include results obtained using Regularized Minimum Variance Distortionless Response (Regularized MVDR) beamformers, designed with the exact noise covariance matrix (used as a benchmark). In practice MVDR beamformers need to estimate the noise covariance matrix, so they are not applicable to the single snapshot case. Since MUSIC [27] also needs multiple snapshots, we do not include it in our simulations. The simulations assume both the source and the interference to be sinusoids with the same magnitude and frequency $f_{0}$ and random phase. All three algorithms search a grid with $33 \times 33$ equally-spaced directions. To determine the precision of a single reconstruction, we assume previous knowledge of the number of sources present. The two largest values on the reconstructed image are considered the located sources, and the angle between the source of interest and the nearest of the two located sources is taken as the location error.

The DAS beamformer requires no configuration parameters, and its implementation uses the separability of the matrix $\mathbf{V}$ trough the Kronecker Product (eq. (7)) to reduce its computational complexity. The regularized MVDR is a variation on the MVDR beamformer initially proposed in [13], with an added constant in the main diagonal of the noise covariance matrix to provide robustness (diagonal loading) [28]. The diagonal regularization added is of value $10^{-2}$. MVDR beamforming requires previous knowledge of the noise covariance matrix, and in this article the OMP algorithm is not fed this information. The OMP algorithm was set for $1 \%$ maximum sparsity

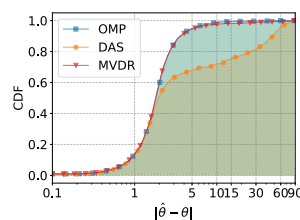

(a)

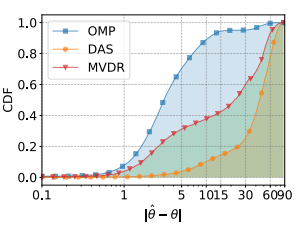

(b)
Figure 2: DOA error cumulative distribution for different algorithms at (a) $16 \mathrm{kHz}$ and (b) $2 \mathrm{kHz}$

and $10^{-3}$ error stop condition. It also uses the separability of the matrix $\mathbf{V}$ through the Kronecker product to accelerate its computations, as described in [6], [14].

After $6 \times 10^{3}$ realizations of the DOA experiment with random directions, we calculate the DOA error distribution for each algorithm. Fig. 2a represents the cumulative distribution function of the angular error $|\hat{\theta}-\theta|$ for $f_{0}=16 \mathrm{kHz}$, frequency in the low coherence region for the array, and Fig. $2 \mathrm{~b}$ is for $f_{0}=2 \mathrm{kHz}$, a frequency with a higher coherence (see Fig. 1a). From these results, we expect smaller deviations from the ideal DOA when using the OMP, even in low frequencies with high coherence.

It is worth noting that, in the exceptional case where all signal directions are in the reconstruction grid, since MVDR has full noise covariance information, it presents zero DOA error for all in-grid directions.

\section{SignAL RECOVERY}

Our goal with this simulation is to recover an arbitrary signal coming from a specific direction, while there is a second signal source in the field that will be treated as noise. The signal estimation is obtained by recovering $\mathbf{x}(\omega)$ estimates for all $\omega$ in our Discrete Fourier Transform, and performing the inverse transform to obtain the signal in the time domain.

For the signal recovery, STFT is used with the Hanning window with $50 \%$ overlap as recommended in [29], [30]. We also pad our signal with zeros at the end of the window, doubling its size, as [31] suggests to mitigate time aliasing effects. We compare the reconstruction results obtained by using the compressive sensing algorithm OMP with those obtained with DAS and regularized MVDR beamformers. The parameter settings for the algorithms are the same as discussed on section VI.

The simulations were run reconstructing a $[33 \times 33]$ field, with the desired signal coming from the central direction $\mathbf{u}_{s}=\left[\begin{array}{lll}0 & 0 & 1\end{array}\right]^{T}$, first with the signal being a filtered white noise with $11 \mathrm{kHz}-17 \mathrm{kHz}$ bandwidth, to operate within the best coherence margin of the array, as seen on Fig. 1a, and a second case where the signal bandwidth is $2 \mathrm{kHz}-17 \mathrm{kHz}$, outside the low coherence region. The interference signal comes from the direction $\mathbf{u}_{n}=\left[0 \frac{-1}{2} \frac{\sqrt{3}}{2}\right]^{T}$, its signal being a filtered white noise with $0-18 \mathrm{kHz}$ bandwidth. Independent Gaussian noise is added to each microphone to create measurement noise with 


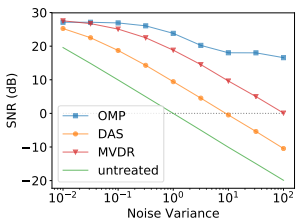

(a)

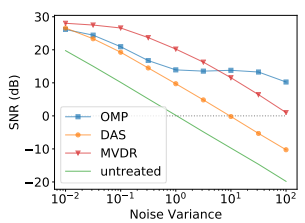

(b)
Figure 3: Reconstruction SNR vs interference variance for (a) $11 \mathrm{kHz}-17 \mathrm{kHz}$ signal and (b) $2 \mathrm{kHz}-17 \mathrm{kHz}$

30 dB Signal-to-Noise Ratio and 150 realizations of each case were performed.

The SNR for the recovered signals are illustrated in Fig. 3, where untreated represents the SNR for the direct signal captured by a microphone located at position $[0,0]$.

\section{CONCLUSION}

Compressive sensing was shown in [9] to be an effective solution for the one-dimensional source localization problem when working with sensor arrays. We extended this result to 2D source localization. We also show that compressive sensing techniques can be used to reconstruct the signal coming from a specific direction, similar to a super-directional microphone.

For both applications we show that more precise results than traditional beamformers can be expected from the OMP method, without the prior knowledge that MVDR requires. There are still topics to be explored further, such as carrying over information between frequency bins when working with wideband reconstruction, as [12], and using DOA knowledge as a starting point for the support in the sparse reconstruction.

\section{REFERENCES}

[1] F. P. Ribeiro and V. H. Nascimento, "Fast transforms for acoustic imaging - part I: Theory," IEEE Transactions on Image Processing, vol. 20, no. 8, pp. 2229-2240, 2011

[2] — , "Fast transforms for acoustic imaging - part II: Applications," IEEE Transactions on Image Processing, vol. 20, no. 8, pp. 2241-2247, 2011

[3] H. L. Van Trees, Optimum Array Processing: Part IV of Detection, Estimation, and Modulation Theory. New York, NY: John Wiley \& Sons, 2002 .

[4] T. F. Brooks and W. M. Humphreys, "A deconvolution approach for the mapping of acoustic sources (DAMAS) determined from phased microphone arrays," Journal of Sound and Vibration, vol. 294, no. 4, microphone arrays,"
pp. 856-879, 2006.

[5] T. Yardibi, J. Li, P. Stoica, N. S. Zawodny, and L. N. C. Iii, "A covariance fitting approach for correlated acoustic source mapping," The Journal of the Acoustical Society of America, vol. 127, no. 5, pp. 2920-2931, May 2010, 00014

[6] B. Masiero and V. H. Nascimento, "Revisiting the Kronecker array transform," IEEE Signal Processing Letters, vol. 24, no. 5, pp. 525529, May 2017.

[7] A. C. Gurbuz, J. H. McClellan, and V. Cevher, "A compressive beamforming method," in Proc. Speech and Signal Processing 2008 IEEE Int. Conf. Acoustics, Mar. 2008, pp. 2617-2620.

[8] G. F. Edelmann and C. F. Gaumond, "Beamforming using compressive sensing," The Journal of the Acoustical Society of America, vol. 130, no. 4, pp. EL232-EL237, 2011.
[9] A. Xenaki, P. Gerstoft, and K. Mosegaard, "Compressive beamforming," The Journal of the Acoustical Society of America, vol. 136, p. 260, 07 The Jo
2014.
C. . .

[10] C. F. Gaumond and G. F. Edelmann, "Sparse array design using statistical restricted isometry property," The Journal of the Acoustical Society of America, vol. 134, no. 2, pp. EL191-EL197, 2013.

[11] A. Xenaki and P. Gerstoft, "Grid-free compressive beamforming," The A. Xenaki and P. Gerstoft, "Grid-free compressive beamforming," The
Journal of the Acoustical Society of America, vol. 137, no. 4, pp. 1923-
1935,2015 .

[12] L. Xu, K. Zhao, J. Li, and P. Stoica, "Wideband source localization using sparse learning via iterative minimization," Signal Processing, vol. 93, no. 12 , pp. 3504-3514, 2013

[13] J. Capon, "High-resolution frequency-wavenumber spectrum analysis," Proceedings of the IEEE, vol. 57, no. 8, pp. 1408-1418, Aug 1969.

[14] V. H. Nascimento, M. C. Silva, and B. S. Masiero, "Acoustic image estimation using fast transforms," in Sensor Array and Multichannel Signal Processing Workshop (SAM), 2016 IEEE. IEEE, 2016, pp. 1-5.

15] E. Vertatschitsch and S. Haykin, "Nonredundant arrays," Proceedings of the IEEE, vol. 74, no. 1, pp. 217-217, Jan 1986

[16] F. P. RIBEIRO, "Arrays de microfones para medida de campos acusticos," Ph.D. dissertation, Escola Politecnica, Universidade de Sao Paulo, 2012

17] B. S. Masiero, C. S. B. Arroyo, and V. H. Nascimento, "Fast acoustic imaging with separable arrays," in Proceedings, 2015 International Symposium on Solid Mechanics, Belo Horizonte, MG, Brazil, May 2015, pp. $1-15$

[18] P. P. Vaidyanathan and P. Pal, "Sparse sensing with co-prime samplers and arrays," IEEE Transactions on Signal Processing, vol. 59, no. 2, pp. 573-586, Feb 2011

19] M. Elad, Sparse and redundant representations: from theory to applications in signal and image processing. New York: Springer, 2010, oCLC: ocn646114450.

20] D. L. Donoho and M. Elad, "Optimally sparse representation in general (nonorthogonal) dictionaries via 11 minimization," Proceedings of the National Academy of Sciences, vol. 100, no. 5, pp. 2197-2202, 2003 [Online]. Available: http://www.pnas.org/content/100/5/2197

21] R. Gribonval and M. Nielsen, "Sparse representations in unions of bases," IEEE Transactions on Information Theory, vol. 49, no. 12, pp. $3320-3325$, Dec 2003.

22] M. F. Duarte and Y. C. Eldar, "Structured compressed sensing: Fron theory to applications," IEEE Transactions on Signal Processing, vol. 59 no. 9, pp. 4053-4085, Sep. 2011

[23] D. L. Donoho, M. Elad, and V. N. Temlyakov, "Stable recovery of sparse overcomplete representations in the presence of noise," IEE Transactions on Information Theory, vol. 52, no. 1, pp. 6-18, Jan 2006

[24] S. Jokar, "Sparse recovery and kronecker products," in 2010 44th Annuс Conference on Information Sciences and Systems (CISS), March 2010, pp. $1-4$.

[25] Y. C. Pati, R. Rezaiifar, and P. S. Krishnaprasad, "Orthogonal matching pursuit: recursive function approximation with applications to wavele decomposition," in Proceedings of 27th Asilomar Conference on Signals, Systems and Computers, Nov 1993, pp. 40-44 vol.1.

[26] J. A. Tropp and A. C. Gilbert, "Signal recovery from random measurements via orthogonal matching pursuit," IEEE Transactions on Information Theory, vol. 53, no. 12, pp. 4655-4666, Dec 2007.

[27] R. Schmidt, "Multiple emitter location and signal parameter estimation," IEEE Transactions on Antennas and Propagation, vol. 34, no. 3, pp. 276-280, March 1986

[28] J. Li, P. Stoica, and Z. Wang, "On robust capon beamforming and diagonal loading," in 2003 IEEE International Conference on Acoustics, Speech, and Signal Processing, 2003. Proceedings. (ICASSP '03)., vol. 5, April 2003, pp. V-337.

29] S. Boll, "Suppression of acoustic noise in speech using spectral subtraction," IEEE Transactions on Acoustics, Speech, and Signal Processing, vol. 27, no. 2, pp. 113-120, April 1979 .

[30] D. Griffin, "Signal estimation from modified short-time Fourier transform," and Signal Processing IEEE Transactions on Acoustics, Speech, vol. 32, no. 2, pp. 236-243, Apr. 1984

[31] J. Allen, "Short term spectral analysis, synthesis, and modification by discrete fourier transform," IEEE Transactions on Acoustics, Speech, and Signal Processing, vol. 25, no. 3, pp. 235-238, June 1977. 